\title{
DYNAMICS OF A SUSPENSION OF SPHERES AND RIGID POLYMERS: EFFECT OF GEOMETRICAL MISMATCH
}

\author{
Radu P. Mondescu and M. Muthukumar \\ Department of Physics and Astronomy, and Polymer Science $\& 6$ Engineering Department and Materials Research Science and \\ Engineering Center \\ University of Massachusetts, Amherst, MA 01003
}

(February 21, 2018)

\begin{abstract}
An effective medium approach together with a multiple scattering formalism is considered to study the steady-state dynamics of suspensions of spheres and rigid stiff polymer chains without excluded volume interactions. The polymer chains are taken to be so long that Gaussian statistics is applicable. We have considered the dynamics of probe objects in a solution containing spheres and polymers. The probe object is either a sphere or a polymer. We have studied different conditions of the solution where some or all of the spheres are frozen in space. The effective medium equations have been solved self-consistently for finite volume fractions of spheres $\Phi^{S P}$ and polymers $\Phi^{P O L}$, respectively, and the important dimensionless variables that are controlling the dynamical behavior have been identified. In particular, the role of the geometrical parameter $t=\frac{R_{g}}{a}$ ( $a$ is the radius of any sphere and $R_{g}$ the radius of gyration of a polymer chain) is discussed. The translational diffusion coefficients of the moving probe sphere $D^{S}$ and of the probe polymer chain $D^{P}$, and the shear viscosity of the suspensions have been derived. When the polymers are present in the solution,both the friction coefficient of the labeled mobile sphere or that of the probe polymer chain and the shear viscosity diverge as $\Phi^{P O L} \rightarrow 0.31$. Also, when polymers are diffusing in a suspension of fixed spheres an optimum range of $\Phi^{S P}$ that maximizes the difference in the diffusion coefficients of polymer chains characterized by distinct $t$ values has been noticed.
\end{abstract}

\section{INTRODUCTION}

Starting with the pioneering work of Einstein [1], a lot of effort was devoted to developing the hydrodynamics of suspensions at finite concentrations, owing to both the intrinsic challenge of the problem and to the considerable utility of such a theory in the rheology of concentrated polymer suspensions. Most of the work done is related to the hydrodynamics of suspensions of hard spheres, specifically to the computation of virial-type expansions - in the volume fraction $\Phi$ (low but non-zero) of the suspended particles - for the change in viscosity $\delta \eta$ and in the friction coefficients $\zeta$ (translational and rotational). An incomplete review of the development of this field could include the divergence-free theories of Peterson and Fixman [2] and Batchelor and Green [3], the multiple scattering method elaborated by Freed and Muthukumar [4,5] and Muthukumar and Freed [6, 4], the expansion in correlation functions used by Beenaker [8] and the angular momentum diagrammatic expansion combined with the multiple scattering technique in Thomas and Muthukumar [9].

The viscosity and frictional properties of infinitely diluted polymer solutions received a wide interest (see Ref. [10]), but few attempts were made to construct a detailed theory for finite concentrations $11-13$, and even less theoretical results are available for dispersions of distinct types of elements.

In this paper we derive explicit expressions for the change in viscosity due to suspended objects and for the translational diffusion coefficients, as functions of the solid filling fractions of spheres and of polymer chains, $\Phi^{S P}$ and $\Phi^{P O L}$, respectively, for a suspension of polymers and rigid, fixed spheres, in the stationary, timeindependent limit. Qualitative features of the hydrodynamic behavior of the mixed suspensions are discussed and it is shown that when the polymers are present in the solution, the dynamics is frozen at $\Phi^{P O L} \simeq 0.31$. Results in the limits of very low 14 and very high polymer concentrations (Rouse) and also for the dynamics of a random array of spheres 15, are recovered. We have found that the interaction between polymers, spheres and the fluid is controlled by the dimensionless parameters $\beta=\frac{R_{g}}{\xi}, x=\frac{a}{\xi}$ and $t=\frac{R_{g}}{a}$, where $\xi=\xi\left(\Phi^{S P}, \Phi^{P O L}, t\right)$ is the hydrodynamic screening length.

Technically, our calculations are based on the multiple scattering formalism [4, 6, 15,5] and on the assumption that the physical properties of the suspension, averaged over the random position distribution of the particles, are equivalent to those of an homogeneous effective fluid characterized by the effective viscosity $\eta^{\text {eff }}$ and by the altered Oseen propagator of the velocity field $\mathcal{G}\left(\mathbf{r}, \mathbf{r}^{\prime}\right)$ (to be defined later).

The main simplifying hypothesis is the absence of 
excluded volume interactions - sphere-sphere,polymerpolymer,or polymer-sphere-both the polymer chains and the spheres being penetrable,ghost-type objects. Also, the solvent is assumed incompressible and described by a linearized Navier-Stokes (N-S) equation.

Other assumptions made and the basic features of the theory are :

a) the suspension of spheres and polymers is monodisperse, all spheres having the radius $a$ and all polymers having the length $L$.

b) except the sphere producing the flow, all others are fixed. All polymers are Gaussian.

c) the specific calculations are valid only in the limit of steady-state shear flow in the linear response regime, when the average force $\langle\mathbf{F}(\mathbf{r})\rangle$ exerted upon the fluid is given by :

$$
\langle\mathbf{F}(\mathbf{r})\rangle=(\mathrm{K} *\langle\mathbf{v}\rangle)(\mathbf{r})
$$

where $\mathrm{K}$ is a tensor-like quantity and $*$ is the convolution operator: $\mathrm{f} * \mathrm{~g}=\int f\left(\mathbf{r}-\mathbf{r}^{\prime}\right) g\left(\mathbf{r}^{\prime}\right) d \mathbf{r}^{\prime}$.

d) both the spheres and the polymer chains are randomly distributed, and after the configurational average is taken the suspension becomes isotropic.

e) we employ the usual preaveraging approximation [10], where one replaces the value of the effective Oseen tensor $\mathcal{G}\left(\mathbf{r}, \mathbf{r}^{\prime}\right)$ and of its generalized inverse with their configurational averages $\left\langle\mathcal{G}\left(\mathbf{r}, \mathbf{r}^{\prime}\right)\right\rangle$ and $\left\langle\mathcal{G}^{-1}\left(\mathbf{r}, \mathbf{r}^{\prime}\right)\right\rangle$.

f) two models have been analyzed :

S-P an ensemble of rigid spheres in a dispersion of polymers. All but one sphere are fixed. The tracer sphere generates the flow.

P-S a collection of polymer chains immersed in a suspension of immobile spheres, with an external flow imposed.

The hydrodynamic interaction between fluid and particles is implemented by enforcing stick boundary conditions. In each example we calculate the translational diffusion coefficient of the test object ( a polymer chain or the tracer sphere ) and the change in the shear viscosity of the solution due to the presence of the added particles, as functions of $\Phi^{S P}, \Phi^{P O L}$ and the coupling parameter $t=\frac{R_{g}}{a}$.

The paper is organized as follows : next section deals with the formal theory of the steady-state velocity flow in a solution of $\mathrm{N}_{P}$ polymer chains and $\mathrm{N}_{S}$ fixed spheres, in Section III we apply the derivation to the separate cases of polymers and spheres, which are needed in Sec. IV] where we solve and show the results for the four specific examples mentioned previously. Finally, in Section \, we present, discuss, and review the main findings of our paper. For the reader interested in reproducing and extending our results, detailed calculations are displayed in the Appendices.

\section{FORMAL THEORY OF THE VELOCITY FIELD IN A SUSPENSION OF POLYMERS AND SPHERES}

To describe the dynamics of a hydrodynamic system, a knowledge of the velocity field $\mathbf{v}(\mathbf{r}, \mathrm{t})$ produced by the forces acting upon the fluid is required. Our goal is to find an expression for $\mathbf{v}(\mathbf{r})$ (stationary limit) for the system we are interested in and to extract the appropriate physical information (diffusion coefficient, viscosity). This is accomplished using the multiple scattering technique as shown below.

\section{A. Fluid and Particles}

Under the assumptions indicated in the previous section and taking the fluid density equal to unity, the stationary velocity field for an incompressible, newtonian viscous fluid is governed by the linearized N-S equation :

$$
-\eta_{0} \triangle \mathbf{v}(\mathbf{r})+\nabla p(\mathbf{r})=\mathbf{F}(\mathbf{r})
$$

where $\mathbf{F}(\mathbf{r}, \mathrm{t})$ and $p(\mathbf{r}, t)$ are the external force driving the flow ( including the random thermal contribution ) and the pressure, and $\eta_{0}$ is the kinematic shear viscosity coefficient. Introducing the Fourier transform of any function $\mathbf{A}(\mathbf{r})$ as :

$$
\begin{aligned}
& \mathbf{A}(\mathbf{r})=\int \frac{\mathrm{d} \mathbf{k}}{(2 \pi)^{3}} \mathbf{A}(\mathbf{k}) \exp (-i \mathbf{k} \cdot \mathbf{r}) \equiv \int_{\mathbf{k}} \mathbf{A}(\mathbf{k}) \exp (-i \mathbf{k} \cdot \mathbf{r}) \\
& \mathbf{A}(\mathbf{k})=\int \mathrm{d} \mathbf{r} \mathbf{A}(\mathbf{r}) \exp (i \mathbf{k} \cdot \mathbf{r})
\end{aligned}
$$

one finds the formal solution of Eq. (11) as a convolution :

$$
\mathbf{v}(\mathbf{r})=(\mathbf{G} * \mathbf{F})(\mathbf{r})
$$

with $\mathbf{G}\left(\mathbf{r}, \mathbf{r}^{\prime}\right)$ being the Oseen tensor [16] (in dyadic notation) :

$$
\begin{gathered}
\mathbf{G}(\mathbf{k})=\frac{\mathbb{1}-\hat{\mathbf{k}} \hat{\mathbf{k}}}{\eta_{0} k^{2}} \\
\mathbf{G}\left(\mathbf{r}, \mathbf{r}^{\prime}\right)=\frac{1}{8 \pi \eta_{0}\left|\mathbf{r}-\mathbf{r}^{\prime}\right|}\left(\mathbb{1}+\frac{\left(\mathbf{r}-\mathbf{r}^{\prime}\right)\left(\mathbf{r}-\mathbf{r}^{\prime}\right)}{\left|\mathbf{r}-\mathbf{r}^{\prime}\right|^{2}}\right)
\end{gathered}
$$

Here, $\mathbb{1}$ is the unit tensor and $\hat{\mathbf{k}}$ is the unit vector pointing in the $\mathbf{k}$ direction.

Physically, $\mathbf{G}\left(\mathbf{r}, \mathbf{r}^{\prime}\right)$ - the force propagator - is conveying the hydrodynamic disturbances, converting a pointlike force acting at $\mathbf{r}^{\prime}$ to the velocity of a fluid particle located at $\mathbf{r}$. When a fixed object is introduced in the 
fluid, the velocity flow is perturbed due to the scattering off the particle surface. One needs then to include in Eq. (1) a term $\mathbf{F}^{O B J \rightarrow F L}(\mathbf{r})$ corresponding to the force exerted by the particle upon the fluid :

$$
-\eta_{0} \triangle \mathbf{v}(\mathbf{r})+\nabla p(\mathbf{r})=\mathbf{F}(\mathbf{r})+\mathbf{F}^{O B J \rightarrow F L}(\mathbf{r})
$$

To couple in a simple way the dynamics of the solvent and of the object, we choose no-slip boundary conditions :

$$
\mathbf{v}^{\mathrm{FLUID}}\left(\mathbf{r}_{s}\right)=\mathbf{v}^{\mathrm{OBJ}}\left(\mathbf{r}_{s}\right), \quad \mathbf{r}_{s} \in \text { object surface. }
$$

In Eq. (5) $\mathbf{F}(\mathbf{r})$ is zero inside and on the surface of the particles and $\mathbf{F}^{O B J \rightarrow F L}(\mathbf{r})$ is non-zero only on the surface of the suspended particles. The equation of motion is valid throughout the volume of the fluid. Because we want to replace the fluid and the suspended particles with an effective medium characterized by an average velocity field defined everywhere, it is necessary to apply Eq. (5) inside the volume of the particles as well. This is true provided the full stress tensor $\Pi(\mathbf{r})$ [17, 4 ] obeys ( neglecting the inertial term in the equation of motion of the object ) :

$$
\begin{aligned}
& \nabla \cdot \Pi(\mathbf{r})=0 \quad, \mathbf{r} \in \text { inside object. } \\
& \nabla \cdot \Pi\left(\mathbf{r}_{s}\right)=\mathbf{F}^{O B J \rightarrow F L}\left(\mathbf{r}_{s}\right), \mathbf{r}_{s} \in \text { object surface. }
\end{aligned}
$$

The formal solution of Eq. (5) can be written as :

$$
\mathbf{v}(\mathbf{r})=\left.\mathbf{G} * \mathbf{F}\right|_{\mathbf{r}}+\left.\mathbf{G} * \mathbf{F}^{O B J \rightarrow F L}\right|_{\mathbf{r}}
$$

Using the boundary condition (6), we can eliminate the unknown quantity $\mathbf{F}^{\mathrm{O} B J \rightarrow F L}(\mathbf{r})$ - as shown explicitly in the next section - and arrive at the exact equation that gives the microscopic velocity field everywhere in the fluid :

$$
\mathbf{v}(\mathbf{r})=\left.\mathbf{G} * \mathbf{F}\right|_{\mathbf{r}}+\left.\mathbf{G} * \mathbf{T}^{O B J} * \mathbf{G} * \mathbf{F}\right|_{\mathbf{r}}
$$

where the multiple convolution product means :

$$
\left.\mathbf{f} * \mathbf{g} * \mathbf{h}\right|_{\mathbf{r}}=\int d \mathbf{r} d \mathbf{r}^{\prime} \mathbf{f}\left(\mathbf{r}-\mathbf{r}^{\prime}\right) \cdot \mathbf{g}\left(\mathbf{r}^{\prime}-\mathbf{r}^{\prime \prime}\right) \cdot \mathbf{h}\left(\mathbf{r}^{\prime \prime}\right)
$$

and $\mathbf{T}^{O B J}$ represents the flow propagator, a tensor operator that transforms the velocity field incident on the object in a force field located on its surface and acting upon the fluid. $\mathbf{T}^{O B J}$ depends in general on the position, structure and geometry of the object. The interpretation of (9) is straightforward : there are two contributions to the velocity of the fluid $\mathbf{v}(\mathbf{r})$ at point $\mathbf{r}$, a direct wave due to the external force $\mathbf{F}$ acting at some point $\mathbf{r}^{\prime}$, and an indirect contribution coming from the scattering off the surface of the object of the disturbance produced by $\mathbf{F}$ at $\mathbf{r}^{\prime}$ (see Fig. 1).

When many objects are present in the solution, one should add all the contributions coming from all possible scattering sequences but, even for two bodies only, Eq. (9) becomes a series with an infinite number of terms, the convergence of which might be problematic. For some specific cases (polymers, spheres) [12, 15], these difficulties can be overcome, which was the reason we decided to investigate solutions of mixed polymer chains and spheres. For example, when two objects labeled a and $b$ are present in the solution, the multiple scattering expression for the velocity field $\mathbf{v}(\mathbf{r})$ will be :

$$
\begin{aligned}
\mathbf{v}(\mathbf{r})= & \left.\mathbf{G} * \mathbf{F}\right|_{\mathbf{r}}+\left.\mathbf{G} *\left\{\mathbf{T}_{a}+\mathbf{T}_{b}\right\} * \mathbf{G} * \mathbf{F}\right|_{\mathbf{r}} \\
& +\left.\mathbf{G} *\left\{\mathbf{T}_{a} * \mathbf{G} * \mathbf{T}_{b}+\mathbf{T}_{b} * \mathbf{G} * \mathbf{T}_{a}\right\} * \mathbf{G} * \mathbf{F}\right|_{\mathbf{r}} \\
& +\mathbf{G} *\left\{\mathbf{T}_{a} * \mathbf{G} * \mathbf{T}_{b} * \mathbf{G} * \mathbf{T}_{a}\right. \\
& \left.+\mathbf{T}_{b} * \mathbf{G} * \mathbf{T}_{a} * \mathbf{G} * \mathbf{T}_{b}\right\}\left.* \mathbf{G} * \mathbf{F}\right|_{\mathbf{r}}+\ldots
\end{aligned}
$$

Being interested only in the average properties of the system, we will average in Eq. (9) upon the random position of the body, thus obtaining the average velocity field $\mathbf{u}(\mathbf{r})$ :

$$
\begin{aligned}
\mathbf{u}(\mathbf{r})=\langle\mathbf{v}(\mathbf{r})\rangle= & \frac{1}{V} \int d \mathbf{R}^{0} \mathbf{v}(\mathbf{r})=\left.\mathbf{G} * \mathbf{F}\right|_{\mathbf{r}} \\
& +\left.\mathbf{G} *\left\langle\mathbf{T}^{O B J}\right\rangle * \mathbf{G} * \mathbf{F}\right|_{\mathbf{r}}
\end{aligned}
$$

with $\mathbf{R}^{0}$ denoting the position of the center of mass of the object. Note that if excluded volume interactions are considered, appropriate particle distribution functions should be used.

To make the connection with the experimentally measurable quantities, we will turn to Eq. (5) and average it directly over the particle distribution to get the NavierStokes equation describing the effective fluid :

$$
-\eta_{0} \triangle \mathbf{u}(\mathbf{r})+\nabla\langle p(\mathbf{r})\rangle-\left.\boldsymbol{\Sigma} * \mathbf{u}\right|_{\mathbf{r}}=\mathbf{F}(\mathbf{r})
$$

In writing the equation above we introduced a new quantity, the $\boldsymbol{\Sigma}\left(\mathbf{r}, \mathbf{r}^{\prime}\right)$ operator, called the self-energy of the fluid and defined by the relation :

$$
\left.\boldsymbol{\Sigma} * \mathbf{u}\right|_{\mathbf{r}}=\left\langle\mathbf{F}^{O B J \rightarrow F L}(\mathbf{r})\right\rangle
$$

Note that this relation implies a linear response regime of the shear flow.

$\boldsymbol{\Sigma}\left(\mathbf{r}, \mathbf{r}^{\prime}\right)$ is the essential quantity that encompasses all the information regarding the change in the viscoelastic properties of the fluid due to the presence of the object(s). To illustrate this, let us introduce the Fourier transform of the self-energy, knowing that the averaged solution is translationally invariant :

$$
\boldsymbol{\Sigma}(\mathbf{k})=\int d \mathbf{r} \boldsymbol{\Sigma}\left(\mathbf{r}-\mathbf{r}^{\prime}\right) \exp \left(i \mathbf{k} \cdot\left(\mathbf{r}-\mathbf{r}^{\prime}\right)\right)
$$

Then Eq. (9) becomes : 


$$
\left[\eta_{0} k^{2} \mathbb{1}-\boldsymbol{\Sigma}(\mathbf{k})\right] \cdot \mathbf{u}(\mathbf{k})-i \mathbf{k} p(\mathbf{k})=\mathbf{f}(\mathbf{k})
$$

Using the incompressibility condition $\hat{\mathbf{k}} \cdot \mathbf{u}(\mathbf{k})=0$ and decomposing $\boldsymbol{\Sigma}\left(\mathbf{r}, \mathbf{r}^{\prime}\right)$ into its transverse and longitudinal parts applying the relations :

$$
\begin{aligned}
\mathbf{\Sigma}(\mathbf{k}) & =\Sigma_{\perp}(\mathbf{k})(\mathbb{1}-\hat{\mathbf{k}} \hat{\mathbf{k}})+\Sigma_{\|}(\mathbf{k}) \hat{\mathbf{k}} \hat{\mathbf{k}} \\
(\mathbb{1}-\hat{\mathbf{k}} \hat{\mathbf{k}}) \cdot(\mathbb{1}-\hat{\mathbf{k}} \hat{\mathbf{k}}) & =(\mathbb{1}-\hat{\mathbf{k}} \hat{\mathbf{k}}) \\
\hat{\mathbf{k}} \hat{\mathbf{k}} \cdot(\mathbb{1}-\hat{\mathbf{k}} \hat{\mathbf{k}}) & =0
\end{aligned}
$$

we can eliminate the pressure $p(\mathbf{k})$ to solve Eq. (15) for the Fourier component $\mathbf{u}(\mathbf{k})$ of the averaged velocity field $\mathbf{u}(\mathbf{r})$ :

$$
\begin{aligned}
& \mathbf{u}(\mathbf{k})=\mathcal{G}(\mathbf{k}) \cdot \mathbf{F}(\mathbf{k}) \\
& \mathcal{G}(\mathbf{k})=\frac{\mathbb{1}-\hat{\mathbf{k}} \hat{\mathbf{k}}}{\eta_{0} k^{2}-\Sigma_{\perp}(\mathbf{k})}
\end{aligned}
$$

We recover then, immediately :

$$
\mathbf{u}(\mathbf{r})=\left.\mathcal{G} * \mathbf{F}\right|_{\mathbf{r}}
$$

The interpretation of $\boldsymbol{\Sigma}$ emerges clearly. What we achieved is to replace the initial system fluid + object(s) with an effective medium of a certain viscosity $\eta^{\text {eff }}$ where the hydrodynamic disturbances are propagated by the modified Oseen tensor $\mathcal{G}(\mathbf{k})$, with all the complexities of the multiple scattering processes involving the suspended object(s) captured by the $\boldsymbol{\Sigma}$ tensor.

In the hydrodynamic limit $|\mathbf{k}| \rightarrow 0$, the total change in viscosity is given by :

$$
\frac{\eta^{\mathrm{eff}}-\eta_{0}}{\eta_{0}}=-\lim _{\mathbf{k} \rightarrow 0} \frac{1}{\eta_{0}} \frac{\partial}{\partial k^{2}} \Sigma_{\perp}(\mathbf{k})
$$

If $\Sigma_{\perp}(\mathbf{k}=0) \neq 0$, the hydrodynamic interaction is screened, with the screening length $\xi$ given by:

$$
\xi^{-2}=-\frac{1}{\eta_{0}} \Sigma_{\perp}(\mathbf{k}=0)
$$

Similarly, formulas for the friction coefficients $\boldsymbol{\zeta}$ (translational, rotational, cross translational-rotational ) of the object can be derived from $\boldsymbol{\Sigma}(\mathbf{k})$. We will explicitly show this, for the translational friction coefficient, in the following sections.

More practical, from an experimental point of view, is the diffusion coefficient $D$ of a particle moving in a solution, calculated from the Einstein formula:

$$
D(\Phi)=\frac{k_{B} T}{\zeta(\Phi)}
$$

where $\mathrm{T}=$ temperature, $k_{B}$ is the Boltzmann constant and $\Phi$ is the volume fraction of the suspended particles in the solution. Note that in general the friction coefficient is a tensor-like quantity, in which case the previous expression should be adjusted correspondingly. The dimensionless variable that we have computed and plotted in this paper is the relative translational diffusion coefficient :

$$
\frac{D_{t}(\Phi)}{D_{t}^{0}}=\frac{\zeta_{t}^{0}}{\zeta_{t}(\Phi)}
$$

with $D_{t}^{0}$ the translational diffusion coefficient of a particle in the pure fluid (e.g. $D_{t}^{0}=\frac{k_{B} T}{6 \pi \eta_{0} a}$ for a sphere and $D_{t}^{0}=\frac{8 \sqrt{2}}{3} \frac{k_{B} T}{6 \pi \sqrt{2 \pi} \eta_{0} R_{g}}$ for a single polymer chain ). In the remaining of this work, we will denote this relative diffusion coefficient simply by $D$.

Having established that $\boldsymbol{\Sigma}(\mathbf{k})$ contains the relevant dynamical information, we need to actually compute it by relating it to the known quantity $\mathbf{T}^{O B J}$. Here lies the essence of the multiple scattering formalism. Averaging Eq. (8) directly and using the definition of $\boldsymbol{\Sigma}$ from Eq. (13) we can rewrite the solution for the averaged velocity field $\mathbf{u}(\mathbf{r})$ in terms of the $\mathbf{G}$ propagator of the pure fluid :

$$
\mathbf{u}(\mathbf{r})=\left.\mathbf{G} * \mathbf{F}\right|_{\mathbf{r}}+\left.\mathbf{G} * \mathbf{\Sigma} * \mathbf{u}\right|_{\mathbf{r}}
$$

Iterating indefinitely, one obtains :

$$
\begin{aligned}
\mathbf{u}(\mathbf{r})= & \left.\mathbf{G} * \mathbf{F}\right|_{\mathbf{r}}+\left.\mathbf{G} * \mathbf{\Sigma} * \mathbf{G} * \mathbf{F}\right|_{\mathbf{r}} \\
& +\left.\mathbf{G} * \mathbf{\Sigma} * \mathbf{G} * \mathbf{\Sigma} * \mathbf{G} * \mathbf{F}\right|_{\mathbf{r}}+\ldots
\end{aligned}
$$

Representing $\boldsymbol{\Sigma}$ as a series in the number $i$ of distinct scattering events :

$$
\boldsymbol{\Sigma}\left(\mathbf{r}, \mathbf{r}^{\prime}\right)=\sum_{i=1}^{\infty} \boldsymbol{\Sigma}^{(i)}\left(\mathbf{r}, \mathbf{r}^{\prime}\right)
$$

substituting in Eq. (23) and comparing to the formal solution from Eq. (11), it follows that :

$$
\begin{aligned}
& \boldsymbol{\Sigma}^{(1)}\left(\mathbf{r}, \mathbf{r}^{\prime}\right)=\left\langle\mathbf{T}^{\mathrm{OBJ}}\left(\mathbf{r}, \mathbf{r}^{\prime}\right)\right\rangle \\
& \boldsymbol{\Sigma}^{(2)}\left(\mathbf{r}, \mathbf{r}^{\prime}\right)=-\left.\left\langle\mathbf{T}^{O B J}\right\rangle * \mathbf{G}\left(\mathbf{r}_{1}, \mathbf{r}_{2}\right) *\left\langle\mathbf{T}^{O B J}\right\rangle\right|_{\left(\mathbf{r}, \mathbf{r}^{\prime}\right)}(25) \\
& \boldsymbol{\Sigma}^{(3)}\left(\mathbf{r}, \mathbf{r}^{\prime}\right)=\left.\left\langle\mathbf{T}^{O B J}\right\rangle * \mathbf{G} *\left\langle\mathbf{T}^{O B J}\right\rangle * \mathbf{G} *\left\langle\mathbf{T}^{O B J}\right\rangle\right|_{\left(\mathbf{r}, \mathbf{r}^{\prime}\right)}
\end{aligned}
$$

where integration over $\mathbf{r}_{1}$ and $\mathbf{r}_{2}$ in the multiple convolution is understood.

The expressions displayed above are particular for the case of only one particle in the solution, but they illustrate the basics of the formalism used in this paper. Now it is straightforward to generalize the procedure developed before to deal with a system where more than one object, or different types of particles are immersed in a fluid. 
Finally, at any volume fraction $\Phi$ of the solute particles, we compute self-consistently [7] the contribution $\boldsymbol{\Sigma}\left(\mathbf{r}, \mathbf{r}^{\prime}\right)$ of the suspended particles to the divergence of the full stress tensor of the fluid. This is accomplished by assuming linear superposition of the contributions of each particle to the self-energy of the fluid (assumption which is exact when there are no thermodynamic correlations among particles, as in the present work). Then, for a suspension of $N$ particles, one can replace $N-1$ of them with an effective medium described by the modified Oseen tensor $\mathcal{G}\left(\mathbf{r}, \mathbf{r}^{\prime}\right)$, which is a function of some $\boldsymbol{\Sigma}_{N-1}$ (see Eqs. (12,17,18)). Then, it follows that $\delta \boldsymbol{\Sigma}$, the self-energy increase due to the remaining particle is :

$$
\delta \boldsymbol{\Sigma}\left(\mathcal{G}\left(\boldsymbol{\Sigma}_{N-1}\right)\right)=\frac{\boldsymbol{\Sigma}_{N-1}}{N-1}
$$

The left-hand side of the equation can be evaluated as outlined for the case of one object in a fluid, but using the $\mathcal{G}$ propagator instead of $\mathbf{G}$ of the pure fluid. Thus we have obtained a self-consistent equation for calculating the full $\boldsymbol{\Sigma}_{N-1}$.

\section{B. Calculation of $\boldsymbol{\Sigma}\left(\mathbf{r}, \mathbf{r}^{\prime}\right)$ for a suspension of $\mathbf{N}_{S}$ spheres and $\mathrm{N}_{P}$ polymer chains}

We consider $\mathrm{N}_{S}$ fixed, rigid spheres, each of radius $a$, and $\mathrm{N}_{P}$ mobile, Gaussian polymer chains, each of length $L=n l$ ( $l=$ Kuhn length; $n=$ number of beads $)$, immersed in an incompressible fluid of viscosity $\eta_{0}$, in the regime of stationary flow.

The static velocity field in the suspension is described by the N-S equation together with stick boundary conditions :

$$
\begin{gathered}
-\eta_{0} \triangle \mathbf{v}(\mathbf{r})+\nabla p(\mathbf{r}) \quad=\mathbf{F}(\mathbf{r})+\sum_{\alpha, i \geq 1}^{\mathrm{N}_{P}, n} \delta\left(\mathbf{r}-\mathbf{R}_{\alpha i}\right) \boldsymbol{\sigma}_{\alpha i} \\
+\sum_{b=1}^{\mathrm{N}_{S}} \int d \Omega_{b} \delta\left(\mathbf{r}-\mathbf{R}_{b}\right) \boldsymbol{\sigma}_{b}\left(\Omega_{b}\right) \\
\nabla \cdot \mathbf{v}(\mathbf{r})=0 \\
\dot{\mathbf{R}}_{\alpha i}=\mathbf{u}_{\alpha}+\boldsymbol{\omega}_{\alpha} \times \mathbf{S}_{\alpha i}=\mathbf{v}\left(\mathbf{R}_{\alpha i}\right) \\
\dot{\mathbf{R}}_{b}=0=\mathbf{v}\left(\mathbf{R}_{b}\right)
\end{gathered}
$$

Here, the greek symbols $\alpha, \beta, \ldots$ are labeling the polymer chains and are running from 1 to $\mathrm{N}_{P}, i, j, k, \ldots$ are indices $(\in\{1, n\})$ for the beads on any arbitrary chain and $b, c \ldots$ are labels for the spheres (range $\overline{1, N_{S}}$ ) $. \mathbf{R}_{\alpha i}=\mathbf{R}_{\alpha}^{0}+\mathbf{S}_{\alpha i}$ is the position vector of the $i$-th bead of the chain $\alpha$, with $\mathbf{R}_{\alpha}^{0}$ denoting the position vector of the center of mass of the $\alpha$ chain and with $\mathbf{S}_{\alpha i}$ the position vector of the $i$-th bead with respect to the center of mass of $\alpha$.
$\mathbf{R}_{b}=\mathbf{R}_{b}^{0}+\mathbf{r}_{b}\left(\Omega_{b}\right)$ is the decomposition of the position vector of a point on the surface of the sphere $b$ in the position vector of its center of mass and the relative coordinate of the surface point in the center of mass frame $\left(\left|\mathbf{r}_{b}\right|=a\right.$, for any sphere); $\Omega$ indicates the orientation of the vector $\mathbf{r}_{b} . \boldsymbol{\sigma}_{\alpha i}$ and $\boldsymbol{\sigma}_{b}\left(\Omega_{b}\right)$ are the densities of force exerted by the $i$-th bead of chain $\alpha$ and by the sphere $b$ at the surface point $\mathbf{r}_{b}$, respectively, upon the fluid. When there is no risk of confusion, we will write only $\boldsymbol{\sigma}_{b}$, the dependence of $\Omega_{b}$ being understood. Finally, $\mathbf{F}(\mathbf{r})$ is some force acting at $\mathbf{r}$ that generates the flow (e.g.the effect of any sphere or polymer chain moving with uniform velocity).

From the boundary conditions for the polymer chains and because we are neglecting the inertial terms in the equations of motion for the polymers, the total force and torque acting upon any chain must vanish. We can write then :

$$
\begin{aligned}
-\sum_{i=1}^{n} \boldsymbol{\sigma}_{\alpha i} & =0 \\
-\sum_{i=1}^{n} \mathbf{S}_{\alpha i} \times \boldsymbol{\sigma}_{\alpha i} & =0 \quad, \alpha=\overline{1, \mathrm{~N}_{P}}
\end{aligned}
$$

The formal solution of Eq. (27) is :

$$
\begin{aligned}
\mathbf{v}(\mathbf{r})= & \left.\mathbf{G} * \mathbf{F}\right|_{\mathbf{r}}+\sum_{\alpha, i=1}^{\mathrm{N}_{P}, n} \mathbf{G}\left(\mathbf{r}-\mathbf{R}_{\alpha i}\right) \cdot \boldsymbol{\sigma}_{\alpha i} \\
& +\sum_{b=1}^{\mathrm{N}_{S}} \int d \Omega_{b} \mathbf{G}\left(\mathbf{r}-\mathbf{R}_{b}\right) \cdot \boldsymbol{\sigma}_{b}\left(\Omega_{b}\right)
\end{aligned}
$$

Using the boundary conditions (28) and the constraints (29) we can eliminate the unknown forces $\boldsymbol{\sigma}_{\alpha i}$ and $\boldsymbol{\sigma}_{b}$, to express the solution for the velocity field as a multiple scattering series in terms of the single-object flow propagator $\mathbf{T}_{\alpha}$ for the chain $\alpha$ and $\mathbf{T}_{b}$ for the $b$ sphere (see Appendix A for details) :

$$
\begin{aligned}
\mathbf{v}(\mathbf{r}) & =\left.\mathbf{G}(\mathbf{r}) * \mathbf{F}\right|_{\mathbf{r}}+\left.\sum_{\alpha, b} \mathbf{G} *\left\{\mathbf{T}_{\alpha}+\mathbf{T}_{b}\right\} * \mathbf{G} * \mathbf{F}\right|_{\mathbf{r}}+ \\
& +\left.\sum_{\alpha, b} \mathbf{G} *\left\{\mathbf{T}_{\alpha} * \mathbf{G} * \mathbf{T}_{b}+\mathbf{T}_{b} * \mathbf{G} * \mathbf{T}_{\alpha}\right\} * \mathbf{G} * \mathbf{F}\right|_{\mathbf{r}} \\
& +\left.\sum_{\alpha \neq \beta} \mathbf{G} * \mathbf{T}_{\alpha} * \mathbf{G} * \mathbf{T}_{\beta} * \mathbf{G} * \mathbf{F}\right|_{\mathbf{r}} \\
& +\left.\sum_{b \neq c} \mathbf{G} * \mathbf{T}_{b} * \mathbf{G} * \mathbf{T}_{c} * \mathbf{G} * \mathbf{F}\right|_{\mathbf{r}}+\ldots
\end{aligned}
$$

where the sum should be continued over all possible scattering sequences. In this expression, the first factor on the right-hand side represents the direct wave, the second contains the single scattering processes from only one polymer chain or one sphere, the third includes the sequences sphere-polymer and polymer-sphere,etc... . Any 
sequence of $\mathbf{T}$ operators is valid except those involving two consecutive scatterings off the same sphere or the same polymer (the exclusion constraint).

The macroscopic equation for the effective velocity field $\mathbf{u}(\mathbf{r})$ is retrieved by performing a configurational (position) average over the distribution of the particles, mathematically expressed as :

$$
\langle\cdot\rangle=\frac{1}{V^{\mathrm{N}_{P} \mathrm{~N}_{S}}} \int \prod_{\alpha} d \mathbf{R}_{\alpha}^{0} \int \prod_{b} d \mathbf{R}_{b}^{0}\langle\cdot\rangle_{\alpha i, \alpha j, \ldots}
$$

with the "0" superscript referring the center of mass and $<>_{\alpha i, \alpha j, \ldots}$ being an average over the distribution of the segments of the $\alpha$ chain about its center of mass. The probability distribution function is taken to be Gaussian (see [10]). Then $\mathbf{u}(\mathbf{r})$ is calculated from :

$$
\begin{aligned}
& \mathbf{u}(\mathbf{r})=\langle\mathbf{v}(\mathbf{r})\rangle=\mathbf{G} * \mathbf{F}+\sum_{\alpha, b} \mathbf{G} *\left\{\left\langle\mathbf{T}_{\alpha}\right\rangle+\left\langle\mathbf{T}_{b}\right\rangle\right\} * \mathbf{G} * \mathbf{F} \\
& +\sum_{\alpha, b} \mathbf{G} *\left\{\left\langle\mathbf{T}_{\alpha} * \mathbf{G} * \mathbf{T}_{b}\right\rangle+\left\langle\mathbf{T}_{b} * \mathbf{G} * \mathbf{T}_{\alpha}\right\rangle\right\} * \mathbf{G} * \mathbf{F} \\
& \quad+\sum_{\alpha \neq \beta} \mathbf{G} *\left\langle\mathbf{T}_{\alpha} * \mathbf{G} * \mathbf{T}_{\beta}\right\rangle * \mathbf{G} * \mathbf{F} \\
& +\sum_{b \neq c} \mathbf{G} *\left\langle\mathbf{T}_{b} * \mathbf{G} * \mathbf{T}_{c}\right\rangle * \mathbf{G} * \mathbf{F}+\ldots
\end{aligned}
$$

where all convolutions are actually functions of $\mathbf{r}$.

As outlined in the previous section, the meaningful physical quantity is the self-energy tensor $\boldsymbol{\Sigma}\left(\mathbf{r}, \mathbf{r}^{\prime}\right)$ defined as :

$$
\begin{aligned}
\boldsymbol{\Sigma} * \mathbf{u} & =\left\langle\sum_{\alpha, i} \delta\left(\mathbf{r}-\mathbf{R}_{\alpha i}\right) \boldsymbol{\sigma}_{\alpha i}+\sum_{b} \int d \Omega_{b} \delta\left(\mathbf{r}-\mathbf{R}_{b}\right) \boldsymbol{\sigma}_{b}\right\rangle \\
& =\boldsymbol{\Sigma}^{\mathbf{P O L}} * \mathbf{u}+\mathbf{\Sigma}^{\mathbf{S P}} * \mathbf{u}
\end{aligned}
$$

Next, the solution of the configurationally averaged Navier-Stokes equation is still Eq. (23), but now the total $\boldsymbol{\Sigma}$ includes the effects of both the polymers and the spheres. Expanding once again the self-energy in the number of distinct scattering events (i.e. the number of $\mathbf{T}_{\alpha, b}$ operators) and comparing the terms with the same number of $\mathbf{T}$ factors in Eqs. (23,33), it follows that :

$$
\begin{aligned}
& \boldsymbol{\Sigma}^{(1)}\left(\mathbf{r}, \mathbf{r}^{\prime}\right)=\sum_{\alpha}\left\langle\mathbf{T}_{\alpha}\right\rangle+\sum_{b}\left\langle\mathbf{T}_{b}\right\rangle \\
& \boldsymbol{\Sigma}^{(2)}\left(\mathbf{r}, \mathbf{r}^{\prime}\right)=\sum_{\alpha \neq \beta}\left\langle\mathbf{T}_{\alpha} * \mathbf{G} * \mathbf{T}_{\beta}\right\rangle+\sum_{b \neq c}\left\langle\mathbf{T}_{b} * \mathbf{G} * \mathbf{T}_{c}\right\rangle \\
& +\sum_{\alpha, b}\left\{\left\langle\mathbf{T}_{\alpha} * \mathbf{G} * \mathbf{T}_{b}\right\rangle+\left\langle\mathbf{T}_{b} * \mathbf{G} * \mathbf{T}_{\alpha}\right\rangle\right\} \\
& -\sum_{\alpha, \beta}\left\langle\mathbf{T}_{\alpha}\right\rangle * \mathbf{G} *\left\langle\mathbf{T}_{\beta}\right\rangle-\sum_{b, c}\left\langle\mathbf{T}_{b}\right\rangle * \mathbf{G} *\left\langle\mathbf{T}_{c}\right\rangle \\
& -\sum_{\alpha, b}\left\{\left\langle\mathbf{T}_{\alpha}\right\rangle * \mathbf{G} *\left\langle\mathbf{T}_{b}\right\rangle+\left\langle\mathbf{T}_{b}\right\rangle * \mathbf{G} *\left\langle\mathbf{T}_{\alpha}\right\rangle\right\}
\end{aligned}
$$

Assuming that the spheres do not rotate, we can derive the following expressions for the $\mathbf{T}_{\alpha, b}$ operators (see Appendix A) :

$$
\begin{aligned}
& \mathbf{T}_{\alpha}\left(\mathbf{r}, \mathbf{r}^{\prime}\right)=-\sum_{i, j}^{n} \delta\left(\mathbf{r}-\mathbf{R}_{\alpha i}\right)\left[\mathbf{K}^{-1}\left(\mathbf{S}_{\alpha i}, \mathbf{S}_{\alpha j}\right)\right. \\
& \left.-\sum_{l, l^{\prime}=1}^{n} \mathbf{K}^{-1}\left(\mathbf{S}_{\alpha i}, \mathbf{S}_{\alpha l}\right) \cdot \mathbf{g}_{t}^{-1} \cdot \mathbf{K}^{-1}\left(\mathbf{S}_{\alpha l^{\prime}}, \mathbf{S}_{\alpha j}\right)\right] \delta\left(\mathbf{r}-\mathbf{R}_{\alpha j}\right) \\
& + \text { rotational terms }+\ldots \\
& \mathbf{g}_{t}=\sum_{i, j}^{n} \mathbf{K}_{i j}^{-1} ; \quad \mathbf{g}_{t} \cdot \mathbf{g}_{t}^{-1}=\mathbb{1} \text {. } \\
& \mathbf{T}_{b}\left(\mathbf{r}-\mathbf{r}^{\prime}\right)=-\int d \Omega_{b} d \Omega_{b}^{\prime} \delta\left(\mathbf{r}-\mathbf{R}_{b}\right) \mathbf{K}_{b}^{-1}\left(\Omega_{b}, \Omega_{b}^{\prime}\right) \delta\left(\mathbf{r}^{\prime}-\mathbf{R}_{b}^{\prime}\right)
\end{aligned}
$$

in which $\mathbf{R}_{b}$ and $\mathbf{R}_{b}^{\prime}$ are the position vectors of separate points on the surface of the same sphere labeled $b$ and $\mathbf{K}_{\alpha}^{-1}$ and $\mathbf{K}_{b}^{-1}$ are the generalized inverse operators for the single polymer chain and the single sphere [12, 15, defined by :

$$
\begin{array}{r}
\sum_{j=1}^{n} \mathbf{K}^{-1}\left(\mathbf{S}_{i}, \mathbf{S}_{j}\right) \cdot \mathbf{G}\left(\mathbf{S}_{j}-\mathbf{S}_{k}\right)=\mathbb{1} \delta_{i k} \\
\int d \Omega_{b}^{\prime \prime} \mathbf{K}^{-1}\left(\Omega_{b}, \Omega_{b}^{\prime \prime}\right) \cdot \mathbf{G}\left(\mathbf{r}_{b}\left(\Omega_{b}^{\prime \prime}\right)-\mathbf{r}_{b}\left(\Omega_{b}^{\prime}\right)\right)=\mathbb{1} \delta\left(\Omega_{b}-\Omega_{b}^{\prime}\right)
\end{array}
$$

To avoid an increasingly intricate notation, we will adopt the following short-hand notation :

$$
\begin{aligned}
\mathbf{K}^{-1}\left(\mathbf{S}_{\alpha i}, \mathbf{S}_{\alpha j}\right) & \rightarrow \mathbf{K}_{\alpha i, \alpha j}^{-1} \\
\mathbf{K}^{-1}\left(\Omega_{b}, \Omega_{b}^{\prime}\right) & \rightarrow \mathbf{K}_{b, b^{\prime}}^{-1} \\
\mathbf{G}\left(\mathbf{R}_{\alpha j}-\mathbf{R}_{b}\right) & \rightarrow \mathbf{G}_{\alpha j, b}
\end{aligned}
$$

The Einstein summation convention over repeated indices is implied everywhere but where explicitly not followed. We also drop the index $\alpha$ when referring to quantities not specifically depending on a particular polymer chain.

Some remarks are necessary before embarking upon some concrete calculations. In the limit of our static description of the suspension of polymer chains and spheres, Eqs. (35) are exact, for noninteracting as well as for interacting objects, and will describe the dynamics of the suspension with any degree of accuracy, although practically it may require a strenuous effort. To obtain analytical results, we are limiting ourselves to the case of noninteracting polymers and spheres and, further, we will approximate the total self-energy tensor $\boldsymbol{\Sigma}\left(\mathbf{r}, \mathbf{r}^{\prime}\right)$ with the 
first term in the expansion (35) that contains the contribution from single scattering events :

$$
\boldsymbol{\Sigma}\left(\mathbf{r}, \mathbf{r}^{\prime}\right) \simeq \boldsymbol{\Sigma}^{(1)}\left(\mathbf{r}, \mathbf{r}^{\prime}\right)
$$

but, for the convenience of notation we will still denote it by $\boldsymbol{\Sigma}\left(\mathbf{r}, \mathbf{r}^{\prime}\right)$. Actually, for the noninteracting situation, this is the leading term as can be seen from Eq. (35) by breaking the averages, with the contribution from higher order processes vanishing due to the lack of correlations among particles.

As already pointed out, $\boldsymbol{\Sigma}(\mathbf{k})$ contains the sought information about the transport properties of the suspension. To evaluate the self-energy, first we take the configurational average (32) over the $\mathbf{T}$ operators in Eq. (37), then we Fourier transform their expressions, obtaining :

$$
\begin{aligned}
\left\langle\mathbf{T}_{\alpha}(\mathbf{k})\right\rangle & =-\frac{1}{V} \sum_{i, j \geq 1}^{n}\left\langle\operatorname { e x p } ( i \mathbf { k } \cdot ( \mathbf { S } _ { i } - \mathbf { S } _ { j } ) ) \left[\mathbf{K}_{\alpha i, \alpha j}^{-1}\right.\right. \\
& \left.\left.-\sum_{l, l^{\prime} \geq 1}^{n} \mathbf{K}_{\alpha i, \alpha l}^{-1} \cdot \mathbf{g}_{t}^{-1} \cdot \mathbf{K}_{\alpha l^{\prime}, \alpha j}^{-1}\right]\right\rangle_{i, j} \\
\left\langle\mathbf{T}_{b}(\mathbf{k})\right\rangle & =-\frac{1}{V} \int d \Omega_{b} d \Omega_{b}^{\prime} \mathbf{K}_{b, b^{\prime}}^{-1} \exp \left[i \mathbf{k} \cdot\left(\mathbf{r}_{b}-\mathbf{r}_{b}^{\prime}\right)\right]
\end{aligned}
$$

The preaverage over $\mathbf{K}^{-1}$ part produces terms like $\left\langle\mathbf{K}_{i j}^{-1}\right\rangle_{i j}\left\langle\exp \left(i \mathbf{k} \cdot\left(\mathbf{S}_{i}-\mathbf{S}_{j}\right)\right)\right\rangle_{i j}$-with the $i, j$ indices signifying an average over the distribution of the segments of any chain - so $\boldsymbol{\Sigma}(\mathbf{k})$ can be written as :

$$
\begin{aligned}
& \boldsymbol{\Sigma}(\mathbf{k}) \simeq \boldsymbol{\Sigma}^{(1)}(\mathbf{k})=-c^{\mathrm{POL}} \sum_{i, j \geq 1}^{n}\left\langle\exp \left(i \mathbf{k} \cdot\left(\mathbf{S}_{i}-\mathbf{S}_{j}\right)\right)\right\rangle_{i j} \\
& \times\left[\left\langle\mathbf{K}_{i j}^{-1}\right\rangle_{i j}-\left\langle\mathbf{g}_{t}^{-1}\right\rangle_{i j} \cdot \sum_{l, l^{\prime} \geq 1}^{n}\left\langle\mathbf{K}_{i l}^{-1}\right\rangle_{i l} \cdot\left\langle\mathbf{K}_{l^{\prime} j}^{-1}\right\rangle_{l^{\prime} j}\right] \\
& -c^{\mathrm{SP}} \int d \Omega d \Omega^{\prime} \mathbf{K}^{-1}\left(\Omega, \Omega^{\prime}\right) \exp \left[i \mathbf{k} \cdot\left(\mathbf{r}(\Omega)-\mathbf{r}^{\prime}\left(\Omega^{\prime}\right)\right)\right]
\end{aligned}
$$

where $\mathbf{r}(\Omega), \mathbf{r}^{\prime}\left(\Omega^{\prime}\right)$ are two generic points on the surface of any sphere and $c^{\mathrm{POL}}=\frac{\mathrm{N}_{P}}{V}, c^{\mathrm{SP}}=\frac{\mathrm{N}_{S}}{V}$ are the concentrations of the polymers and of the spheres, respectively.

\section{CALCULATION OF $\boldsymbol{\Sigma}(\mathrm{k})$ AND EFFECTIVE MEDIUM THEORY FOR SOLUTIONS OF SPHERES OR POLYMERS ONLY}

In this part of the paper, the problems of constructing the effective media in the separate cases of a suspension of spheres and a polymer dispersion are addressed.

\section{A. Calculation of $\mathbf{W}(\mathbf{k})$ for $\mathbf{N}_{S}$ spheres immersed in a fluid : a review}

This problem is well documented in the literature [7, 15 so we will quote the main results we need. The system consists in $\mathrm{N}_{S}$, rigid, penetrable spheres of radius $a$, immersed in an incompressible,newtonian fluid of viscosity $\eta_{0}$. All spheres but one, which is moving at constant velocity and generates the flow, are fixed. The suspension is assumed stationary.

A remark should be made regarding the notation. Because we want to distinguish this particular system from the others ,we will denote the self-energy of the fluid in the presence of the spheres only by $\mathbf{W}^{S P}$ instead of $\boldsymbol{\Sigma}^{S P}$.

Along the lines previously exposed,we can map the suspension averaged over the uniform distribution of the spheres to an effective fluid of viscosity $\eta_{S P}^{\text {eff }}$ where the disturbances are propagated by the modified Oseen tensor $\mathcal{G}^{S P}(\mathbf{k})$ :

$$
\mathcal{G}^{S P}(\mathbf{k})=\frac{\mathbb{1}-\hat{\mathbf{k}} \hat{\mathbf{k}}}{\eta_{0} k^{2}-W_{\perp}^{S P}(\mathbf{k})}
$$

with the transverse part of the self-energy computed from Eq. (16).

In order to solve self-consistently for $\mathbf{W}^{S P}(\mathbf{k})$ we expand its transverse part, in the small $\mathbf{k}$ limit :

$$
W_{\perp}^{S P}(\mathbf{k})=\left(-\eta_{0} \xi_{S P}^{-2}-\eta_{0} W_{1}^{S P}\left(\Phi^{S P}\right) k^{2}\right)
$$

Then the modified Oseen propagator becomes :

$$
\begin{aligned}
& \mathcal{G}^{S P}(\mathbf{k})= \frac{\mathbb{1}-\hat{\mathbf{k}} \hat{\mathbf{k}}}{\eta_{0}\left(1+W_{1}^{S P}\right)\left(k^{2}+\xi^{\prime-2}\right)} \\
& \xi_{S P}^{\prime-2}=\frac{\xi_{S P}^{-2}}{1+W_{1}^{S P}}
\end{aligned}
$$

where $W_{1}^{S P}$ is some function of $\Phi^{S P}$. All other symbols were already defined.

$\mathbf{W}^{S P}(\mathbf{k})$ has the following form (see Appendix B) :

$$
\begin{aligned}
& \mathbf{W}^{S P}(\mathbf{k})=-6 \pi \eta_{0} a c^{S P} \frac{1}{\mathrm{I}_{1 / 2}\left(\frac{a}{\xi^{\prime}}\right) \mathrm{K}_{1 / 2}\left(\frac{a}{\xi^{\prime}}\right)}\left(1+W_{1}^{S P}\right) \mathbb{1}+ \\
& +\frac{3}{2} \eta_{0} \Phi^{S P}\left(1+W_{1}^{S P}\right) k^{2}\left[\frac{1}{\mathrm{I}_{1 / 2}\left(\frac{a}{\xi^{\prime}}\right) \mathrm{K}_{1 / 2}\left(\frac{a}{\xi^{\prime}}\right)}\right. \\
& \left.-\frac{8}{9 \mathrm{I}_{3 / 2}\left(\frac{a}{\xi^{\prime}}\right) \mathrm{K}_{3 / 2}\left(\frac{a}{\xi^{\prime}}\right)}-\frac{2}{5 \mathrm{I}_{1 / 2}\left(\frac{a}{\xi^{\prime}}\right) \mathrm{K}_{5 / 2}\left(\frac{a}{\xi^{\prime}}\right)}\right] \mathbb{1} \\
& +\frac{3}{2} \eta_{0} \Phi^{S P}\left(1+W_{1}^{S P}\right) k^{2}\left[\frac{4}{27 \mathrm{I}_{3 / 2}\left(\frac{a}{\xi^{\prime}}\right) \mathrm{K}_{3 / 2}\left(\frac{a}{\xi^{\prime}}\right)}\right. \\
& \left.+\frac{2}{5 \mathrm{I}_{1 / 2}\left(\frac{a}{\xi^{\prime}}\right) \mathrm{K}_{5 / 2}\left(\frac{a}{\xi^{\prime}}\right)}\right] \hat{\mathbf{z}} \hat{\mathbf{z}}
\end{aligned}
$$


where I and $\mathrm{K}$ are the modified Bessel functions of the first kind, $\Phi^{S P}=\frac{4 \pi a^{3}}{3} c^{S P}$ is the volume fraction of spheres and $\hat{\mathbf{z}}$ is the versor of the $\mathrm{Oz}$ direction. Projecting out the transverse part of $\mathbf{W}^{S P}(\mathbf{k})$ by dotting in with $(\mathbb{1}-\hat{\mathbf{z}} \hat{\mathbf{z}})$ and comparing the result with the expansion (45), one arrives at the following set of equations for $x=\frac{a}{\xi^{\prime}}$ and $W_{1}^{S P}$ :

$$
\begin{aligned}
x^{2} & =\frac{9}{2} \Phi^{S P} \frac{1}{\mathrm{I}_{1 / 2}(x) \mathrm{K}_{1 / 2}(x)} \\
W_{1}^{S P} & \left(\Phi^{S P}\right)=\left\{1+\frac{3}{2} \Phi^{S P}\left[\frac{1}{\mathrm{I}_{1 / 2}(x) \mathrm{K}_{1 / 2}(x)}\right.\right. \\
& \left.\left.-\frac{8}{9 \mathrm{I}_{3 / 2}(x) \mathrm{K}_{3 / 2}(x)}-\frac{2}{5 \mathrm{I}_{1 / 2}(x) \mathrm{K}_{5 / 2}(x)}\right]\right\}^{-1}-1
\end{aligned}
$$

These equations can be solved numerically and $x\left(\Phi^{S P}\right)$ and $W_{1}^{S P}\left(\Phi^{S P}\right)$ calculated for any values of $0 \leq \Phi^{S P}<$ 1 , thus leading to the required $\mathbf{W}^{S P}(\mathbf{k})$. As $\Phi^{S P} \rightarrow 0$, $W_{1}^{S P}$ decreases to zero and also, from the structure of Eq. (48), one expects a divergent behavior of $W_{1}^{S P}$ as $\Phi^{S P}$ increases beyond a certain limit. The effective viscosity $\eta_{\mathrm{SP}}$ of the medium containing the spheres and the translational friction coefficient $\boldsymbol{\zeta}_{t}$ of the moving sphere are obtained from :

$$
\begin{aligned}
& \frac{\eta_{\mathrm{SP}}-\eta_{0}}{\eta_{0}}=W_{1}^{S P}\left(\Phi^{S P}\right) \\
& \boldsymbol{\zeta}_{t}=\zeta_{t} \mathbb{1}=-\frac{1}{c^{S P}} \mathbf{W}^{S P}(k=0)=6 \pi a \eta_{0} \\
& \times \frac{1}{\mathrm{I}_{1 / 2}\left(x\left(\Phi^{S P}\right)\right) \mathrm{K}_{1 / 2}\left(x\left(\Phi^{S P}\right)\right)}\left(1+W_{1}^{S P}\left(\Phi^{S P}\right)\right) \mathbb{1}
\end{aligned}
$$

One can derive the above expression for the friction coefficient by applying the general analysis from Appendix A to our particular case of a suspension of $N-1$ fixed and one uniformly moving spheres . Then, the relation between the total average force exerted upon the mobile sphere by the fluid and its velocity will be given by Eq. (A6) without the polymer term :

$$
\begin{aligned}
\left\langle\int d \Omega \boldsymbol{\sigma}(\Omega)\right\rangle= & \left\langle\int d \Omega d \Omega^{\prime} \boldsymbol{T}\left(\Omega, \Omega^{\prime}\right)\right\rangle \cdot \mathbf{u}= \\
& \left\langle\int d \Omega d \Omega^{\prime} \mathcal{K}^{-1}\left(\Omega, \Omega^{\prime}\right)\right\rangle \cdot \mathbf{u}=\boldsymbol{\zeta}_{t} \cdot \mathbf{u}
\end{aligned}
$$

Note that now $\mathcal{K}^{-1}$ is the generalized inverse of $\mathcal{G}$, to account for the presence of the fixed spheres in the solution. Comparing then to the sphere contribution to the self-energy from Eq. (43) and making use of (B7,,B18) one can recover the formula for $\boldsymbol{\zeta}_{t}$ displayed in (49). When the filling fraction of the background spheres approaches zero, $W_{1}^{S P} \rightarrow 0$ and one recovers the Stokes result for the friction coefficient $\zeta_{\mathbf{t}}$ of one sphere.
As stated before, the readily measurable quantity we calculated is the relative diffusion coefficient of the mobile sphere (relative to the bare diffusion constant), introduced in (21). From (49) we get immediately :

$$
\begin{aligned}
\mathbf{D}^{S P}=D^{S P} \mathbb{1} & =\mathrm{I}_{1 / 2}\left(x\left(\Phi^{S P}\right)\right) \mathrm{K}_{1 / 2}\left(x\left(\Phi^{S P}\right)\right) \\
& \times \frac{1}{1+W_{1}^{S P}\left(\Phi^{S P}\right)} \mathbb{1}
\end{aligned}
$$

in which $x\left(\Phi^{S P}\right)$ and $W_{1}^{S P}\left(\Phi^{S P}\right)$ are calculated from (48) for a given fraction $\Phi^{S P}$.

\section{B. Calculation of the self-energy for a solution of $\mathbf{N}_{P}$ noninteracting polymer chains}

We consider $\mathrm{N}_{P}$ Gaussian, noninteracting, free moving polymer chains, each of length $L=n l$, dispersed in an incompressible, newtonian fluid of viscosity $\eta_{0}$. Some external force $\mathbf{F}(\mathbf{r})$ generates the velocity flow.

Once again, we will use the notation $\mathbf{W}$ for the selfenergy of the fluid when only the polymers are present.

Following the derivation in Sec. IIB, we can write the Navier-Stokes equation for the velocity field $\mathbf{v}(\mathbf{r})$ similar to (27) - but no spheres present - with the stick boundary conditions (28). Averaging the N-S equation over the configuration of the polymer chains and introducing the self-energy of the fluid $\mathbf{W}$ as in (34), we can write the solution for the averaged velocity $\mathbf{u}(\mathbf{r})=\mathcal{G}^{P O L} * \mathbf{F}$ (see Eqs. (12, 17, 18)), where the force propagator $\mathcal{G}^{P O L}$ is given by :

$$
\begin{aligned}
\mathcal{G}^{P O L}= & \frac{\mathbb{1}-\hat{\mathbf{k}} \hat{\mathbf{k}}}{\eta_{0} k^{2}-W_{\perp}^{P O L}(\mathbf{k})}= \\
& \frac{\mathbb{1}-\hat{\mathbf{k}} \hat{\mathbf{k}}}{\eta_{0}\left(1+W_{1}^{P O L}\right)\left(k^{2}+\xi_{P O L}^{\prime}{ }^{-2}\right)} \\
\xi_{P O L}^{\prime}{ }^{-2}= & \frac{\xi_{P O L}^{-2}}{1+W_{1}^{P O L}}
\end{aligned}
$$

where, in the limit of $k \rightarrow 0$, the following approximation was made for the transverse part of the self-energy :

$$
W_{\perp}^{P O L}(\mathbf{k})=-\eta_{0} \xi_{P O L}^{-2}-\eta_{0} W_{1}^{P O L}\left(\Phi^{P O L}\right) k^{2}
$$

Our goal is to find $\mathbf{W}^{P O L}(\mathbf{k})$ self-consistently, for any volume fraction $\Phi^{P O L}$. If $\mathbf{W}^{P O L}(\mathbf{k})$ were the self-energy of the fluid containing $\mathrm{N}_{P}-1$ chains, the contribution of one more chain added would be (similar to (43), but with $c^{P O L}=1 / V$ and no spheres) :

$$
\frac{\mathbf{W}^{P O L}(\mathbf{k})}{\mathrm{N}_{P}-1}=-\frac{1}{V} \sum_{i, j \geq 1}^{n}\left\langle\exp \left[i \mathbf{k} \cdot\left(\mathbf{S}_{i}-\mathbf{S}_{j}\right)\right]\right\rangle_{i j}
$$




$$
\begin{aligned}
& {\left[\left\langle\mathcal{K}_{i j}^{-1}\right\rangle_{i j}-\left\langle\mathbf{g}_{t}^{-1}\right\rangle_{i j} \cdot \sum_{l, l^{\prime} \geq 1}^{n}\left\langle\mathcal{K}_{i l}^{-1}\right\rangle_{i l} \cdot\left\langle\mathcal{K}_{l^{\prime} j}^{-1}\right\rangle_{l^{\prime} j}\right]} \\
& \mathbf{g}_{t}=\sum_{i j}^{n} \mathcal{K}_{i j}^{-1} ; \mathbf{g}_{t}^{-1} \cdot \mathbf{g}_{t}=\mathbb{1}
\end{aligned}
$$

Here, in contrast to (43), $\mathcal{K}^{-1}$ signifies the generalized inverse of the modified Oseen tensor $\mathcal{G}$ because we imagined the last chain immersed in the effective medium created by the initial pure fluid plus $\mathrm{N}_{P}-1$ polymer chains and the force propagator should be changed correspondingly.

The calculations are rather laborious and are detailed in Appendix C. In the hydrodynamic limit of long wavelengths, the expression of $\mathbf{W}^{P O L}(\mathbf{k})$ is :

$$
\begin{aligned}
\mathbf{W}^{P O L}(\mathbf{k}) & =-\frac{9}{\pi} \eta_{0} k^{2} \Phi^{P O L}\left(1+W_{1}^{P O L}\right) \mathrm{Q}(\beta) \mathbb{1} \\
\mathrm{Q}(\beta) & =\frac{1}{\beta} \log \left(1+\frac{\beta}{\sqrt{\pi}}\right)+\frac{1}{\sqrt{\pi}}+\frac{\beta}{2 \pi}
\end{aligned}
$$

Symbols have the following meanings: $\Phi^{P O L}=\frac{4 \pi R_{g}^{3}}{3} c^{P O L}$ is the volume fraction of the polymers ; $R_{g}=\sqrt{\frac{L l}{6}}$ is the radius of gyration ; $\beta\left(\Phi^{P O L}\right)=\frac{R_{g}}{\xi^{\prime}\left(\Phi^{P O L}\right)}$ with $\xi^{\prime}$ the screening length in the effective medium, given by (52). In obtaining the previous relation we used the KirkwoodRiseman approximation that amounts to consider only the diagonal terms in the Fourier expansion of $\mathcal{G}^{-1}\left(s, s^{\prime}\right)$ ( $s$ is the arclength along the polymer chain), as detailed in Appendix C. We believe this approximation is physically justifiable because we are working in the limit of long chains, when the off-diagonal terms are small.

To get the self-consistent equations for the unknowns $\beta\left(\Phi^{P O L}\right)$ and $W_{1}^{P O L}\left(\Phi^{P O L}\right)$, we take the transverse part of $\mathbf{W}^{P O L}(\mathbf{k})$ by dotting in $(\mathbb{1}-\hat{\mathbf{k}} \hat{\mathbf{k}})$ and then we equate it with the expansion (53) to arrive at :

$$
\begin{aligned}
\beta\left(\Phi^{P O L}\right) & =0 \\
W_{1}^{P O L}\left(\Phi^{P O L}\right) & =\frac{1}{1-\frac{9}{\pi} \Phi^{P O L} \mathrm{Q}(0)}-1
\end{aligned}
$$

which gives $W_{1}^{P O L}$ as an analytic function of the volume fraction $\Phi^{P O L}$. Also it is interesting to remark that $\beta\left(=\frac{R_{g}}{a}\right)=0$ implies that the hydrodynamic screening is absent in the limit of $k \rightarrow 0$ (observation that is actually manifest from the structure of $(55))$. The function $\mathrm{Q}(\beta)$ is defined in Eq. (55).

We can calculate the viscosity of the effective medium $\eta_{\mathrm{POL}}$ (using (19)) and the diffusion coefficient $D^{P O L}$ of a polymer chain as follows :

$$
\frac{\eta_{\mathrm{POL}}\left(\Phi^{P O L}\right)-\eta_{0}}{\eta_{0}}=W_{1}^{P O L}
$$

$$
\begin{aligned}
D^{P O L}\left(\Phi^{P O L}\right) & =\frac{\zeta_{t}^{K R}}{\zeta_{t}^{P O L}\left(\Phi^{P O L}\right)} \\
& =\frac{1}{1+W_{1}^{P O L}}=1-\frac{18}{\pi \sqrt{\pi}} \Phi^{P O L}
\end{aligned}
$$

where we employed $\mathrm{Q}(0)=\frac{2}{\sqrt{\pi}}$ and $\zeta_{t}^{K R}=(9 \pi \sqrt{\pi} / 4) \eta_{0} R_{g}$ is the Kirkwood-Riseman friction coefficient for the nonfree-draining limit.

Limiting values for $D^{P O L}$ and $\eta_{\mathrm{POL}}$ can be obtained for small and large polymer filling fractions $\Phi^{P O L}$ :

$$
\begin{aligned}
\Phi^{P O L} \ll & 1: \\
& \frac{\eta_{\mathrm{POL}}-\eta_{0}}{\eta_{0}} \rightarrow \frac{24}{\sqrt{\pi}} R_{g}^{3} \frac{1}{V} \\
\Phi^{P O L}(\text { large })< & 1: \\
& \frac{D_{\mathrm{POL}}-\eta_{0}}{\eta_{0}} \rightarrow \infty \text { as } \Phi^{P O L} \rightarrow 0.309 \ldots \\
& D^{P O L} \rightarrow 0
\end{aligned}
$$

We remark that for low $\Phi^{P O L}$ the result for the viscosity is twice that of Kirkwood-Riseman [14 for one chain because we neglect the rotational terms in calculating $\mathbf{W}^{P O L}(\mathbf{k})$. It is also notable that the constraint of the positivity of $W_{1}^{P O L}$ in this effective medium approach leads to the divergence of the viscosity and of the friction coefficient, which occurs at $\Phi_{D I V}^{P O L} \simeq 0.309$. This divergence is attributed to the rigid-body dynamics assumed for the polymer.

\section{RESULTS FOR SPECIFIC EXAMPLES}

In this section we apply the above theory to two types of mixed polymers-spheres suspensions :

$\mathbf{S} / \mathbf{P}$ system : one mobile sphere among other fixed, rigid ones, in a polymer solution.

$\mathbf{P} / \mathbf{S}$ system : free moving polymer chains in a suspension of fixed spheres.

In addition, to gradually develop the theory we start by explicitly investigating two particular cases of the general models just mentioned, namely :

1S/P system : one mobile sphere inside a polymer solution.

1P/S system : one polymer chain moving in a suspension of fixed spheres. 


\section{A. One sphere moving in a suspension of polymers}

In this example we consider a sphere of radius $a$ moving uniformly with the velocity $\mathbf{v}_{0}$ inside a suspension of $\mathrm{N}_{P}$ non-interacting, Gaussian, free moving polymer chains of length $L$. The flow of the fluid is generated by the moving sphere. The motion of the particles is coupled to the motion of the solvent by stick boundary conditions. We also mention that the total force and torque acting upon each polymer chain are zero.

Similar to Eq. (27), the equation of motion for the fluid and the boundary conditions can be written as :

$$
\begin{gathered}
-\eta_{0} \Delta \mathbf{v}(\mathbf{r})+\nabla p(\mathbf{r})=\mathbf{F}_{0}(\mathbf{r})+\sum_{\alpha, i}^{N P, n} \delta\left(\mathbf{r}-\mathbf{R}_{\alpha i}\right) \boldsymbol{\sigma}_{\alpha i} \\
\nabla \cdot \mathbf{v}(\mathbf{r})=0 \\
\dot{\mathbf{R}}_{\alpha i}=\mathbf{v}\left(\mathbf{R}_{\alpha i}\right)
\end{gathered}
$$

in which $\mathbf{F}_{0}(\mathbf{r})$ is the force exerted by the mobile sphere upon the fluid given by :

$$
\mathbf{F}_{0}(\mathbf{r})=\int d \Omega_{0} \delta\left(\mathbf{r}-\mathbf{R}_{0}\right) \boldsymbol{\sigma}\left(\Omega_{0}\right)
$$

Averaging the Navier-Stokes equation over the distribution of the polymer chains and introducing the selfenergy of the solution :

$$
\boldsymbol{\Sigma}\left(\mathbf{r}, \mathbf{r}^{\prime}\right)=\mathbf{W}^{P O L}\left(\mathbf{r}, \mathbf{r}^{\prime}\right)=\left\langle\sum_{\alpha, i \geq 1}^{N P, n} \delta\left(\mathbf{r}-\mathbf{R}_{\alpha i}\right) \boldsymbol{\sigma}_{\alpha i}\right\rangle
$$

we find the formal solution (following Eqs. (12,18) :

$$
\begin{aligned}
\mathbf{v}(\mathbf{r}) & =\left.\mathcal{G} * \mathbf{F}_{0}\right|_{\mathbf{r}} \\
\mathcal{G}(\mathbf{k}) & =\frac{\mathbb{1}-\hat{\mathbf{k}} \hat{\mathbf{k}}}{\eta_{0} k^{2}-W_{\perp}^{P O L}(\mathbf{k})}
\end{aligned}
$$

and we succeeded in transforming the initial problem in the Stokes motion of a single sphere in a fluid of viscosity $\eta_{\text {POL }}$. Because only the polymers are contributing to the effective viscosity of the solution, $\eta^{\text {eff }}$ is given by (19,57) :

$$
\frac{\eta^{\mathrm{eff}}-\eta_{0}}{\eta_{0}}=W_{1}^{P O L}=\frac{1}{1-\frac{18}{\pi \sqrt{\pi}} \Phi^{P O L}}-1
$$

As it was shown in the previous section-Eq. (56) the hydrodynamic screening is absent for a suspension of polymers. In this problem, the sphere does not contribute to the self-energy of the fluid, which implies that the hydrodynamic interactions are still unscreened. Then the translational friction coefficient of the moving sphere is just the Stokes result :

$$
\boldsymbol{\zeta}_{t}=6 \pi a \eta^{\mathrm{eff}} \mathbb{1}
$$

with $\eta^{\text {eff }}=\eta_{0}\left(1+W_{1}^{P O L}\right)$. The translational diffusion coefficient as defined in (21) reads then :

$$
D\left(\Phi^{P O L}\right)=\frac{6 \pi a \eta_{0}}{\zeta_{t}}=1-\frac{18}{\pi \sqrt{\pi}} \Phi^{P O L}
$$

It is noteworthy that in this case there is no size coupling (no $t=\frac{R_{g}}{a}$ dependence) between the dynamics of the polymer chains and the dynamics of the moving sphere. The diffusion coefficient vanishes and the viscosity diverges at $\Phi^{P O L} \simeq 0.31$.

\section{B. One polymer chain immersed in a suspension of fixed spheres}

This time we consider a free polymer chain of length $L$, immersed in a suspension of $\mathrm{N}_{S}$ rigid, fixed, uniformly distributed spheres of radius $a$. There are no interactions other than hydrodynamic and the flow is generated by some external force field $\mathbf{F}(\mathbf{r})$.

The velocity field is described by another variant of N-S Eq. (27), with only one chain present :

$$
\begin{aligned}
-\eta_{0} \Delta \mathbf{v}(\mathbf{r})+\nabla p(\mathbf{r})= & \mathbf{F}(\mathbf{r})+\sum_{i=1}^{n} \delta\left(\mathbf{r}-\mathbf{R}_{i}\right) \boldsymbol{\sigma}_{i} \\
& +\sum_{b=1}^{N_{S}} \int d \Omega_{b} \delta\left(\mathbf{r}-\mathbf{R}_{b}\right) \boldsymbol{\sigma}_{b}
\end{aligned}
$$

where all the symbols have the known meanings.

Taking the configurational average of the equation above and introducing the self-energy tensors $\boldsymbol{\Sigma}^{P O L}\left(\mathbf{r}, \mathbf{r}^{\prime}\right)$ and $\mathbf{W}^{S P}\left(\mathbf{r}, \mathbf{r}^{\prime}\right)$ (see (34)) related to the influence of the polymer chain and of the spheres on the fluid, we can cast the Navier-Stokes equation for the averaged velocity $\mathbf{u}(\mathbf{r})=\langle\mathbf{v}(\mathbf{r})\rangle$ in the following form :

$$
-\eta_{0} \triangle \mathbf{u}(\mathbf{r})+\langle\nabla p(\mathbf{r})\rangle-\left.\mathbf{W}^{S P} * \mathbf{u}\right|_{\mathbf{r}}=\mathbf{F}(\mathbf{r})+\left.\boldsymbol{\Sigma}^{P O L} * \mathbf{u}\right|_{\mathbf{r}}
$$

the formal solution of which being (see Section II) :

$$
\mathbf{u}(\mathbf{r})=\left.\mathcal{G} * \mathbf{F}\right|_{\mathbf{r}}+\left.\mathcal{G} * \boldsymbol{\Sigma}^{P O L} * \mathcal{G} * \mathbf{F}\right|_{\mathbf{r}}+\ldots
$$

Here $*$ is the usual convolution operator and $\mathcal{G}\left(\mathbf{r}, \mathbf{r}^{\prime}\right)$ is the effective Oseen tensor modified to account for the influence of the spheres. Its expression is given by 46 ) using the expansion for small $k(45)$. In this way our 
starting problem was reduced to studying the stationary dynamics of a polymer chain in an effective solution where the force propagator is $\mathcal{G}(\mathbf{k})$. Following the results of Sec. IIB B Eqs. (40, 35, 42, 43) - and noticing that we must make the changes $c^{P O L}=\frac{1}{V}, \mathbf{G} \rightarrow \mathcal{G}$ and $\boldsymbol{K}^{-1} \rightarrow \mathcal{K}^{-1}$, we find the solution of $\boldsymbol{\Sigma}^{P O L}(\mathbf{k})$ in first order in the number of scattering events :

$$
\begin{aligned}
\boldsymbol{\Sigma}^{P O L}(\mathbf{k})= & -\frac{1}{V} \sum_{i, j \geq 1}^{n}\left\langle\exp \left[i \mathbf{k} \cdot\left(\mathbf{S}_{i}-\mathbf{S}_{j}\right)\right]\right\rangle_{i j}\left[\left\langle\mathcal{K}_{i j}^{-1}\right\rangle_{i j}\right. \\
& \left.-\left\langle\mathbf{g}_{t}^{-1}\right\rangle_{i j} \cdot \sum_{l, l^{\prime}}^{n}\left\langle\mathcal{K}_{i l}^{-1}\right\rangle_{i l} \cdot\left\langle\mathcal{K}_{l^{\prime} j}^{-1}\right\rangle_{l^{\prime} j}\right]
\end{aligned}
$$

Note that $\mathcal{K}^{-1}$ is the generalized inverse of $\mathcal{G}$ :

$$
\sum_{j=1}^{n} \mathcal{K}_{i j}^{-1} \cdot \mathcal{G}_{j k}=\mathbb{1} \delta_{i k}
$$

Remembering that the effective medium has replaced the spheres and the pure fluid, we can repeat the derivation from Appendix $\mathrm{C}$ to get an explicit form for $\boldsymbol{\Sigma}^{P O L}(\mathbf{k})$, with the changes $W_{1}^{P O L} \rightarrow W_{1}^{S P}$ and $c^{P O L}=\frac{1}{V}$ :

$$
\begin{gathered}
\boldsymbol{\Sigma}^{P O L}(\mathbf{k})=-\frac{9}{\pi} \eta_{0} \frac{4 \pi R_{g}^{3}}{3} \frac{1}{V}\left(1+W_{1}^{S P}\right) \mathrm{Q}\left(\beta_{S P}\right) k^{2} \mathbb{1} \\
\beta_{S P}=\frac{R_{g}}{\xi_{S P}^{\prime}}
\end{gathered}
$$

where $\xi_{S P}^{\prime}$ and $W_{1}^{S P}$ were defined in Sec. III A.

To evidentiate the important dimensionless variables of this model, we introduce the parameters $t=\frac{R_{g}}{a}$ and $x_{S P}=\frac{a}{\xi_{S P}^{\prime}}$ and rewrite $\boldsymbol{\Sigma}^{P O L}(\mathbf{k})$ as :

$$
\boldsymbol{\Sigma}^{P O L}(\mathbf{k})=-\frac{9}{\pi} \eta_{0} \frac{\Phi^{S P}}{\mathrm{~N}_{S}} t^{3}\left[1+W_{1}^{S P}\left(\Phi^{S P}\right)\right] \mathrm{Q}\left(t x_{S P}\right) k^{2} \mathbb{1}
$$

Both $x_{S P}\left(\Phi^{S P}\right)$ and $W_{1}^{S P}\left(\Phi^{S P}\right)$ are characteristic to the problem of the spheres immersed in a fluid and can be calculated from the system (48).

In a similar manner, the diffusion coefficient of the polymer chain in the effective medium of spheres can be expressed as :

$$
D\left(\Phi^{S P}\right)=\frac{\zeta_{t}^{K R}}{\zeta_{t}^{P O L}\left(\Phi^{S P}\right)}=\frac{3 \sqrt{\pi}}{4} \frac{1}{1+W_{1}^{S P}} \frac{\mathrm{P}\left(t x_{S P}\right)}{t x_{S P}}
$$

in which we used (C24), with $W_{1}^{P O L} \rightarrow W_{1}^{S P}$ and $\beta \rightarrow \beta_{S P}$. The function $\mathrm{P}(x)$ is defined in Appendix $\mathrm{C}$ (C23).
The effective viscosities, total and relative (which measures the increase in the viscosity due to the polymer chain) are calculated from :

$$
\begin{aligned}
\frac{\eta^{\mathrm{eff}}-\eta_{0}}{\eta_{0}} & =W_{1}^{S P}-\lim _{k \rightarrow 0} \frac{1}{\eta_{0}} \frac{\partial}{\partial k^{2}} \Sigma_{\perp}^{P O L}(\mathbf{k}) \\
& =W_{1}^{S P}+\frac{9}{\pi} \frac{\Phi^{S P}}{\mathrm{~N}_{S}} t^{3}\left(1+W_{1}^{S P}\right) \mathrm{Q}\left(t x_{S P}\right) \\
\frac{\eta^{\mathrm{eff}}-\eta_{\mathrm{SP}}}{\eta_{\mathrm{SP}}} & =-\lim _{k \rightarrow 0} \frac{1}{\eta_{\mathrm{SP}}} \frac{\partial}{\partial k^{2}} \Sigma_{\perp}^{P O L}(\mathbf{k}) \\
& =\frac{9}{\pi} \frac{\Phi^{S P}}{\mathrm{~N}_{S}} t^{3} \mathrm{Q}\left(t x_{S P}\right)
\end{aligned}
$$

with $\eta_{\mathrm{SP}}=\eta_{0}\left(1+W_{1}^{S P}\right)$, and $x_{S P}\left(\Phi^{S P}\right), W_{1}^{S P}\left(\Phi^{S P}\right)$ obtained from (48), as already mentioned.

As will be shown below, Eqs. 75,76 ) are the limiting formulas of the general situation presented in Section IVD, where the dependence of $D$ and $\eta^{\text {eff }}$ on $\Phi^{S P}$ and $t$ will be discussed also.

However, some remarks can be made. The arrest of the chain motion occurs at $\Phi_{D I V}^{S P} \simeq 0.49$-as found in Ref. [15] for a sphere moving in a random array of fixed spheres. The curves seem physically plausible. When the radius of gyration $R_{g}$ of the polymer chain is smaller than or comparable to the radius of the sphere $(t \lesssim 1)$ the viscosities (both total and relative) are relatively insensitive to variations in the $t$ parameter. Still, although being related only to the presence of the polymer chain, the relative viscosity depends on $\Phi^{S P}$ and $t$ variables through $\mathrm{Q}\left(t x_{S P}\right)$ (see 76$)$, so the contribution of the chain to the viscosity of the solution is a function of the effective solvent.

\section{One mobile sphere in a suspension of fixed spheres and polymers}

We now investigate the stationary dynamics of a suspension containing $\mathrm{N}_{S}$ uniformly distributed, rigid spheres of radius $a$ and $\mathrm{N}_{P}$ Gaussian, free moving polymer chains of length $L$. All spheres but one that is moving with the constant velocity $\mathbf{u}_{0}$, thus creating the flow, are fixed, and no interactions beside the hydrodynamic coupling occur. We calculate the diffusion coefficient $D$ of the moving sphere and the total $\eta^{\text {eff }}$ and the relative $\frac{\eta^{\text {eff }}}{\eta_{\mathrm{POL}}}-1$ effective viscosities as functions of the volume fractions $\Phi^{S P}$ for spheres, $\Phi^{P O L}$ for polymers and of the coupling parameter $t=\frac{R_{g}}{a}$.

We proceed closely to the derivations exposed in the previous sections. First, by properly identifying the $\mathcal{G}(\mathbf{k})$ tensor, we construct an effective medium replacing the polymers and the pure fluid,in this way reducing the problem to that of a self-consistent computation of 
the viscosity and diffusion coefficient of an ensemble of spheres, as discussed in Refs. [15, 5, 7] and in Sec. IIIA. Solving the resulting equations, we obtain the desired quantities,namely the diffusion coefficient of the moving sphere and the effective viscosities.

The velocity field obeys the N-S Eq. (27), with $\mathbf{F}(\mathbf{r}) \rightarrow$ $\mathbf{F}_{0}(\mathbf{r})$ now being the force exerted by the moving sphere upon the fluid. Averaging first over the distribution of the polymers and then over the position of the fixed spheres and introducing the self-energies $\mathbf{W}^{P O L}\left(\mathbf{r}, \mathbf{r}^{\prime}\right)$ and $\boldsymbol{\Sigma}^{S P}\left(\mathbf{r}, \mathbf{r}^{\prime}\right)$ as defined in (34), we deduce the N-S equation for the averaged velocity $\mathbf{u}(\mathbf{r})=\langle\mathbf{v}(\mathbf{r})\rangle$ :

$$
-\eta_{0} \triangle \mathbf{u}(\mathbf{r})+\langle\nabla p(\mathbf{r})\rangle-\mathbf{W}^{P O L} * \mathbf{u}-\boldsymbol{\Sigma}^{S P} * \mathbf{u}=\mathbf{F}_{0}(\mathbf{r})
$$

As usual, we denote the self-energy of the fluid resulting from the presence of the polymers with the $\mathbf{W}^{P O L}$ symbol and not with $\boldsymbol{\Sigma}^{P O L}$ to stress that this is the background effective medium we have related our calculations to. This also means that $\mathbf{W}^{P O L}$ depends only upon the polymer properties, but $\boldsymbol{\Sigma}^{S P}$ - the contribution of the spheres to the self-energy of the fluid - could depend on $\Phi^{P O L}$ and on some coupling parameter,like $t$ and,implicitly,on other characteristics of the background effective fluid. For $\mathbf{W}^{P O L}$ we will use the explicit expressions found in Sec. III B (Eqs. 55,56).

The formal solution of the N-S equation is then :

$$
\mathbf{u}(\mathbf{r})=\left.\mathcal{G} * \mathbf{F}\right|_{\mathbf{r}}
$$

with the modified Oseen tensor having the following form :

$$
\mathcal{G}(\mathbf{k})=\frac{\mathbb{1}-\hat{\mathbf{k}} \hat{\mathbf{k}}}{\eta_{0} k^{2}-W_{\perp}^{P O L}(\mathbf{k})-\Sigma_{\perp}^{S P}(\mathbf{k})}
$$

Working in the $k \rightarrow 0$ limit, we approximate $\boldsymbol{\Sigma}^{S P}(\mathbf{k})$ with :

$$
\boldsymbol{\Sigma}^{S P}(\mathbf{k})=\left(-\eta_{0} \xi_{S P}^{-2}-\eta_{0} \Sigma_{1}^{S P} k^{2}\right) \mathbb{1}
$$

where $\xi_{S P}$ is the screening length felt by the spheres in the effective medium that supplants for the polymers and the pure fluid. Substituting back in the Oseen tensor formula and replacing $W_{\perp}^{P O L}$ with its expression from $(53,56)$ we get :

$$
\mathcal{G}(\mathbf{k})=\frac{\mathbb{1}-\hat{\mathbf{k}} \hat{\mathbf{k}}}{\eta_{0}\left(1+\Sigma_{1}\right)\left(k^{2}+\xi^{\prime-2}\right)}=\frac{\mathbb{1}-\hat{\mathbf{k}} \hat{\mathbf{k}}}{\eta^{\mathrm{eff}}\left(k^{2}+\xi^{\prime-2}\right)}
$$

in which :

$$
\begin{aligned}
\Sigma_{1} & =W_{1}^{P O L}+\Sigma_{1}^{S P} \\
\xi^{\prime-2} & =\frac{\xi_{S P}^{-2}}{1+\Sigma_{1}}
\end{aligned}
$$

where $\xi^{\prime}$ is the total screening length in the resulting effective medium that replaced the initial pure fluid, the polymers and the spheres. Note that the polymers do not contribute to the screening, as found in Sec. III B.

It follows then that the total and the relative (due to the presence of spheres only) effective viscosities of the solution and the translational friction coefficient of the moving sphere (see Sec. IIIA) can be computed from :

$$
\begin{aligned}
\frac{\eta^{\mathrm{eff}}}{\eta_{0}}-1 & =W_{1}^{P O L}+\Sigma_{1}^{S P} \\
\frac{\eta^{\mathrm{eff}}}{\eta_{\mathrm{POL}}}-1 & =-\lim _{k \rightarrow 0} \frac{1}{\eta_{\mathrm{POL}}} \frac{\partial}{\partial k^{2}} \Sigma_{\perp}^{S P}(\mathbf{k})=\frac{1}{1+W_{1}^{P O L}} \Sigma_{1}^{S P} \\
\zeta_{t} & =-\frac{1}{c^{S P}} \Sigma_{\perp}^{S P}(k=0)=\eta_{0} \frac{1}{c^{S P}} \xi_{S P}^{-2}
\end{aligned}
$$

Next step is to find $\boldsymbol{\Sigma}^{S P}(\mathbf{k})$ self-consistently. This can be done exactly as shown in Appendix B,but taking care of the effective medium that replaces the polymers, by using the new $\mathcal{G}$, substituting $\Sigma_{1}$ for $W_{1}^{S P}$ and changing the meaning of $\xi^{\prime}$, which will now represent the total screening length. Thus :

$$
\begin{aligned}
& \boldsymbol{\Sigma}^{S P}(\mathbf{k})=-6 \pi a \eta_{0} c^{S P}\left(1+\Sigma_{1}\right) \frac{1}{\mathrm{I}_{1 / 2}\left(\frac{a}{\xi^{\prime}}\right) \mathrm{K}_{1 / 2}\left(\frac{a}{\xi^{\prime}}\right)} \mathbb{1} \\
& +\frac{3}{2} \eta_{0}\left(1+\Sigma_{1}\right) \Phi^{S P} k^{2} \mathrm{Z}\left(\frac{a}{\xi^{\prime}}\right) \mathbb{1}+\text { (longit. part) } \hat{\mathbf{z}} \hat{\mathbf{z}} \\
& \mathrm{Z}(y)=\frac{1}{\mathrm{I}_{1 / 2}(y) \mathrm{K}_{1 / 2}(y)}-\frac{8}{9 \mathrm{I}_{3 / 2}(y) \mathrm{K}_{3 / 2}(y)}-\frac{2}{5 \mathrm{I}_{1 / 2}(y) \mathrm{K}_{5 / 2}(y)}
\end{aligned}
$$

Comparing this expression with (80) and using the definitions of $\Sigma_{1}$ and $\xi^{\prime}$ from (82) and of $W_{1}^{P O L}$ from (56), we finally obtain the system of equations for $\boldsymbol{\Sigma}^{S P}(\mathbf{k})$ :

$$
\begin{aligned}
\Sigma_{1}^{S P}\left(\Phi^{S P}, \Phi^{P O L}\right) & =\frac{1}{1-\frac{18}{\pi \sqrt{\pi}} \Phi^{P O L}}\left[\frac{1}{1+\frac{3}{2} \Phi^{S P} \mathrm{Z}(x)}-1\right] \\
x^{2} & =\frac{9}{2} \Phi^{S P} \frac{1}{\mathrm{I}_{1 / 2}(x) \mathrm{K}_{1 / 2}(x)}
\end{aligned}
$$

with $x=x\left(\Phi^{S P}\right)=\frac{a}{\xi^{\prime}}$ and the function $\mathrm{Z}(x)$ being defined in the previous equation. One could remark that there is no polymer dependence in the total screening length $\xi^{\prime}$.

Solving this system for $x\left(\Phi^{S P}\right)$ and $\Sigma_{1}^{S P}\left(\Phi^{S P}, \Phi^{P O L}\right)$ allows us to compute the diffusion coefficient of the moving sphere from (83) :

$$
\begin{aligned}
D\left(\Phi^{S P}, \Phi^{P O L}\right)= & \frac{6 \pi a \eta_{0}}{\zeta_{t}}=\mathrm{I}_{1 / 2}(x) \mathrm{K}_{1 / 2}(x) \\
& \times\left(1-\frac{18}{\pi \sqrt{\pi}} \Phi^{P O L}\right)\left(1+\frac{3}{2} \Phi^{S P} \mathrm{Z}(x)\right)
\end{aligned}
$$


and the total and relative effective viscosities as :

$$
\begin{gathered}
\frac{\eta^{\mathrm{eff}}}{\eta_{0}}-1=\frac{1}{\left(1-\frac{18}{\pi \sqrt{\pi}} \Phi^{P O L}\right)\left[1+\frac{3}{2} \Phi^{S P Z}(x)\right]}-1 \\
\frac{\eta^{\mathrm{eff}}}{\eta_{\mathrm{POL}}}-1=\frac{1}{1+W_{1}^{P O L}} \Sigma_{1}^{S P}=\frac{1}{1+\frac{3}{2} \Phi^{S P Z}(x)}-1
\end{gathered}
$$

In Figs. 2, 2, 3 ; 此; 6 the results for $D$, the total $\eta^{\text {eff }} / \eta_{0}-1$ and the relative $\eta^{\text {eff }} / \eta_{\mathrm{POL}}-1$ viscosities have been plotted against $\Phi^{P O L}$ (first plot of each pair and Fig. 6) and against $\Phi^{S P}$ (in the second plot).

We have already seen that the total screening length $\xi^{\prime}$ as given by (85) is function only on $\Phi^{S P}$ (as the polymers were absent). It is also notable that there is no dependence upon the coupling parameter $t=\frac{R_{g}}{a}$. Both observations are related to the absence of screening in a dispersion of non-interacting polymers (as found in Sec. III B), which prevents the spheres to sense the existence of the polymer chains.

Two important points, at $\Phi_{D I V}^{S P} \simeq 0.49$ and at $\Phi_{D I V}^{P O L} \simeq$ 0.31 , where the viscosity of the solution and the friction coefficient diverge, are evident in Figs. 2 6. Note that $\Phi_{D I V}^{S P}+\Phi_{D I V}^{P O L}<1$, even if the polymer chains and the spheres are penetrable, non-interacting objects.

At low $\Phi^{S P}$ and $\Phi^{P O L} \rightarrow 0$ one recovers the Einstein result for the viscosity of spheres in a pure fluid and the Stokes friction coefficient for a sphere $\left(D\left(\Phi^{S P}, 0\right) \rightarrow 1\right)$.

When $\Phi^{S P} \rightarrow 0\left(\right.$ recall $\Phi^{S P}=\frac{\mathrm{N}_{S}-1}{V}$ ), we retrieve the results from the last section for one mobile sphere immersed in a polymer solution (compare Eqs. (36,87) to Eqs. (67,650). The results of this limit are marked with the + symbol in Figs. 2 and 1 .

\section{D. $\mathbf{N}_{P}$ polymer chains moving in a suspension of $\mathbf{N}_{S}$ randomly distributed fixed spheres}

In this section we explore the reverse of the previous model. There are $\mathrm{N}_{P}$ non-interacting, free-moving polymer chains of length $L$ dispersed in a suspension containing a random array of $\mathrm{N}_{S}$ rigid, penetrable, fixed spheres. We wish to compute - using the effective medium approach - the translational diffusion coefficient $D$ for a polymer chain and the total and relative (the spheres contribution) effective viscosities as function of $\Phi^{P O L}, \Phi^{S P}$ and of the coupling parameter $t=R_{g} / a$. The velocity flow is externally generated (one could consider it to be produced by the motion of one polymer chain without any influence on the final results).

Once again, we develop the necessary theory in two stages. First, we substitute the spheres and the pure fluid with the corresponding effective medium presented in Sec. III A, then we calculate self-consistenty the transport properties of the polymers dispersed in the effective solution, for any volume fraction $\Phi^{P O L}$, following the procedure detailed in Sec. IIIB. Conceptually, we are modeling this system with a superposition of two effective media, a fixed background related to the influence of the spheres and a foreground related to the polymers, coupled through hydrodynamic interactions.

Apparently, there should be a symmetry between the present system and the reversed one (spheres in a solution of polymers), but this assumption proves to be incorrect, as we will further show. The main difference is that in the present case the size coupling is active and $t=\frac{R_{g}}{a}$ controls both the mobility $D$ of the chains and the viscosity of the dispersion.

We start with the Navier-Stokes (N-S) equation (27) for the velocity field $\mathbf{v}(\mathbf{r})$ in the suspension, with $\mathbf{F}(\mathbf{r})$ some external force density. After we perform the configurational average (32) and we replace the averages over the forces with the $\mathbf{W}^{S P}\left(\mathbf{r}, \mathbf{r}^{\prime}\right)$ and $\boldsymbol{\Sigma}^{P O L}\left(\mathbf{r}, \mathbf{r}^{\prime}\right)$ as defined in (34) the N-S equation for the average velocity field $\mathbf{u}(\mathbf{r})$ is :

$$
-\eta_{0} \triangle \mathbf{u}(\mathbf{r})+\langle p(\mathbf{r})\rangle-\mathbf{W}^{S P} * \mathbf{u}-\boldsymbol{\Sigma}^{P O L} * \mathbf{u}=\mathbf{F}(\mathbf{r})
$$

where, as discussed in the previous sections, we substitute $\mathbf{W}^{S P}$ for $\boldsymbol{\Sigma}^{S P}$ to distinguish between the background effective fluid associated with the spheres and the contribution to the self-energy of the added polymer chains. $\mathbf{W}^{S P}\left(\mathbf{r}, \mathbf{r}^{\prime}\right)$ tensor is function only of $\Phi^{S P}$ volume fraction and it was calculated in Sec. IIIA, Eqs. (47, 48 ).

To obtain the self-consistent equations for $\boldsymbol{\Sigma}^{P O L}$ we proceed as in the last section. First, we write the formal solution of the N-S equation as a convolution :

$$
\mathbf{u}(\mathbf{r})=\mathcal{G} * \mathbf{F}
$$

where the modified Oseen tensor has its Fourier transform given by :

$$
\begin{aligned}
\mathcal{G}(\mathbf{k}) & =\frac{\mathbb{1}-\hat{\mathbf{k}} \hat{\mathbf{k}}}{\eta_{0} k^{2}-W_{\perp}^{S P}(\mathbf{k})-\Sigma_{\perp}^{P O L}(\mathbf{k})} \\
& =\frac{\mathbb{1}-\hat{\mathbf{k}} \hat{\mathbf{k}}}{\eta_{0}\left(1+\Sigma_{1}\right)\left(k^{2}+\xi^{\prime-2}\right)} \\
\Sigma_{1} & =W_{1}^{S P}+\Sigma_{1}^{P O L} ; \xi^{\prime-2}=\frac{\xi_{S P}^{-2}+\xi_{P O L}^{-2}}{1+\Sigma_{1}}
\end{aligned}
$$

in which we inserted $W_{\perp}^{S P}$ from (45) and we used the following approximation, in the $k \rightarrow 0$ region :

$$
\boldsymbol{\Sigma}^{P O L}(\mathbf{k})=\left(-\eta_{0} \xi_{P O L}^{-2}-\eta_{0} \Sigma_{1}^{P O L} k^{2}\right) \mathbb{1}
$$

Also, $\xi^{\prime}$ is the total screening length and $\xi_{S P}$ is function only of the concentration of spheres. We can readily write 
the solution for $\boldsymbol{\Sigma}^{P O L}(\mathbf{k})$ by observing the structures of the Oseen tensors found in (52) and (90). In comparison with the effective medium theory for polymers (as in Sec. III B and Appendix C), we need to transform (55) by working with $\xi^{\prime}$ instead of $\xi_{S P}^{\prime}$ and with the total self-energy $\Sigma_{1}$ instead of $W_{1}^{P O L}$. We immediately arrive to :

$$
\boldsymbol{\Sigma}^{P O L}(\mathbf{k})=-\frac{9}{\pi} \eta_{0} \Phi^{P O L}\left(1+\Sigma_{1}\right) \mathrm{Q}(\beta) k^{2} \mathbb{1}
$$

with $\beta=\frac{R_{g}}{\xi^{\prime}}$ and the function $\mathrm{Q}(\mathrm{x})$ defined by 55 . Comparing to (90), we obtain the system :

$$
\begin{aligned}
\xi_{P O L}^{-2} & =0 \\
\Sigma_{1}^{P O L} & =\frac{9}{\pi} \Phi^{P O L}\left(1+W_{1}^{S P}+\Sigma_{1}^{P O L}\right) \mathrm{Q}(\beta)
\end{aligned}
$$

Introducing the natural dimensionless variables $x\left(\Phi^{S P}\right)=\frac{a}{\xi_{S P}^{\prime}}$ and $t=\frac{R_{g}}{a}$, we finally determine $\boldsymbol{\Sigma}^{P O L}(\mathbf{k})$ through :

$$
\begin{aligned}
\Sigma_{1}^{P O L}\left(\Phi^{P O L}, \Phi^{S P}, t\right)= & {\left[1+W_{1}^{S P}\left(\Phi^{S P}\right)\right] } \\
& \times \frac{1}{1-\frac{9}{\pi} \Phi^{P O L} \mathrm{Q}(\beta)} \\
\beta^{2}= & (t x)^{2}\left(1-\frac{9}{\pi} \Phi^{P O L} \mathrm{Q}(\beta)\right)
\end{aligned}
$$

where $W_{1}^{S P}$ and $x\left(\Phi^{S P}\right)$ are computed from the system (48) as functions of $\Phi^{S P}$ only. Note that now $\beta\left(=R_{g} / \xi^{\prime}\right)$ is in general a non-zero function of $\Phi^{P O L}, \Phi^{S P}$ and $t$, which means that the polymers are subjected to a screened hydrodynamic interaction, in contrast with the previous system.

The effective viscosities - total and relative, due to added polymers - are determined from :

$$
\begin{aligned}
\frac{\eta^{\mathrm{eff}}}{\eta_{0}}-1 & =W_{1}^{S P}+\Sigma_{1}^{P O L} \\
& =\left(1+W_{1}^{S P}\right) \frac{1}{1-\frac{9}{\pi} \Phi^{P O L} \mathrm{Q}(\beta)}-1 \\
\frac{\eta^{\mathrm{eff}}}{\eta_{\mathrm{SP}}}-1 & =\frac{1}{1+W_{1}^{S P}} \Sigma_{1}^{P O L}=\frac{1}{1-\frac{9}{\pi} \Phi^{P O L} \mathrm{Q}(\beta)}-1
\end{aligned}
$$

For a mobile polymer chain the translational diffusion coefficient (actually defined as relative to the KirkwoodRiseman result) is shown to be (see Appendix C) :

$$
D=\frac{\zeta_{t}^{K R}}{\mathcal{G}_{00}^{-1}}
$$

with $\mathcal{G}_{00}^{-1}$ being the zero-th coefficient of a Fourier series expansion of $\left\langle\mathcal{G}^{-1}\right\rangle$ (see Appendix C). Because in our approximations $\mathcal{G}_{q q^{\prime}}$ is diagonal, $\mathcal{G}_{00}^{-1}$ becomes C23 :

$$
\mathcal{G}_{00}^{-1}=\left(\mathcal{G}_{0}\right)^{-1}=3 \pi \eta_{0} R_{g} \frac{\beta}{\mathrm{P}(\beta)}\left(1+\Sigma_{1}\right)
$$

The diffusion coefficient of any moving polymer chain is given by :

$$
D=\frac{3 \sqrt{\pi}}{4} \frac{1}{1+W_{1}^{S P}} \frac{\mathrm{P}(\beta)}{\beta}\left(1-\frac{9}{\pi} \Phi^{P O L} \mathrm{Q}(\beta)\right)
$$

The functions $\mathrm{P}(x)$ and $\mathrm{Q}(x)$ have been defined in Appendix C, Eqs. (C23, C28).

The prominent feature of the final results for the solution viscosity and the diffusion coefficient of a polymer chain $(94,97)$ is their dependence on the $t$ parameterthe stationary dynamics of the spheres and the polymer chains is size coupled - as advertised in the beginning. Also note that in contrast to the problem of spheres in a polymer suspension, the variable $\beta=R_{g} / \xi^{\prime}$ depends upon $\Phi^{S P}$ too, which implies - Eq. (94) - that the relative contribution of the polymers to the viscosity is enhanced, function of $\Phi^{S P}$ and $t$, by the presence of the background effective fluid related to the spheres. It is sensible to believe that these new phenomena have been generated by the spheres acting as fixed scattering centers of the velocity field and had we analyzed a system of polymer chains and Brownian spheres, we might have observed that the dynamics of the two types of particles would have been decoupled, in the mentioned sense (no $t$ dependence, $\beta$ function only on $\left.\Phi^{P O L}\right)$.

Checking the limit $\Phi^{S P} \rightarrow 0$ we recover the results for the suspension of polymers only $\left(W_{1}^{S P} \rightarrow 0, \beta \rightarrow 0\right.$, compare to (57)), and in the limit $\Phi^{P O L} \rightarrow 0$ we retrieve the results for the $\mathbf{1 P} / \mathbf{S}$ problem : $\beta \rightarrow t x_{S P}$; $\Phi^{P O L} \rightarrow \frac{1}{V} 4 \pi R_{g}^{3} / 3=\frac{\Phi^{S P}}{\mathrm{~N}_{S}} t^{3}$ (compare to Eqs. (75, , 76) ).

The values of the total and relative effective viscosities and of the diffusion coefficient given by Eqs. (94, 97) have been plotted as functions of $\Phi^{P O L}$ and $\Phi^{S P}$ at different $t$. Four sets of data corresponding to $t=\{0.01 ; 0.1 ; 1 ; 10\}$ have been calculated and displayed.

In Figs. $[7]$ 10] the diffusion coefficient $D$ of a moving polymer chain is plotted against $\Phi^{S P}$ with $\Phi^{P O L}$ parameter and for each of the $t$ values mentioned, and in Figs. 12110 is plotted as a function of $\Phi^{P O L}$ with $\Phi^{S P}$ parameter, with $t=\{0.1,1,10\}$ for convenience.

$D\left(\Phi^{P O L}\right)$ depends linearly on $\Phi^{P O L}$ and for fixed $\Phi^{S P}$ and $\Phi^{P O L}$,the diffusion coefficient $D$ decreases as $t$ increases - longer chains are less diffusive - so the $t$ parameter acts like a mobility selector. The sensitivity of $D$ to $t$ variations is higher when the size $\left(R_{g}\right)$ of the chains becomes comparable to the radius of the spheres (compare the relative drop in $D$ at the same $\Phi^{S P}$ in Fig. 12 $(t=0.1)$ to Fig. $13(t=1)$, and Fig. 13 to Fig. 14 $(t=10)$.

The same observation can be made when looking at the $\Phi^{S P}$ dependence of $D$, in Figs. [7 10], for the same 
values of $t$. Additionally, for $t=\{0.01 ; 0.1\}, D$ exhibits a change in the curvature and an almost plateau region in the middle of the $\Phi^{S P}$ range for higher $\Phi^{P O L}$ (Figs. 7, [8).

We now consider the behavior of the limiting case corresponding to the $\mathbf{1 P} / \mathbf{S}$ system as shown by the + symbols in Figs. [7 10]. One can notice a change in the curvature of the diffusion coefficient of one polymer chain when going from $t=0.01$ to $t=10$. It appears that for relatively small (in comparison to the spheres) polymer chains $(t=0.01)$ or relatively large $(t=10)$ a plateau region appears, where $D$ is slowly varying with respect to $\Phi^{S P}$. Physically, this could signify that when the polymer is small (large), a certain built up in $\Phi^{S P}$ is required before the diffusion coefficient $D$ is affected more markedly. Additionally, for a given radius $a$ of the spheres,the difference in the mobilities of two polymer chains of distinct lengths $\left(t_{1} \neq t_{2} ; t_{i}=\frac{R_{g}^{(i)}}{a}\right)$ is maximized for a certain range of $\Phi^{S P}$ values, fact depicted in Fig. 11, where the difference in the diffusion coefficient of one polymer chain for four pairs of $t$ values is plotted against the filling fraction $\Phi^{S P}$ (e.g. $\Phi^{S P} \in[.15 ; .3]$ roughly for two types of polymer chains with $t_{1}=0.01$ and $t_{2}=1$ ). This may have applications in the design of polymer sieves.

Comparing now the behavior of the polymer moving in the suspension of spheres (Figs. [7] 10]) to that of the mobile sphere inside a polymer dispersion (Fig. 2) at equivalent relative sizes of the tracer particle $(t=10$ in the first case is similar to $t=0.1$ in the second one), we notice that the $D\left(\Phi^{S P}\right)$ for a small mobile polymer chain $(t=0.01 ; 0.1)$ is higher than that of a small sphere $(t=10)$ moving in a polymer suspension, but a larger sphere $(t=0.1)$ exhibits an increased mobility $(D)$ comparative to a large polymer chain $(t=1 ; 10)$. Eventually, because $\left(\Phi_{D I V}^{S P} \simeq 0.49\right)>\Phi_{D I V}^{P O L} \simeq 0.31$, the polymer immersed among spheres will retain a non-zero mobility at higher volume fractions than the sphere moving in a polymer suspension.

The same discussion applies in the general $\mathbf{P} / \mathbf{S}$ case. The mobility of a small $t=0.01$ or large $t=10$ polymer chain is only slightly influenced by the volume fraction of the spheres (see slopes of graphs in Figs. 7,10 in the middle region). This feature indicates that, for a given radius $a$ of the background spheres and a pair of polymer chains characterized by distinct $t$ values (e.g. $t_{1}=0.01$ and $t_{2}=1$ ), the difference in the diffusion coefficients of the two chains will reach its maximum for a certain range of the spheres volume fraction $\Phi^{S P}$.

The two points of divergence $\Phi_{D I V}^{P O L} \simeq 0.31$ and $\Phi_{D I V}^{S P} \simeq 0.49$ are clearly marked. Because $\Phi_{D I V}^{P O L}<\Phi_{D I V}^{S P}$ the suppressing of the mobility of the moving particles (either sphere or a mobile polymer chain) is stronger when polymers are added than when the sphere concentration is increased.

Comparing the data for $D\left(\Phi^{S P}\right)$ in Fig. B, for the $\mathbf{S} / \mathbf{P}$ example (note that there is no $t$ dependence, to those for $D\left(\Phi^{P O L}\right)$ at $t=0.1$ in Fig. 12 and $t=10$ in Fig. 14, the same observation as in the $\mathbf{1 P} / \mathbf{S}$ case can be made : at the same $t$ ratio between the sizes of the moving object and of the background component and for the same background concentration, a small $(t=0.1)$ polymer chain in the $\mathbf{P} / \mathbf{S}$ system is more mobile than a small $(t=10)$ sphere moving in the $\mathbf{S} / \mathbf{P}$ system, but as $t$ gets larger, a big sphere becomes more diffusive than a long polymer chain $(t=10)$. In this situation, the control parameter is the concentration of the added (e.g., in the $\mathbf{P} / \mathbf{S}$ example, polymers are the added elements) components, but the same conclusion is reached if comparing the data for $D\left(\Phi^{P O L}\right)$ from Fig. 2 in the $\mathbf{S} / \mathbf{P}$ case to those from Fig. $8(t=0.1)$ and Fig. $10(t=10)$ for the $\mathbf{P} / \mathbf{S}$ system, the control parameter now being the concentration of the background elements.

The limiting case of one polymer chain $\left(\mathrm{N}_{P} \rightarrow 1\right)$ is very well approached in the $\mathbf{P} / \mathbf{S}$ example, as one can notice in Figs. 710 by comparing the uppermost curves $(\mathbf{1 P} / \mathbf{S}$ case $)$ to those corresponding to the parameter value $\Phi^{P O L}=0.01$, representing $D\left(\Phi^{S P}\right)$ when $t=\{0.01 ; 0.1 ; 1 ; 10\}$, respectively.

Viscosities calculated from (94) have been plotted in Figs. 15 21 : the total effective viscosity change in Figs. 15.16 as a function of $\Phi^{P O L}$ and against $\Phi^{S P}$ in Figs. 17. 18 with $t=\{0.01 ; 10\}$; the relative effective viscosity in Fig. 19 as a function of $\Phi^{P O L}$ for $t=\{1 ; 10\}$ and as a function of $\Phi^{S P}$ in Figs. 20, 21 with $t=\{0.01 ; 1 ; 10\}$. Two observations could be made regarding the viscosities :

a) both for $\eta^{\mathrm{eff}}\left(\Phi^{P O L}\right)$ with $\Phi^{S P}$ parameter (Figs. 15,16) and for $\eta^{\text {eff }}\left(\Phi^{S P}\right)$ with $\Phi^{P O L}$ parameter (Figs. 17, 18), as $t$ increases, the total viscosity change is more sensitive to variations in $\Phi^{P O L}$ or $\Phi^{S P}$ (compare Fig. 15 when $t=0.01$ to Fig. 16 when $t=10$ and Fig. $17(t=0.01)$ to Fig. $18(t=10))$. We note that a larger polymer chain (in comparison to the radius of the background sphere) is more sensitive to the environment. This behavior clearly contrasts that encountered for the total effective viscosity in the $\mathbf{S} / \mathbf{P}$ case (see Fig. (1)).

b) the relative effective viscosity due to the presence of the polymers (Figs. 19 21) depends not only on $\Phi^{P O L}$ but on $\Phi^{S P}$ and on $t$ as well, once again in contrast to the $\mathbf{S} / \mathbf{P}$ system (see Fig. 6). As suggested before, $t$ acts like a control parameter that when increased (compare $t=1$ to $t=10$ calculations in Fig. 19 or Fig. 20 to Fig. 21) makes the contribution of the polymers to the viscosity more markedly dependent on both $\Phi^{P O L}$ and $\Phi^{S P}$. Actually, when the radius of gyration $R_{g}$ is comparable to the sphere diameter $a(t \leq 1)$, the relative viscosity $\frac{\eta^{\text {eff }}}{\eta_{\mathrm{SP}}}-1$ is relatively insensitive to variations in $\Phi^{S P}$ or $t$ (compare Fig. 20 to $t=1$ data in Fig. 21).

Obviously, these observations may have some significance from an experimental point of view. 


\section{CONCLUSIONS}

In the present paper we have investigated the steadystate transport properties of a heterogeneous suspension of rigid polymers and spheres without excluded volume interactions, applying the multiple scattering formalism [4, 6, 18 and the effective medium theory [7, 15, 18].

The main approximations and features of our approach were :

a. the absence of any interactions among polymers and spheres. All particles are penetrable.

b. the solvent is assumed incompressible and it is described by a linearized Navier-Stokes equation.

c. the stationary, time-independent limit is assumed. All results are valid in the long range hydrodynamic limit $(k \rightarrow 0)$.

d. the dynamics of the solvent, polymers and of the spheres are coupled by stick boundary conditions.

e. the spheres are monodisperse, having radius $a$.

f. the polymers are monodisperse with chain length $L$, and they translate freely as rigid bodies. We do not consider the rotation of the polymer as a rigid body. For convenience, the distribution function for the inter-segment distance of any chain was taken to be Gaussian.

g. $\boldsymbol{\Sigma}(\mathbf{k})$, the self-energy of the fluid in the presence of the immersed spheres and polymer chains was approximated with the first-order component $\boldsymbol{\Sigma}^{(1)}(\mathbf{k})$, in an expansion in the number of distinct scattering events (one event means one interaction of the velocity field with one particle). When no correlations (interactions) among particles occur,this is actually the leading term of the exact $\boldsymbol{\Sigma}(\mathbf{k})$.

Under these approximations, two general systems have been analyzed :

1. Moving sphere in a solution of fixed spheres and freely translating polymer chains.

2. Moving polymer chains dispersed in a suspension of fixed spheres.

Also, two significant limiting cases of the previous models have been investigated :

a. Moving sphere in a solution of polymers.

b. Moving polymer chain immersed in an array of fixed spheres.
For each example the underlying theory has been exposed and developed and equations were explicitly displayed, from which the translational diffusion coefficient $D$ of the probe particle and the effective viscosities of the solution, total $\eta^{\text {eff }} / \eta_{0}-1$ and relative, have been computed.

We have identified the proper dimensionless variables characterizing the problem as follows : $\beta=\frac{R_{g}}{\xi^{\prime}}, x=\frac{a}{\xi^{\prime}}$ and $t=\frac{R_{g}}{a}$, where $R_{g}$ and $a$ are the radius of gyration of a polymer chain and the radius of a sphere, and $\xi^{\prime}$ is the total screening length in the solution.

It has been shown that the polymers do not contribute to the screening of the hydrodynamic interactions at large length scales and, as functions of polymer filling fraction $\Phi^{P O L}$, both the effective viscosity $\eta^{\text {eff }}$ and the translational friction coefficient $\zeta_{t}$ diverge, in all examples, at a volume fraction $\Phi_{D I V}^{P O L} \simeq 0.31$ much less than the corresponding divergence point for the $\Phi^{S P}$ dependence $\left(\Phi_{D I V}^{S P} \simeq 0.49\right)$. While for suspensions of uniform particles showing no long-range order - in particular spheres - there is wide experimental support for the divergence of viscosity and of the friction coefficient for $\Phi_{D I V}^{S P} \in[0.5,0.6]$ (e.g. Ref. [19]), in the case of solutions of rigid polymers and specifically for suspensions of polymers and spheres less data are available, to our knowledge.

Perhaps the most interesting parameter is $t$, which is controlling the response of $\eta^{\text {eff }}$ and $D$, both for spheres and polymers, to changes in the environment (variations in $\Phi^{S P}$ and $\left.\Phi^{P O L}\right)$. The understanding of the role of the $t$ variable might be of interest in experimental applications, mainly related to gel permeation chromatography and macromolecular sieving. A rather noticeable prediction of our analysis related to the process of diffusion of the polymers in a suspension of fixed spheres is the existence of an optimum range of the volume fraction $\Phi^{S P}$ of the spheres that maximizes the difference in the diffusion coefficients for two types of chains characterized by distinct $t$ values (see Fig. 11 for the $\mathbf{1 P} / \mathbf{S}$ system and Figs. 710 in the $\mathbf{P} / \mathbf{S}$ case).

It has been also noticed that at the same $t$ ratio between the size of the tracer (moving) particle and the size of the background element and the same background concentration, the spheres and the polymers are behaving differently. When the polymer is small in comparison to the spheres, it is more diffusive than a small sphere moving among polymer chains, but a large polymer chain in a suspension of fixed spheres is less mobile than a large sphere moving in a polymer dispersion. We must note that this observation is valid before reaching $\Phi_{D I V}^{P O L} \simeq 0.31$.

Despite the approximations made to yield the calculations analytically tractable, the formalism presented is essentially general and also applicable when excludedvolume (or other types of) interactions are turned on, although in practice the effort could be quite laborious. 
Yet, in this straightforward approach we have managed to draw a physically reasonable picture of the dynamical behavior of such heterogeneous solutions of polymers and spheres and some conceivable findings emerged. Further extensions of this work are possible, for example the study of rigid rodlike polymers and spheres, microemulsions (deformable particles) or investigating polydisperse suspensions of spheres and polymers. This last aspect is particularly meaningful in industrial applications (e.g. ceramic fabrication, where processing at volume fractions higher than the limiting values $\Phi_{D I V}^{S P}, \Phi_{D I V}^{P O L}$ is necessary but very difficult due to the divergent viscosity), because experimentally it has been shown that a proper adjustment of the particle size distribution could induce substantial increases in the limiting volume fractions [19], but theoretically this observations is still not fully understood.

We seek to address this question and the physically more appealing problem of a heterogeneous suspension of particles with potential interactions in a future paper.

\section{ACKNOWLEDGMENTS}

Acknowledgment is made to the Materials Research Science and Engineering Center at the University of Massachusetts, and the NSF Grant DMR 9221146001.

\section{APPENDIX A: SOLUTION OF THE NAVIER-STOKES EQUATION FOR A SUSPENSION OF $\mathrm{N}_{P}$ POLYMER CHAINS AND $\mathbf{N}_{S}$ SPHERES}

In Sec. IIB we have found that a formal solution of the Navier-Stokes equation (27) is given by Eq. (30), where the force densities $\boldsymbol{\sigma}_{\alpha i}$ and $\boldsymbol{\sigma}_{b}$ exerted upon the fluid by the $i$-th bead of the polymer chain $\alpha$ and by the point $\mathbf{R}_{b}$ on the surface of the sphere $b$, are unknowns. Using the stick boundary conditions (28), we eliminate the forces to express the velocity field in terms of the single-object flow propagators $\mathbf{T}_{\alpha}$ of any chain $\alpha$ and $\mathbf{T}_{b}$ of any sphere.

To simplify the notation, we replace the variables $\mathbf{R}_{b}, \mathbf{R}_{\alpha i}$ with the indices $b$ and $\alpha i$,respectively, when appearing in the arguments of the Oseen tensor $\mathbf{G}$ and we apply the Einstein convention of summation over repeating indices. In the case of the spheres this will also imply a surface integral over the solid angle $\mathrm{d} \Omega$. We drop the chain index $\alpha$ for relations generally valid independent of the chain variables. The convolution operation is denoted by $*-\mathbf{f} * \mathbf{g}=\int d \mathbf{r}^{\prime} \mathbf{f}\left(\mathbf{r}-\mathbf{r}^{\prime}\right) \cdot \mathbf{g}\left(\mathbf{r}^{\prime}\right)$ with $\mathbf{f}$ and $\mathbf{g}$ tensors or vectors - and it is understood that its result is a function of some position vector $\mathbf{r}$, when not otherwise specified by a subscript. Due to the translational invariance in the suspension, $\mathbf{G}\left(\mathbf{r}, \mathbf{r}^{\prime}\right)$ is actually a function of the relative distance : $\mathbf{G}\left(\mathbf{r}-\mathbf{r}^{\prime}\right)$. Then, Eq. (30) can be rewritten as :

$$
\mathbf{v}(\mathbf{r})=\mathbf{G} * \mathbf{F}+\mathbf{G}_{\mathbf{r}, \alpha i} \cdot \boldsymbol{\sigma}_{\alpha i}+\mathbf{G}_{\mathbf{r}, b} \cdot \boldsymbol{\sigma}_{b}
$$

The sums over $i, j, \ldots$ run from 1 to $n$, the sums over $\alpha, \beta, \ldots$ from 1 to $\mathrm{N}_{P}$ and the sums over $b, c, \ldots$ types of indices run from 1 to $\mathrm{N}_{S}$. Inserting the previous expression in the boundary conditions (28) we get :

$$
\begin{aligned}
\mathbf{u}_{\alpha}+\boldsymbol{\omega} \times \mathbf{S}_{\alpha k} & =\left.\mathbf{G} * \mathbf{F}\right|_{\alpha}+\mathbf{G}_{\alpha k, \beta j} \cdot \boldsymbol{\sigma}_{\beta j}+\mathbf{G}_{\alpha k, b} \cdot \boldsymbol{\sigma}_{b} \\
0 & =\left.\mathbf{G} * \mathbf{F}\right|_{b}+\mathbf{G}_{b, \alpha i} \cdot \boldsymbol{\sigma}_{\alpha i}+\mathbf{G}_{b, c} \cdot \boldsymbol{\sigma}_{c}
\end{aligned}
$$

Here $\mathbf{u}_{\alpha}$ and $\boldsymbol{\omega}_{\alpha}$ are the velocity of the center of mass and the angular velocity of chain $\alpha$. To eliminate the unknown quantities $\boldsymbol{\sigma}_{\alpha i}$ and $\boldsymbol{\sigma}_{b}$ we need the generalized inverse operators $\mathbf{K}_{\alpha}^{-1}$ and $\mathbf{K}_{b}^{-1}$ 12, 15 defined by :

$$
\begin{aligned}
& \sum_{j=1}^{n} \mathbf{K}_{\alpha}^{-1}\left(\mathbf{S}_{i}, \mathbf{S}_{j}\right) \cdot \mathbf{G}\left(\mathbf{S}_{\alpha j}-\mathbf{S}_{\alpha k}\right)=\mathbb{1} \delta_{\alpha i, \alpha k} \\
& \int d \Omega_{b}^{\prime \prime} \mathbf{K}_{b}^{-1}\left(\mathbf{r}, \mathbf{r}^{\prime \prime}\right) \cdot \mathbf{G}\left(\mathbf{r}^{\prime \prime}-\mathbf{r}^{\prime}\right)=\mathbb{1} \delta\left(\Omega_{b}-\Omega_{b}^{\prime}\right)
\end{aligned}
$$

where $\mathbf{r}\left(\Omega_{b}\right), \mathbf{r}^{\prime \prime}\left(\Omega_{b}^{\prime \prime}\right)$ and $\mathbf{r}\left(\Omega_{b}^{\prime}\right)$ are position vectors (about the center of mass frame) of distinct points on the surface of the sphere $b$ and $\mathbf{S}_{\alpha i}$ is the position vector of the $i$-th bead of the chain $\alpha$ with respect to its center of mass. Applying the inverse operators in (A2) we obtain :

$$
\begin{aligned}
& \boldsymbol{\sigma}_{\alpha i}=\sum_{k=1}^{n} \mathbf{K}_{\alpha i, \alpha k}^{-1} \cdot\left(\mathbf{u}_{\alpha}-\left.\mathbf{G} * \mathbf{F}\right|_{\alpha k}-\mathbf{G}_{\alpha k, b} \cdot \boldsymbol{\sigma}_{b}\right. \\
& \left.-\left.\mathbf{G}_{\alpha k, \beta j} \cdot \boldsymbol{\sigma}_{\beta j}\right|_{\beta \neq \alpha}\right)+\sum_{k=1}^{n} \mathbf{K}_{\alpha i, \alpha k}^{-1} \cdot\left(\boldsymbol{\omega}_{\alpha} \times \mathbf{S}_{\alpha k}\right) \\
& \boldsymbol{\sigma}_{b}\left(\Omega_{b}\right)=-\left.\mathbf{K}_{b b^{\prime}}^{-1} \cdot \mathbf{G} * \mathbf{F}\right|_{b^{\prime}}-\mathbf{K}_{b b^{\prime}}^{-1} \cdot \mathbf{G}_{b^{\prime}, \alpha i} \cdot \boldsymbol{\sigma}_{\alpha i} \\
& -\left.\mathbf{K}_{b b^{\prime}}^{-1} \cdot \mathbf{G}_{b^{\prime} c} \cdot \boldsymbol{\sigma}_{c}\right|_{b \neq c}
\end{aligned}
$$

Note that there is no summation over $\alpha$ in the first equation and over $b$ in the second one.

Analyzing the structure of these force factors, it appears manifest that the force upon the fluid due to one object, is proportional to the relative increase in the velocity of the fluid about the local velocity owing to the action of all other bodies and external forces, which is a sensible physical expectation. Also, the inverse operators $\mathbf{K}_{\alpha i, \alpha k}^{-1}$ and $\mathbf{K}_{b b^{\prime}}^{-1}$ will produce the friction coefficients of the polymer chains and of the mobile sphere, after one performs the configurational average (32) and sums over the indices $i, k$ in the polymer case or integrates over $\Omega_{b}$ and $\Omega_{b}^{\prime}$ for the sphere.

As shown in Ref. 12], the angular velocity of the chain $\boldsymbol{\omega}_{\alpha}$ in the force equations (A4) leads, eventually, to the appearance of the rotational and cross translationalrotational/rotational-translational friction coefficients of 
the polymer chains. The former has been calculated in Ref. [12] and the latter vanish upon preaveraging. In the present work we will ignore the rotational term, which does not alter the screening properties of a polymer solution. Then, using the conditions (29) that the total external force and torque acting on any polymer chain are zero, we get the velocity $\mathbf{u}_{\alpha}$ :

$$
\begin{gathered}
\mathbf{u}_{\alpha}=\left.\mathbf{g}_{t}^{-1} \cdot \sum_{i, k}^{n} \mathbf{K}_{i k}^{-1} \cdot \mathbf{G} * \mathbf{F}\right|_{i k}+\mathbf{g}_{t}^{-1} \cdot \sum_{i, k}^{n} \mathbf{K}_{\alpha i, \alpha k}^{-1} \\
\cdot \mathbf{G}_{\alpha k, b} \cdot \boldsymbol{\sigma}_{b}+\left.\mathbf{g}_{t}^{-1} \cdot \sum_{i, k}^{n} \mathbf{K}_{\alpha i, \alpha k}^{-1} \cdot \mathbf{G}_{\alpha k, \beta j} \cdot \boldsymbol{\sigma}_{\beta j}\right|_{\beta \neq \alpha} \\
\mathbf{g}_{t}=\sum_{i, j}^{n} \mathbf{K}_{i j}^{-1} ; \mathbf{g}_{t} \cdot \mathbf{g}_{t}^{-1}=\mathbb{1}
\end{gathered}
$$

Inserting back in (A4) and relabeling the dummy indices, we finally calculate :

$$
\begin{aligned}
\boldsymbol{\sigma}_{\alpha i}= & \left.\mathbf{T}_{\alpha i, \alpha k} \cdot \mathbf{G} * \mathbf{F}\right|_{\alpha k}+\left.\mathbf{T}_{\alpha i, \alpha k} \cdot \mathbf{G}_{\alpha k, \beta j} \cdot \boldsymbol{\sigma}_{\beta j}\right|_{\beta \neq \alpha} \\
& +\mathbf{T}_{\alpha i, \alpha k} \cdot \mathbf{G}_{\alpha k, b} \cdot \boldsymbol{\sigma}_{b} \\
\boldsymbol{\sigma}_{b}= & \left.\mathbf{T}_{b b^{\prime}} \cdot \mathbf{G} * \mathbf{F}\right|_{b^{\prime}}+\mathbf{T}_{b b^{\prime}} \cdot \mathbf{G}_{b^{\prime}, \alpha i} \cdot \boldsymbol{\sigma}_{\alpha i} \\
& +\left.\mathbf{T}_{b b^{\prime}} \cdot \mathbf{G}_{b^{\prime} c} \boldsymbol{\sigma}_{c}\right|_{b \neq c}
\end{aligned}
$$

where there is no summation over $\alpha$ in the first equation and over $b$ in the second and we have introduced the notation :

$$
\begin{aligned}
\mathbf{T}_{\alpha i, \alpha k} & =-\left[\mathbf{K}_{\alpha i, \alpha k}^{-1}-\sum_{l, l^{\prime}=1}^{n} \mathbf{K}_{\alpha i, \alpha l}^{-1} \cdot \mathbf{g}_{t}^{-1} \cdot \mathbf{K}_{\alpha l^{\prime}, \alpha k}^{-1}\right] \\
\mathbf{T}_{b b^{\prime}} & =-\mathbf{K}_{b b^{\prime}}^{-1}
\end{aligned}
$$

Following indefinite iterations in A6 and substituting the results for $\boldsymbol{\sigma}_{\alpha i}$ and $\boldsymbol{\sigma}_{b}$ back in (A1), we obtain the velocity field as :

$$
\begin{aligned}
\mathbf{v}(\mathbf{r}) & =\mathbf{G} * \mathbf{F}+\left.\mathbf{G}_{\mathbf{r} \alpha i} \cdot \mathbf{T}_{\alpha i, \alpha k} \cdot \mathbf{G} * \mathbf{F}\right|_{\alpha k} \\
& +\left.\mathbf{G}_{\mathbf{r} b} \cdot \mathbf{T}_{b b^{\prime}} \cdot \mathbf{G} * \mathbf{F}\right|_{b^{\prime}} \\
& +\left.\left.\mathbf{G}_{\mathbf{r} \alpha i} \cdot \mathbf{T}_{\alpha i, \alpha k} \cdot \mathbf{G}_{\alpha k, \beta j} \cdot \mathbf{T}_{\beta j, \beta l}\right|_{\beta \neq \alpha} \cdot \mathbf{G} * \mathbf{F}\right|_{\beta l} \\
& +\left.\mathbf{G}_{\mathbf{r} b} \cdot \mathbf{T}_{b b^{\prime}} \cdot \mathbf{G}_{b^{\prime} c} \cdot \mathbf{T}_{c c^{\prime}} \cdot \mathbf{G} * \mathbf{F}\right|_{b \neq c} \\
& +\left.\mathbf{G}_{\mathbf{r}, \alpha i} \cdot \mathbf{T}_{\alpha i, \alpha k} \cdot \mathbf{G}_{\alpha k, b} \cdot \mathbf{T}_{b b^{\prime}} \cdot \mathbf{G} * \mathbf{F}\right|_{b^{\prime}} \\
& +\left.\mathbf{G}_{\mathbf{r} b} \cdot \mathbf{T}_{b b^{\prime}} \cdot \mathbf{G}_{b^{\prime}, \alpha i} \cdot \mathbf{T}_{\alpha i, \alpha k} \cdot \mathbf{G} * \mathbf{F}\right|_{\alpha k}+\ldots
\end{aligned}
$$

Next, the single chain and single sphere flow propagators $\mathbf{T}_{\alpha}$ and $\mathbf{T}_{b}$ will be defined as :

$$
\begin{aligned}
& \mathbf{T}_{\alpha}\left(\mathbf{r}-\mathbf{r}^{\prime}\right)=\sum_{i, k=1}^{n} \delta\left(\mathbf{r}-\mathbf{R}_{\alpha i}\right) \mathbf{T}_{\alpha i, \alpha k} \delta\left(\mathbf{r}^{\prime}-\mathbf{R}_{\alpha k}\right) \\
& \mathbf{T}_{b}\left(\mathbf{r}-\mathbf{r}^{\prime}\right)=\iint d \Omega_{b} d \Omega_{b}^{\prime} \delta\left(\mathbf{r}-\mathbf{R}_{b}\right) \mathbf{T}_{b b^{\prime}} \delta\left(\mathbf{r}^{\prime}-\mathbf{R}_{b^{\prime}}\right)
\end{aligned}
$$

It is worth noticing that both operators still depend upon the centers of mass through $\mathbf{R}_{\alpha i}=\mathbf{R}_{\alpha}^{0}+\mathbf{S}_{\alpha i}$ and $\mathbf{R}_{b}=\mathbf{R}_{b}^{0}+\mathbf{r}_{b}\left(\Omega_{b}\right)$.

Eventually, the above definitions allow us to write (A8) in a physically more suggestive manner, as a sequence of multiple convolutions that are representing the scattering events :

$$
\begin{aligned}
& \mathbf{v}(\mathbf{r})=\mathbf{G} * \mathbf{F}+\sum_{\alpha} \mathbf{G} *\left\{\mathbf{T}_{\alpha} * \mathbf{G} * \mathbf{F}+\sum_{b} \mathbf{G} * \mathbf{T}_{b} * \mathbf{G} * \mathbf{F}\right. \\
& \quad+\sum_{\alpha} \sum_{b} \mathbf{G} *\left\{\mathbf{T}_{\alpha} * \mathbf{G} * \mathbf{T}_{b}+\mathbf{T}_{b} * \mathbf{G} * \mathbf{T}_{\alpha}\right\} * \mathbf{G} * \mathbf{F} \\
& \quad+\sum_{\alpha} \sum_{\beta(\neq \alpha)} \mathbf{G} * \mathbf{T}_{\alpha} * \mathbf{G} * \mathbf{T}_{\beta} * \mathbf{G} * \mathbf{F} \\
& \quad+\sum_{b} \sum_{c(\neq b)} \mathbf{G} * \mathbf{T}_{b} * \mathbf{G} * \mathbf{T}_{c} * \mathbf{G} * \mathbf{F}+\ldots
\end{aligned}
$$

where the dependence on $\mathbf{r}$ of the multiple convolutions is implied and the sum is continued over all permissible scattering sequences.

This is Eq. (31) we wished to derive.

\section{APPENDIX B: SELF-CONSISTENT CALCULATION OF THE W(k) TENSOR FOR $\mathbf{N}_{S}$ SPHERES IMMERSED IN A FLUID}

The main tool we need is the following expansion in spherical harmonics :

$$
\begin{aligned}
\mathcal{A}\left(\mathbf{r}(\Omega), \mathbf{r}^{\prime}\left(\Omega^{\prime}\right)\right) & =\sum_{l, l^{\prime} \geq 0}^{\infty} \sum_{m=-l}^{l} \sum_{m^{\prime}=-l^{\prime}}^{l^{\prime}} \tilde{\mathcal{A}}_{l m ; l^{\prime} m^{\prime}} Y_{l m}(\Omega) Y_{l^{\prime} m^{\prime}}^{*}\left(\Omega^{\prime}\right) \\
\tilde{\mathcal{A}}_{l m ; l^{\prime} m^{\prime}} & =\iint d \Omega d \Omega^{\prime} \mathcal{A}\left(\Omega, \Omega^{\prime}\right) Y_{l m}^{*}(\Omega) Y_{l^{\prime} m^{\prime}}\left(\Omega^{\prime}\right)(\mathrm{B} 1)
\end{aligned}
$$

where $\mathbf{r}$ and $\mathbf{r}^{\prime}$ are the position vectors for points on the surface of a sphere $\left(|\mathbf{r}|=\left|\mathbf{r}^{\prime}\right|=a\right)$ with angular directions $\Omega$ and $\Omega^{\prime}, Y_{l m}(\Omega)$ are the spherical harmonics and $\mathcal{A}$ is any tensor (vector)-like quantity.

Consider now $\mathrm{N}_{P}-1$ fixed, non-interacting spheres of radius $a$ immersed in a pure fluid of viscosity $\eta_{0}$. One can calculate the exact self-energy $\boldsymbol{\Sigma}^{S P}(\mathbf{k})$ of the suspension as in Sec. IIB (dropping the factors related to the polymer chains) but a more direct approach is to seek a self-consistent approximation, $\mathbf{W}^{S P}(\mathbf{k})$, of the exact self-energy. As discussed in Sec. IIA, we can replace our system of spheres and pure fluid averaged over the distribution of the spheres, with an effective medium in which the force propagator is $\mathcal{G}^{S P}(\mathbf{k})$ given by 44$)$. Adding one more sphere, its contribution to the approximate self-energy of the fluid is found from the second factor in (43) with the replacements $c^{\mathrm{POL}} \rightarrow 1 / V$ and $\mathbf{K}^{-1} \rightarrow \mathcal{K}^{-1}$ 


$$
\begin{aligned}
\frac{\mathbf{W}^{S P}(\mathbf{k})}{\mathrm{N}_{S}-1}=- & \frac{1}{V} \int d \Omega d \Omega^{\prime} \mathcal{K}^{-1}\left(\Omega, \Omega^{\prime}\right) \\
& \times \exp \left[i \mathbf{k} \cdot\left(\mathbf{r}(\Omega)-\mathbf{r}^{\prime}\left(\Omega^{\prime}\right)\right)\right]
\end{aligned}
$$

where $\mathcal{K}^{-1}$ is the single sphere generalized inverse of the modified Oseen tensor $\mathcal{G}$ :

$$
\int d \Omega d \Omega^{\prime \prime} \mathcal{K}^{-1}\left(\Omega, \Omega^{\prime \prime}\right) \cdot \mathcal{G}\left(\mathbf{r}\left(\Omega^{\prime \prime}\right), \mathbf{r}^{\prime}\left(\Omega^{\prime}\right)\right)=\mathbb{1} \delta\left(\Omega-\Omega^{\prime}\right)
$$

Expanding in (B2) in spherical harmonics we get :

$$
\begin{aligned}
\mathbf{W}^{S P}(\mathbf{k})= & -c^{S P} \int d \Omega d \Omega^{\prime} \sum_{l m} \sum_{l^{\prime} m^{\prime}} \mathcal{K}_{l m ; l^{\prime} m^{\prime}}^{-1} \\
& \times \exp \left[i \mathbf{k} \cdot\left(\mathbf{r}-\mathbf{r}^{\prime}\right)\right] \mathrm{Y}_{l m}(\Omega) \mathrm{Y}_{l^{\prime} m^{\prime}}^{*}\left(\Omega^{\prime}\right)
\end{aligned}
$$

But the quantities of interest (shear viscosity, diffusion coefficient) are related to the transverse part of $\mathbf{W}^{S P}(\mathbf{k})$, which should be isotropic in the $\mathbf{k}$ space (in our linearized model). Thus we can perform the angular integral in the equation above by choosing $\mathbf{k} \| \hat{\mathbf{z}}$ direction and employing the plane-wave expansion :

$$
\begin{gathered}
\exp \left[i k\left(z-z^{\prime}\right)\right]=\sum_{l, l^{\prime}=0}^{\infty}(-1)^{l^{\prime}} i^{l+l^{\prime}}(2 l+1)\left(2 l^{\prime}+1\right) \\
\times j_{l}(k a) j_{l^{\prime}}(k a) P_{l}(\cos \theta) P_{l^{\prime}}\left(\cos \theta^{\prime}\right) \\
\cos \theta=\hat{\mathbf{k}} \cdot \hat{\mathbf{r}} ; \quad \cos \theta^{\prime}=\hat{\mathbf{k}} \cdot \hat{\mathbf{r}^{\prime}} .
\end{gathered}
$$

we arrive at :

$$
\begin{aligned}
\mathbf{W}^{S P}(\mathbf{k})= & -4 \pi c^{S P} \sum_{l=0}^{\infty} \sum_{l^{\prime}=0}^{\infty}(-1)^{l^{\prime}} i^{l+l^{\prime}} \sqrt{(2 l+1)} \\
& \times \sqrt{\left(2 l^{\prime}+1\right)} j_{l}(k a) j_{l^{\prime}}(k a) \mathcal{K}_{l 0 ; l^{\prime} 0}^{-1}
\end{aligned}
$$

Note that this equation is valid for any $k$ values. A particular value of the self-energy $\mathbf{W}^{S P}(\mathbf{k})$ that is meaningful and readily obtainable from (B4), is :

$$
\mathbf{W}^{S P}(k=0)=-4 \pi c^{S P} \mathcal{K}_{00 ; 00}^{-1}
$$

For evaluating $\mathcal{K}_{l m ; l^{\prime} m^{\prime}}^{-1}$, we start by expanding the definition (B3) in spherical harmonics to transform it in the equivalent form :

$$
\sum_{l^{\prime \prime}=0}^{\infty} \sum_{m^{\prime \prime}=-l^{\prime \prime}}^{l^{\prime \prime}} \mathcal{K}_{l m ; l^{\prime \prime} m^{\prime \prime}}^{-1} \cdot \mathcal{G}_{l^{\prime \prime} m^{\prime \prime} ; l^{\prime} m^{\prime}}=\mathbb{1} \delta_{l l^{\prime}} \delta_{m m^{\prime}}
$$

where the coefficients of the Oseen tensor expansion in spherical harmonics are :

$$
\mathcal{G}_{l m ; l^{\prime} m^{\prime}}=\iint d \Omega d \Omega^{\prime} \mathcal{G}\left[\mathbf{r}(\Omega), \mathbf{r}^{\prime}\left(\Omega^{\prime}\right)\right] \mathrm{Y}_{l m}^{*}(\Omega) \mathrm{Y}_{l^{\prime} m^{\prime}}\left(\Omega^{\prime}\right)
$$

Substituting $\mathcal{G}\left(\mathbf{r}, \mathbf{r}^{\prime}\right)=\mathcal{G}\left(\mathbf{r}-\mathbf{r}^{\prime}\right)$ with its Fourier transform (calculated as in (2)) yields :

$\mathcal{G}_{l m ; l^{\prime} m^{\prime}}=\frac{2}{\pi}(-1)^{l} i^{l+l^{\prime}} \int_{0}^{\infty} d k k^{2} \frac{\mathrm{j}_{l}(k a) \mathrm{j}_{l^{\prime}}(k a)}{\eta_{0} k^{2}-W_{\perp}^{S P}(k)} \mathbb{P}_{l m ; l^{\prime} m^{\prime}}$

with $\mathrm{j}_{l}(k a)$ the spherical Bessel functions of order $l$ and $\mathbb{P}_{l m ; l^{\prime} m^{\prime}}$ the tensor :

$$
\mathbb{P}_{l m ; l^{\prime} m^{\prime}}=\int d \Omega_{k}(\mathbb{1}-\hat{\mathbf{k}} \hat{\mathbf{k}}) \mathrm{Y}_{l m}^{*}\left(\Omega_{k}\right) \mathrm{Y}_{l^{\prime} m^{\prime}}\left(\Omega_{k}\right)
$$

Now inserting $W_{\perp}^{S P}(k)$ from (45) and integrating over the $k$ variable produces 15$]$ :

$$
\begin{aligned}
\mathcal{G}_{l m ; l^{\prime} m^{\prime}}= & \frac{1}{\eta_{0} a\left(1+W_{1}^{S P}\right)} \mathrm{I}_{l+\frac{1}{2}}\left(\frac{a}{\xi^{\prime}}\right) \mathrm{K}_{l^{\prime}+\frac{1}{2}}\left(\frac{a}{\xi^{\prime}}\right) \\
& \times\left(\delta_{l l^{\prime}}+\delta_{l l^{\prime}+2}\right) \mathbb{P}_{l m ; l^{\prime} m^{\prime}}
\end{aligned}
$$

Here, I and $\mathrm{K}$ are the modified Bessel functions of the first kind and $\xi^{\prime}$ is the effective screening length in the suspension of spheres from (46).

The construction of the inverse $\mathcal{K}_{l m: l^{\prime} m^{\prime}}^{-1}$ requires an involved procedure, described in Refs. [7, 15]. It is based on the observation that $\mathcal{G}$-in its matrix representationcan be decomposed into a block diagonal $\left(l=l^{\prime}\right) \mathcal{G}_{D}$ and an off-diagonal $\mathcal{G}_{O D}$ part $\left(l \neq l^{\prime}\right)$. We could write then formally $\mathcal{G}=\mathcal{G}_{D}+\mathcal{G}_{O D}$, which leads to, by taking the inner product from left and right with $\mathcal{K}^{-1}$ and $\mathcal{K}_{D}^{-1}$,respectively, and iterating indefinitely :

$$
\mathcal{K}^{-1}=\mathcal{K}_{D}^{-1}-\mathcal{K}_{D}^{-1} \cdot \mathcal{G}_{O D} \cdot \mathcal{K}_{D}^{-1}+\ldots
$$

We compute then first $\left(\mathcal{K}_{D}^{-1}\right)_{l m ; l m^{\prime}}$ :

$$
\left(\mathcal{K}_{D}^{-1}\right)_{l m ; l m^{\prime}}=\eta_{0} a\left(1+W_{1}^{S P}\right) \frac{1}{\mathrm{I}_{l+\frac{1}{2}}\left(\frac{a}{\xi^{\prime}}\right) \mathrm{K}_{l+\frac{1}{2}}\left(\frac{a}{\xi^{\prime}}\right)} \mathbb{P}_{l m ; l m^{\prime}}^{-1}
$$

in which the last factor is the generalized inverse of $\mathbb{P}_{l m ; l m^{\prime}}$ defined by the relation :

$$
\sum_{m^{\prime \prime}=-l}^{l} \mathbb{P}_{l m ; l m^{\prime \prime}}^{-1} \cdot \mathbb{P}_{l m^{\prime \prime} ; l m^{\prime}}=\mathbb{1} \delta_{m m^{\prime}}
$$

and then one can calculate the non-diagonal part $(l=$ $\left.l^{\prime}+2\right)$ of $\mathcal{K}_{l m ; l^{\prime} m^{\prime}}^{-1}$ : 


$$
\mathcal{K}_{l m ; l^{\prime} m^{\prime}}^{-1}=\frac{\eta_{0} a\left(1+W_{1}^{S P}\right)}{\mathrm{I}_{l^{\prime}+\frac{1}{2}}\left(\frac{a}{\xi^{\prime}}\right) \mathrm{K}_{l+\frac{1}{2}}\left(\frac{a}{\xi^{\prime}}\right)} \mathbb{P}_{l m ; l^{\prime} m^{\prime}}^{-1}
$$

Finally, we return to $(\mathrm{B} 6)$ to limit the expansion to factors of order $k^{2}$ and using also the constraint $l-l^{\prime}=\{0,2\}$ we get :

$$
\begin{aligned}
\mathbf{W}^{S P}(k \ll 1) & \simeq-4 \pi c^{S P}\left[\mathcal{K}_{00 ; 00}^{-1} \mathrm{j}_{0}^{2}(k a)+3 \mathcal{K}_{10 ; 10}^{-1} \mathrm{j}_{1}^{2}(k a)\right. \\
& \left.-2 \sqrt{5} \mathrm{j}_{2}(k a) \mathrm{j}_{0}(k a) \operatorname{Re} \mathcal{K}_{20 ; 00}^{-1}\right]
\end{aligned}
$$

The actual values of the operators $\mathcal{K}_{l m ; l^{\prime} m^{\prime}}^{-1}$ are :

$$
\begin{aligned}
\mathcal{K}_{00 ; 00}^{-1} & =\frac{3}{2} a \eta_{0} \frac{1+W_{1}^{S P}}{\mathrm{I}_{1 / 2}\left(\frac{a}{\xi^{\prime}}\right) \mathrm{K}_{1 / 2}\left(\frac{a}{\xi^{\prime}}\right)} \mathbb{1} \\
\mathcal{K}_{10 ; 10}^{-1} & =\frac{1}{9} a \eta_{0} \frac{1+W_{1}}{\mathrm{I}_{3 / 2}\left(\frac{a}{\xi^{\prime}}\right) \mathrm{K}_{3 / 2}\left(\frac{a}{\xi^{\prime}}\right)} \mathbb{L}_{10 ; 10}^{-1} \\
\mathcal{K}_{20 ; 00}^{-1} & =\frac{3}{8 \sqrt{30}} a \eta_{0} \frac{1+W_{1}^{S P}}{\mathrm{I}_{1 / 2}\left(\frac{a}{\xi^{\prime}}\right) \mathrm{K}_{5 / 2}\left(\frac{a}{\xi^{\prime}}\right)} \mathbb{L}_{20 ; 00}^{-1}
\end{aligned}
$$

with the $\mathbb{L}^{-1}$ tensors given by :

$$
\begin{aligned}
& \mathbb{L}_{10 ; 00}^{-1}=12 \mathbb{1}-2 \hat{\mathbf{z}} \hat{\mathbf{z}} \\
& \mathbb{L}_{20 ; 00}^{-1}=-8 \sqrt{\frac{3}{2}} \mathbb{1}+24 \sqrt{\frac{3}{2}} \hat{\mathbf{z}} \hat{\mathbf{z}}
\end{aligned}
$$

The last step in obtaining the self-energy of the suspension of fixed spheres is to expand the spherical Bessel functions in (B17) and, collecting all factors, we get :

$$
\begin{aligned}
& \mathbf{W}^{S P}(\mathbf{k})=-6 \pi c^{S P} a \eta_{0} \frac{1+W_{1}^{S P}}{\mathrm{I}_{1 / 2}\left(\frac{a}{\xi^{\prime}}\right) \mathrm{K}_{1 / 2}\left(\frac{a}{\xi^{\prime}}\right)} \mathbb{1} \\
& +\frac{3}{2} \Phi^{S P}\left(1+W_{1}^{S P}\right) k^{2}\left[\frac{1}{\mathrm{I}_{1 / 2}\left(\frac{a}{\xi^{\prime}}\right) \mathrm{K}_{1 / 2}\left(\frac{a}{\xi^{\prime}}\right)} \mathbb{1}\right. \\
& -\frac{2}{27} \frac{1}{\mathrm{I}_{3 / 2}\left(\frac{a}{\xi^{\prime}}\right) \mathrm{K}_{3 / 2}\left(\frac{a}{\xi^{\prime}}\right)}(12 \mathbb{1}-2 \hat{\mathbf{z}} \hat{\mathbf{z}}) \\
& \left.+\frac{1}{10 \sqrt{6}} \frac{1}{\mathrm{I}_{1 / 2}\left(\frac{a}{\xi^{\prime}}\right) \mathrm{K}_{5 / 2}\left(\frac{a}{\xi^{\prime}}\right)}\left(-8 \sqrt{\frac{3}{2}} \mathbb{1}+24 \sqrt{\frac{3}{2}} \hat{\mathbf{z}} \hat{\mathbf{z}}\right)\right]
\end{aligned}
$$

that reduces immediately to (47), the equation we set to derive.

\section{APPENDIX C: SELF-CONSISTENT CALCULATION OF $\mathbf{W}^{P O L}(\mathbf{k})$ FOR A SUSPENSION OF $\mathrm{N}_{P}$ MOBILE POLYMER CHAINS}

In order to compute the self-energy of a solution of polymers, we need to make the transformations : a) change the variable $\mathbf{R}_{i}$ to $\mathbf{R}(s)$ where $s$ is the position of the $i$-th bead measured as the length of the arc along the chain, that is $i=\frac{s}{l}, l$ being the Kuhn length.

b) convert the sum $\sum_{i=1}^{n}(\cdots)$ into the integral $\frac{1}{l} \int_{0}^{l} d s(\cdots)$,and the Kronecker delta $\delta_{i j}$ to $l \delta(s-$ $s^{\prime}$.

c) the Fourier transform of a quantity $\left\langle\boldsymbol{A}\left(s, s^{\prime}\right)\right\rangle$ - the average being taken over the distribution of the segments about the center of mass of one polymer chain (assumed to be Gaussian) :

$$
\begin{aligned}
\left\langle\mathbf{A}\left(s, s^{\prime}\right)\right\rangle= & \sum_{q, q^{\prime}=-\infty}^{+\infty} \mathbf{A}_{q q^{\prime}} \exp \left(\frac{2 i \pi q s}{L}-\frac{2 i \pi q^{\prime} s^{\prime}}{L}\right) \\
\mathbf{A}_{q q^{\prime}}= & \frac{1}{L^{2}} \int_{0}^{L} d s \int_{0}^{L} d s^{\prime}\left\langle\mathbf{A}\left(s, s^{\prime}\right)\right\rangle \quad(\mathrm{C} 1) \\
& \times \exp \left(-\frac{2 i \pi q s}{L}+\frac{2 i \pi q^{\prime} s^{\prime}}{L}\right)
\end{aligned}
$$

The generalized single chain inverse $\mathcal{K}_{\alpha}^{-1}\left(\mathbf{S}_{i}, \mathbf{S}_{j}\right)$ of the modified Oseen tensor $\mathcal{G}_{\alpha}\left(\mathbf{S}_{i}, \mathbf{S}_{j}\right)$ of the polymer solution is defined by the relation :

$$
\sum_{j=1}^{n} \mathcal{K}_{\alpha}^{-1}\left(\mathbf{S}_{i}, \mathbf{S}_{j}\right) \cdot \mathcal{G}\left(\mathbf{S}_{\alpha j}, \mathbf{S}_{\alpha k}\right)=\delta_{i k} \mathbb{1}
$$

Averaging over segments distribution and applying the previous transformations we get :

$$
\frac{1}{l^{2}} \int_{0}^{L} d s^{\prime} \mathcal{K}_{\alpha}^{-1}\left(s, s^{\prime}\right) \cdot \mathcal{G}\left(s^{\prime}, s^{\prime \prime}\right)=\delta\left(s-s^{\prime \prime}\right) \mathbb{1}
$$

the Fourier transform of which being :

$$
\sum_{q^{\prime}=-\infty}^{+\infty} \mathcal{K}_{q q^{\prime}}^{-1} \cdot \mathcal{G}_{q^{\prime} q^{\prime \prime}}=\frac{1}{n^{2}} \delta_{q q^{\prime \prime}} \mathbb{1}
$$

or, in operator notation $\mathcal{K}^{-1}=\frac{1}{n^{2}} \mathcal{G}^{-1}$.

Our starting point is Eq. (54) converted to an integral form :

$$
\begin{gathered}
\mathbf{W}^{P O L}(\mathbf{k})=-c^{P O L} \frac{1}{l^{2}} \int_{0}^{L} d s \int_{0}^{L} d s^{\prime}\langle\exp [i \mathbf{k} \cdot(\mathbf{R}(s) \\
\left.\left.\left.-\mathbf{R}\left(s^{\prime}\right)\right)\right]\right\rangle\left\{\left\langle\mathcal{K}^{-1}\left(s, s^{\prime}\right)\right\rangle\right. \\
\left.-\frac{1}{l^{2}}\left\langle\mathbf{g}_{t}^{-1}\right\rangle \cdot \int_{0}^{L} \int_{0}^{L} d p d p^{\prime}\left\langle\mathcal{K}^{-1}(s, p)\right\rangle \cdot\left\langle\mathcal{K}^{-1}\left(p^{\prime}, s^{\prime}\right)\right\rangle\right\}
\end{gathered}
$$

where the averages are taken over the distribution of the segments about the center of mass of the polymer chain. 
For a Gaussian probability distribution function we have the following result [10]:

$$
\left\langle\exp \left[i \mathbf{k} \cdot\left(\mathbf{R}(s)-\mathbf{R}\left(s^{\prime}\right)\right)\right]\right\rangle=\exp \left[-\frac{k^{2} l\left|s-s^{\prime}\right|}{6}\right]
$$

Inserting the above expression in (C5), Fourier expanding the $\left\langle\mathcal{K}^{-1}\right\rangle$ factors and using their definition gives :

$$
\begin{aligned}
& \mathbf{W}^{P O L}(\mathbf{k})=\mathbf{W}_{a}^{P O L}(\mathbf{k})+\mathbf{W}_{b}^{P O L}(\mathbf{k}) \\
& \mathbf{W}_{a}^{P O L}(\mathbf{k})=-c^{P O L} \sum_{q, q^{\prime}=-\infty}^{+\infty} \mathrm{I}_{q q^{\prime}}^{*}(k) \mathcal{G}_{q q^{\prime}}^{-1} \mathbb{1} \\
& \mathbf{W}_{b}^{P O L}(\mathbf{k})=c^{P O L}\left\langle g_{t}^{-1}\right\rangle \frac{n^{2}}{l^{2}} \int_{0}^{L} \int_{0}^{L} d p d p^{\prime} \sum_{q, q^{\prime}}^{+\infty} \sum_{\omega, \omega^{\prime}}^{+\infty} \\
& \mathrm{I}_{q q^{\prime}}(k) \exp \left(-\frac{2 i \pi \omega p}{L}+\frac{2 i \pi \omega^{\prime} p^{\prime}}{L}\right) \mathcal{K}_{q \omega}^{-1} \mathcal{K}_{\omega^{\prime} q^{\prime}}^{-1} \mathbb{1}
\end{aligned}
$$

with the notation :

$$
\begin{aligned}
\mathrm{I}_{q q^{\prime}}^{*}(k)= & \frac{1}{L^{2}} \int_{0}^{L} \int_{0}^{L} d s d s^{\prime} \exp \left[-\frac{k^{2} l\left|s-s^{\prime}\right|}{6}\right] \\
& \times \exp \left[\frac{2 i \pi q s}{L}-\frac{2 i \pi q^{\prime} s^{\prime}}{L}\right]
\end{aligned}
$$

In the previous relations we implicitly assumed that all tensors are multiples of the unit tensor, which will be shown to be true under certain approximations.

The integrals over $p, p^{\prime}$ in the $\mathbf{W}_{b}^{P O L}$ expression are straightforward, yielding :

$$
\mathbf{W}_{b}^{P O L}(\mathbf{k})=c^{P O L}\left\langle g_{t}^{-1}\right\rangle \sum_{q q^{\prime}} \mathrm{I}_{q q^{\prime}}^{*}(k) \mathcal{G}_{q 0}^{-1} \mathcal{G}_{0 q^{\prime}}^{-1} \mathbb{1}
$$

Now $\mathrm{I}_{q q^{\prime}}^{*}(k)$ can be evaluated to be :

$$
\begin{aligned}
& \mathrm{I}_{q q^{\prime}}^{*}(k)=\frac{2}{L^{2}}\left\{\left(\frac{L}{2 \pi q}\right)^{2} \frac{k^{2} R_{g}^{2}}{1+\left(\frac{k^{2} R_{g}^{2}}{2 \pi q}\right)^{2}} \delta_{q q^{\prime}}\right. \\
& -\frac{\left[\left(\frac{k^{2} R_{g}^{2}}{2 \pi}\right)^{2}-q q^{\prime}\right]}{\left(\frac{2 \pi}{L}\right)^{2} q^{2} q^{\prime 2}\left[1+\left(\frac{k^{2} R_{g}^{2}}{2 \pi q}\right)^{2}\right]\left[1+\left(\frac{k^{2} R_{g}^{2}}{2 \pi q^{\prime}}\right)^{2}\right]} \\
& \left.\times\left[1-\exp \left(-k^{2} R_{g}^{2}\right)\right]\right\}
\end{aligned}
$$

in which $R_{g}=\sqrt{\frac{n l^{2}}{6}}$ is the radius of gyration of any polymer chain. What remains to be calculated is the $\mathcal{G}^{-1}$ operator.

Approximating the transverse component of $\mathbf{W}^{P O L}(\mathbf{k})$ with (53) and using the translational invariance of the suspension we compute first the effective Oseen tensor :

$$
\begin{aligned}
\mathcal{G}\left(\mathbf{R}(s), \mathbf{R}\left(s^{\prime}\right)\right)= & \mathcal{G}\left(\mathbf{R}_{s}-\mathbf{R}_{s}^{\prime}\right) \\
= & \int_{\mathbf{k}} \mathcal{G}(\mathbf{k}) \exp \left[-i \mathbf{k} \cdot\left(\mathbf{R}_{s}-\mathbf{R}_{s}^{\prime}\right)\right] \\
\mathcal{G}(\mathbf{k})= & \frac{\mathbb{1}-\hat{\mathbf{k}} \hat{\mathbf{k}}}{\eta_{0} k^{2}-W_{\perp}^{P O L}(\mathbf{k})} \\
= & \frac{\hat{\mathbb{1}}-\hat{\mathbf{k}} \hat{\mathbf{k}}}{\eta_{0}\left(1+W_{1}^{P O L}\right)\left(k^{2}+\xi_{P O L}^{\prime-2}\right)} \\
& \xi_{P O L}^{\prime-2}=\frac{\xi^{-2}}{1+W_{1}^{P O L}}
\end{aligned}
$$

where the Fourier integral is given by (2) and $\xi_{P O L}^{\prime}$ is the effective screening length in the polymer solution.

Averaging over the distribution of the segments and integrating over the solid angle, recalling that $W_{\perp}^{P O L}(k)$ does not depend on the direction of $\mathbf{k}$, we get :

$$
\begin{aligned}
\left\langle\mathcal{G}\left(s, s^{\prime}\right)\right\rangle= & \frac{1}{3 \pi^{2}} \int_{0}^{\infty} d k k^{2} \frac{1}{\eta_{0} k^{2}-W_{\perp}^{P O L}(k)} \\
& \times \exp \left(-\frac{k^{2} l\left|s-s^{\prime}\right|}{6}\right) \mathbb{1}
\end{aligned}
$$

Then, applying the Fourier transform defined at the beginning of the appendix, one finds :

$$
\mathcal{G}_{q q^{\prime}}=\frac{1}{3 \pi^{2}} \int_{0}^{\infty} d k \frac{k^{2}}{\eta_{0}\left(1+W_{1}^{P O L}\right)\left(k^{2}+\xi_{P O L}^{\prime-2}\right)} \mathrm{I}_{q q^{\prime}}(k) \mathbb{1}
$$

where we used $\int d \Omega_{k}(\mathbb{1}-\hat{\mathbf{k}} \hat{\mathbf{k}})=\frac{8 \pi}{3} \mathbb{1}$ and $\mathrm{I}_{q q^{\prime}}$ has been evaluated in ( $\mathrm{C} 10$ ).

In order to make the calculation of $\mathcal{G}$ analytically tractable, without coarsening the physical picture too much, we will make two approximations :

1. the Kirkwood-Riseman approximation,which amounts to discard the off-diagonal terms in $\mathrm{I}_{q q^{\prime}}^{*}(k)$.

2. the long chain limit, $n \gg 1$.

Under these assumptions, both $\mathcal{G}$ and its inverse are diagonal and their Fourier elements are simply related as :

$$
\left(\mathcal{G}^{-1}\right)_{q q^{\prime}}=\left(\mathcal{G}^{-1}\right)_{q} \delta_{q q^{\prime}}=\left(\mathcal{G}_{q}\right)^{-1} \delta_{q q^{\prime}}
$$

For convenience we will denote these matrix elements by $\mathcal{G}_{q}^{-1}$.

We will proceed further to evaluate $\mathbf{W}^{P O L}(\mathbf{k})$. Noticing that $\mathrm{I}_{q}=\mathrm{I}_{-q}$, one finds :

$$
\begin{aligned}
& \mathbf{W}_{a}^{P O L}(k)=-c^{P O L}\left\{\mathrm{I}_{0}(k) \mathcal{G}_{0}^{-1}+2 \sum_{q=1}^{\infty} \mathrm{I}_{q}(k) \mathcal{G}_{q}^{-1}\right\} \mathbb{1} \\
& \mathbf{W}_{b}^{P O L}(k)=c^{P O L} \mathrm{I}_{0}(k) \mathcal{G}_{0}^{-1} \mathbb{1}
\end{aligned}
$$


in which we obtained $\left\langle\mathbf{g}_{t}^{-1}\right\rangle$ from :

$$
\begin{aligned}
\left\langle\mathbf{g}_{t}\right\rangle & =\sum_{i, j=1}^{n}\left\langle\mathcal{K}_{i j}^{-1}\right\rangle=\frac{1}{L^{2}} \int_{0}^{L} \int_{0}^{L} d s d s^{\prime} \mathcal{G}^{-1}\left(s, s^{\prime}\right)=\mathcal{G}_{0}^{-1} \\
\left\langle\mathbf{g}_{t}^{-1}\right\rangle & =\mathcal{G}_{0}
\end{aligned}
$$

Working in the hydrodynamic limit $k \rightarrow 0$, we expand $\mathrm{I}_{q}(k \ll 1) \simeq \frac{1}{\pi^{2} q^{2}} k^{2} R_{g}^{2}$ and converting the sum over $q$ to an integral over the wavevector $\mu=\frac{2 \pi q}{L}$, we obtain the following expression for $\mathbf{W}^{P O L}(k)$ by adding the two terms (C15a,C15b) :

$$
\mathbf{W}^{P O L}(k)=-\frac{4}{\pi L} c^{P O L} R_{g}^{2} k^{2} \int_{\frac{2 \pi}{L}}^{\infty} d \mu \frac{1}{\mu^{2}} \mathcal{G}_{\mu}^{-1} \mathbb{1}
$$

To calculate the translational friction coefficient (and implicitly,the diffusion coefficient), we have to consider one polymer chain moving with some uniform velocity $\mathbf{u}_{0}$ inside an effective medium replacing all others $\mathrm{N}_{P}-1$ chains, medium characterized by the force propagator $\mathcal{G}(\mathbf{k})$ given by $(\mathrm{C} 11)$. The equation of motion of the center of mass of the chain is :

$$
M \ddot{\mathbf{R}}^{0}=-\sum_{i=1}^{n} \boldsymbol{\sigma}_{i}+\mathbf{f}_{0}
$$

$M$ is the mass of the chain and $\boldsymbol{\sigma}_{i}$ is the force exerted by the $i$-th bead of the polymer upon the fluid. Neglecting the inertial term (stationary problem) in the above equation of motion, the total external average force upon the polymer chain is found to be :

$$
\left\langle\mathbf{f}_{0}\right\rangle=\left\langle\sum_{i=1}^{n} \boldsymbol{\sigma}_{i}\right\rangle=\sum_{i, k}^{n}\left\langle\mathcal{K}_{i k}^{-1}\right\rangle \cdot \mathbf{u}_{0}
$$

where the expression of $\sigma_{i}$ is a particular case of (A4) no spheres present and the other chains are absorbed in the effective fluid by replacing $\mathbf{K}_{i j}^{-1}$ with $\mathcal{K}_{i j}^{-1}$. The translational friction coefficient follows readily :

$$
\zeta_{t} \mathbb{1}=\sum_{i, k}^{n}\left\langle\mathcal{K}_{i k}^{-1}\right\rangle=\sum_{i, k}^{n}\left\langle\mathcal{K}_{i k}^{-1}\right\rangle \mathbb{1}
$$

where first we had a configurational average as defined in (32) that reduced to an average over the distribution of the segments $\mathbf{S}_{i}, \mathbf{S}_{k}$ about the center of mass of the chain. Introducing the arc-length variables $s$ and $s^{\prime}$ and applying the Fourier transform we obtain for $\zeta_{t}$ :

$$
\zeta_{t}=\mathcal{G}_{0}^{-1} \mathbb{1}=\left\langle g_{t}\right\rangle \mathbb{1}
$$

Next we insert (C10) in $($ C13 $)$ and setting $q=q^{\prime}=0$ we calculate $\mathcal{G}_{0}$ :

$$
\begin{aligned}
\mathcal{G}_{0}= & \frac{2}{3 \pi^{2}} \frac{1}{\eta_{0}\left(1+W_{1}^{P O L}\right)} \frac{1}{R_{g} \beta} \int_{0}^{\infty} d x \frac{1}{x^{2}+1} \\
& \times\left\{1-\frac{1}{(\beta x)^{2}}\left[1-\exp \left(-\beta^{2} x^{2}\right)\right]\right\} \mathbb{1}
\end{aligned}
$$

with $\beta$ a dimensionless variable : $\beta=R_{g} \xi_{P O L}^{\prime-1}$. The integral gives the exact result :

$$
\begin{aligned}
& \mathcal{G}_{0}=\frac{1}{3 \pi} \frac{1}{\eta_{0}\left(1+W_{1}^{P O L}\right)} \frac{1}{R_{g}} \frac{\mathrm{P}(\beta)}{\beta} \mathbb{1} \\
& \mathrm{P}(\beta)=1+\frac{1}{\beta^{2}}\left[1-\exp \left(\beta^{2}\right)+\frac{2}{\sqrt{\pi}} \exp \left(\beta^{2}\right) \Gamma\left(\frac{3}{2}, 0, \beta^{2}\right)\right] \\
& \Gamma\left(\frac{3}{2}, 0, x\right)=\int_{0}^{x} d t t^{\frac{1}{2}} \exp (-t)
\end{aligned}
$$

where the last expression is the incomplete Gamma function.

Thus the friction coefficient is given by :

$$
\zeta_{t} \mathbb{1}=\mathcal{G}_{0}^{-1}=3 \pi \eta_{0}\left[1+W_{1}^{P O L}\left(\Phi^{P O L}\right)\right] R_{g} \frac{\beta}{\mathrm{P}(\beta)} \mathbb{1}
$$

From $(\overline{\mathrm{C} 13})$, in the long-chain limit, $\mathcal{G}_{\mu}$ becomes :

$$
\begin{aligned}
\mathcal{G}_{\mu}= & \frac{1}{3 \pi^{2}} \frac{1}{\eta_{o}\left(1+W_{1}^{P O L}\right)} \frac{2}{L^{2}} \int_{0}^{\infty} d p \frac{p^{2}}{p^{2}+\xi^{\prime-2}} \\
& \times \frac{1}{\mu^{2}} \frac{p^{2} R_{g}^{2}}{1+\left(\frac{p^{2} R_{g}^{2}}{\mu L}\right)^{2}} \mathbb{1}
\end{aligned}
$$

where $\mu=\frac{2 \pi q}{L}$. Working out the integral in terms of dimensionless variables we get :

$$
\mathcal{G}_{\mu}=\frac{1}{6 \pi} \frac{1}{\eta_{0}\left(1+W_{1}^{P O L}\right)} \frac{\sqrt{2}}{R_{g} \beta} \frac{\sqrt{2}-\sqrt{\frac{\mu L}{\beta^{2}}}+\left(\frac{\mu L}{\beta^{2}}\right)^{3 / 2}}{1+\left(\frac{\mu L}{\beta^{2}}\right)^{2}} \mathbb{1}
$$

Also, $\mathcal{G}_{\mu}^{-1}$ is immediately computed as : $\mathcal{G}_{\mu}^{-1}=\left(\mathcal{G}_{\mu}\right)^{-1}$.

Finally, changing the integration variable in (C17) from $\mu$ to $x=\left(\mu L / \beta^{2}\right)$ and defining the volume fraction of the polymers to be $\Phi^{P O L}=\frac{4 \pi R_{g}^{3}}{3} c^{P O L}$, we calculate $\mathbf{W}^{P O L}(\mathbf{k})$ :

$$
\begin{aligned}
\mathbf{W}^{P O L}(\mathbf{k})= & -\frac{36}{\pi} \eta_{0}\left(1+W_{1}^{P O L}\right) \Phi^{P O L} k^{2} \frac{1}{\beta} \\
& \times \int_{\sqrt{\frac{2 \pi}{\beta^{2}}}}^{\infty} d x \frac{x^{4}+1}{\sqrt{2} x^{3}\left(x^{3}-x+\sqrt{2}\right)} \mathbb{1}
\end{aligned}
$$


where the integration can be done exactly, leading eventually to the desired equation for the self-energy $\mathbf{W}^{P O L}$ of a suspension of $\mathrm{N}_{P}$ non-interacting, mobile polymer chains :

$$
\begin{array}{r}
\mathbf{W}^{P O L}(\mathbf{k})=-\frac{9}{\pi} \eta_{0}\left(1+W_{1}^{P O L}\right) \Phi^{P O L} k^{2} \mathrm{Q}(\beta) \mathbb{1} \\
\mathrm{Q}(\beta)=\frac{1}{\beta} \ln \left(1+\frac{\beta}{\sqrt{\pi}}\right)+\frac{1}{\sqrt{\pi}}+\frac{\beta}{2 \pi}
\end{array}
$$

with $\beta=\frac{R_{g}}{\xi^{\prime} P O L}$.

As a last note, we present here the series expansions and the asymptotic behavior of the functions $\mathrm{P}(x)$ and $\mathrm{Q}(x)$ introduced in the previous calculations:

$$
\begin{gathered}
x \ll 1\left\{\begin{array}{l}
\mathrm{P}(x) \simeq \frac{4}{3 \sqrt{\pi}} x-\frac{1}{2} x^{2}+\frac{8}{15 \sqrt{\pi}} x^{3}-\ldots \\
\mathrm{Q}(x) \simeq \frac{2}{\sqrt{\pi}}+\frac{1}{3 \pi \sqrt{\pi}} x^{2}-\frac{1}{4 \pi^{2}} x^{3}+\ldots
\end{array}\right. \\
x \gg 1 \quad\left\{\begin{array}{l}
\mathrm{P}(x) \simeq 1-\frac{2}{\sqrt{\pi} x}+\frac{1}{x^{2}}-\frac{1}{\sqrt{\pi} x^{3}}+\ldots \\
\mathrm{Q}(x) \simeq \frac{1}{2 \pi} x+\frac{1}{\sqrt{\pi}}+\ldots
\end{array}\right.
\end{gathered}
$$

[1] A. Einstein, Ann. Phys. 19, 289 (1906); 34, 591 (1911); The Theory of Brownian Movement (Dover, New York, 1956).
[2] J. M. Peterson and M. J. Fixman, Chem. Phys. 39, 2516 (1963).

[3] G. K. Batchelor and J. T. Green, J. Fluid Mech. 56, 401 (1972).

[4] K. F. Freed and M. Muthukumar, J. Chem. Phys. 69 (6), 2657 (1978).

[5] K. F. Freed and M. Muthukumar, J. Chem. Phys. 76 (12), 6186 (1982).

[6] M. Muthukumar and K. F. Freed, J. Chem. Phys. 76 (12), 6195 (1982).

[7] M. Muthukumar and K. F. Freed, J. Chem. Phys. 70 (12), 5875 (1979).

[8] C. W. J. Beenaker, Physica A 128, 48 (1984).

[9] C. U. Thomas and M. Muthukumar, J. Chem. Phys. 94 (7), 5180 (1991).

[10] H. Yamakawa, Modern Theory of Polymer Solutions (Harper and Row, New York, 1971).

[11] K. F. Freed and S. F. Edwards, J. Chem. Phys. 61, 3626 (1974); 62, 4032 (1975).

[12] M. Muthukumar, J. Phys. A 14, 2129 (1981).

[13] M. Doi and S. F. Edwards, The Theory of Polymer Dynamics (Clarendon, Oxford, 1986).

[14] J. G. Kirkwood and J. Riseman, J. Chem. Phys. 16, 565 (1948).

[15] M. Muthukumar, J. Chem. Phys. 77 (2), 959 (1982).

[16] L. D. Landau and E. M. Lifshitz, Fluid Mechanics (Pergamon, New York, 1959).

[17] D. Bedeaux and P. Mazur, Physica (Utrecht) 76, 235 (1974)

[18] K. F. Freed and M. Muthukumar, J. Chem. Phys. 68 (5), 2088 (1978).

[19] L. Marshall and C. F. Zukoski, Mat. Res. Soc. Symp. Proc. 155, 65 (1989). 
FIG. 1. The physical interpretation of the multiple scattering expression of the microscopic velocity field $\mathbf{v}(\mathbf{r})$. O = observation point; $\mathbf{G}=$ the Oseen tensor of the pure fluid; $\mathbf{T}^{\mathrm{OBJ}}=$ single-object velocity propagator.

FIG. 2. Diffusion coefficient $D$ function of $\Phi^{P O L}$ for the $\mathbf{1 S} / \mathbf{P}$ limit case and for the $\mathbf{S} / \mathbf{P}$ system with $\Phi^{S P}$ parameter; all $t$ values. From top to bottom : +1 sphere limit; $\diamond \Phi^{S P}=0.01 ; \triangle \Phi^{S P}=0.11 ; \bigcirc \Phi^{S P}=0.21 ; \square \Phi^{S P}=0.31$.

FIG. 3. Diffusion coefficient $D$ function of $\Phi^{S P}$ for a probe sphere moving among other fixed spheres in a suspension of polymers ( $\mathbf{S} / \mathbf{P}$ system), with $\Phi^{P O L}$ parameter; all $t$ values. From top to bottom : $\diamond \Phi^{P O L}=0.01 ; \triangle \Phi^{P O L}=0.09$; $\bigcirc \Phi^{P O L}=0.16 ; \square \Phi^{P O L}=0.24$.

FIG. 4. Total viscosity change $\frac{\eta^{\text {eff }}}{\eta_{0}}-1$ function of $\Phi^{P O L}$ for the $\mathbf{1 S} / \mathbf{P}$ limit case and for the $\mathbf{S} / \mathbf{P}$ system with $\Phi^{S P}$ parameter; all $t$ values. From bottom to top : +1 sphere limit; $\diamond \Phi^{S P}=0.01 ; \triangle \Phi^{S P}=0.14 ; \bigcirc \Phi^{S P}=0.26 ; \square \Phi^{S P}=0.39$.

FIG. 5. Total viscosity change $\frac{\eta^{\text {eff }}}{\eta_{0}}-1$ function of $\Phi^{S P}$ for the $\mathbf{S} / \mathbf{P}$ system, with $\Phi^{P O L}$ parameter; all $t$ values. From bottom to top : $\diamond \Phi^{P O L}=0.01 ; \triangle \Phi^{P O L}=0.09$; $\bigcirc \Phi^{P O L}=0.16 ; \square \Phi^{P O L}=0.24$.

FIG. 6. Relative viscosity change $\frac{\eta^{\text {eff }}}{\eta_{\mathrm{POL}}}-1$ function of $\Phi^{P O L}$ for the $\mathbf{S} / \mathbf{P}$ system, with $\Phi^{S P}$ parameter; all $t$ values. From bottom to top : $\diamond \Phi^{S P}=0.09 ; \triangle \Phi^{S P}=0.24$; $\bigcirc \Phi^{S P}=0.39 ; \square \Phi^{S P}=0.46$.

FIG. 7. Diffusion coefficient $D$ function of $\Phi^{S P}$ for the $\mathbf{1 P} / \mathbf{S}$ limit and for the $\mathbf{P} / \mathbf{S}$ system with $\Phi^{P O L}$ parameter; $t=0.01$. From top curve to bottom curve, Figs. [7-10] : +1 chain limit; $\diamond \Phi^{P O L}=0.01 ; \triangle \Phi^{P O L}=0.09$; $\bigcirc \Phi^{P O L}=0.16 ; \square \Phi^{P O L}=0.24$.

FIG. 8. Diffusion coefficient $D$ function of $\Phi^{S P}$. $1 \mathbf{P} / \mathbf{S}$ and $\mathbf{P} / \mathbf{S}$ systems, with $\Phi^{P O L}$ parameter; $t=0.1$.

FIG. 9. Diffusion coefficient $D$ function of $\Phi^{S P}$. $1 \mathbf{P} / \mathbf{S}$ and $\mathbf{P} / \mathbf{S}$ systems, with $\Phi^{P O L}$ parameter; $t=1$.

FIG. 10. Diffusion coefficient $D$ function of $\Phi^{S P}$. $\quad \mathbf{P} / \mathbf{S}$ and $\mathbf{P} / \mathbf{S}$ systems, with $\Phi^{P O L}$ parameter; $t=10$.
FIG. 11. Differences in the diffusion coefficient of the polymer chain function of $\Phi^{S P}$ for four pairs of $t$ values $(\mathbf{1 P} / \mathbf{S}$ system). From top to bottom,following the maximum of each curve : $\bigcirc D(t=0.01)-D(t=10) ; \diamond D(t=1)-D(t=10)$; $\triangle D(t=0.01)-D(t=1) ; \square D(t=0.01)-D(t=0.1)$.

FIG. 12. Diffusion coefficient $D$ function of $\Phi^{P O L}$ for one mobile polymer chain inside a dispersion of other polymer chains and fixed spheres (P/S system), with $\Phi^{S P}$ parameter; $t=0.1$. From top to bottom , Figs. [12-14] : $\diamond \Phi^{S P}=0.01$; $\triangle \Phi^{S P}=0.14 ; \bigcirc \Phi^{S P}=0.26 ; \square \Phi^{S P}=0.39$.

FIG. 13. Diffusion coefficient $D$ function of $\Phi^{P O L}$. $\mathbf{P} / \mathbf{S}$ system, with $\Phi^{S P}$ parameter; $t=1$.

FIG. 14. Diffusion coefficient $D$ function of $\Phi^{P O L}$. $\mathbf{P} / \mathbf{S}$ system, with $\Phi^{S P}$ parameter; $t=10$.

FIG. 15. Total viscosity change $\frac{\eta^{\text {eff }}}{\eta_{0}}-1$ function of $\Phi^{P O L}$. $\mathbf{P} / \mathbf{S}$ system, with $\Phi^{S P}$ parameter; $t=0.01$. From bottom to top curve : $\diamond \Phi^{S P}=0.01 ; \triangle \Phi^{S P}=0.14 ; \bigcirc \Phi^{S P}=0.26$; $\square \Phi^{S P}=0.39$.

FIG. 16. Total viscosity change $\frac{\eta^{\text {eff }}}{\eta_{0}}-1$ function of $\Phi^{P O L}$. $\mathbf{P} / \mathbf{S}$ system, with $\Phi^{S P}$ parameter; $t=10$. From bottom to top curve : $\diamond \Phi^{S P}=0.01 ; \triangle \Phi^{S P}=0.14 ; \bigcirc \Phi^{S P}=0.26$; $\square \Phi^{S P}=0.39$.

FIG. 17. Total viscosity change $\frac{\eta^{\text {eff }}}{\eta_{0}}-1$ function of $\Phi^{S P}$. $\mathbf{P} / \mathbf{S}$ system, with $\Phi^{P O L}$ parameter; $t=0.01$. From bottom to top curve, Figs. [17-18] : $\diamond \Phi^{P O L}=0.01 ; \triangle \Phi^{P O L}=0.09$; $\bigcirc \Phi^{P O L}=0.16 ; \square \Phi^{P O L}=0.24$.

FIG. 18. Total viscosity change $\frac{\eta^{\text {eff }}}{\eta_{0}}-1$ function of $\Phi^{S P}$. $\mathbf{P} / \mathbf{S}$ system, with $\Phi^{P O L}$ parameter; $t=10$.

FIG. 19. The relative viscosity change $\frac{\eta^{\text {eff }}}{\eta_{\mathrm{SP}}}-1$ as a function of $\Phi^{P O L}$ for the $\mathbf{P} / \mathbf{S}$ system, with $\Phi^{S P}$ parameter; $t=1$ when symbols are joined with lines and $t=10$ when they are isolated. The corresponding $\Phi^{S P}$ values are : $\diamond$ and $\diamond-\diamond \Phi^{S P}=0.01 ; \triangle \Phi^{S P}=0.14 ; \bigcirc \Phi^{S P}=0.26 ; \square$ and $\square-\square \Phi^{S P}=0.39$.

FIG. 20. The relative viscosity change $\frac{\eta^{\text {eff }}}{\eta_{\mathrm{SP}}}-1$ as a function of $\Phi^{S P}$. P $/ \mathbf{S}$ system, with $\Phi^{P O L}$ parameter; $t=0.01$. From bottom to top curve, $\triangle \Phi^{P O L}=0.09 ; \bigcirc \Phi^{P O L}=0.16$; $\square \Phi^{P O L}=0.24$ 
FIG. 21. The relative viscosity change $\frac{\eta^{\text {eff }}}{\eta_{\mathrm{SP}}}-1$ as a function of $\Phi^{S P} . \mathbf{P} / \mathbf{S}$ system, with $\Phi^{P O L}$ parameter; $t=1$ when symbols are joined with lines and $t=10$ when they are isolated. The corresponding $\Phi^{P O L}$ values are : $\triangle$ and $\triangle-\triangle \Phi^{P O L}=0.09 ; \bigcirc$ and $\bigcirc-\bigcirc \Phi^{P O L}=0.16 ; \square$ and $\square-\square \Phi^{S P}=0.24$.

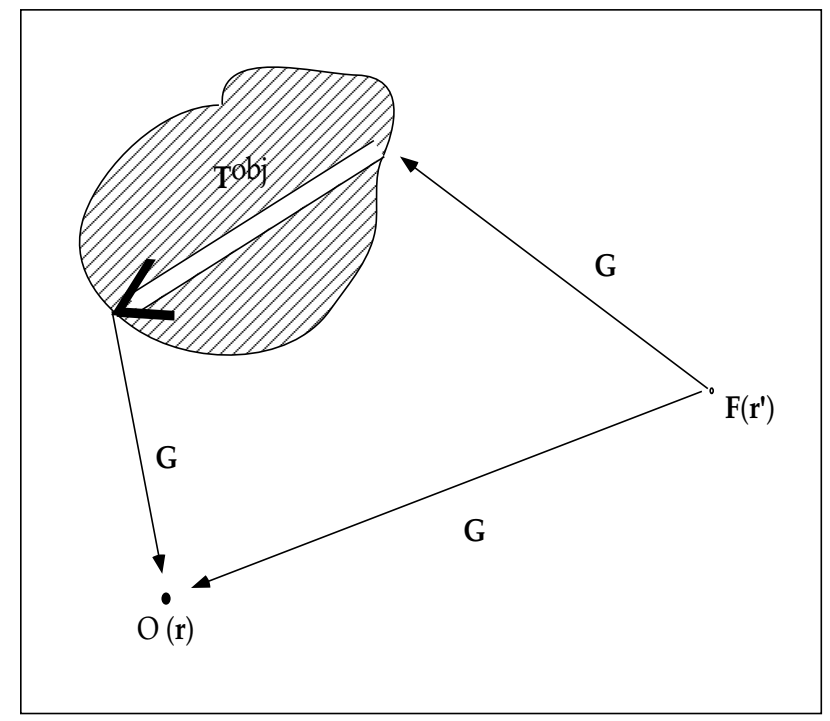

FIG. 1 
Fig. 2

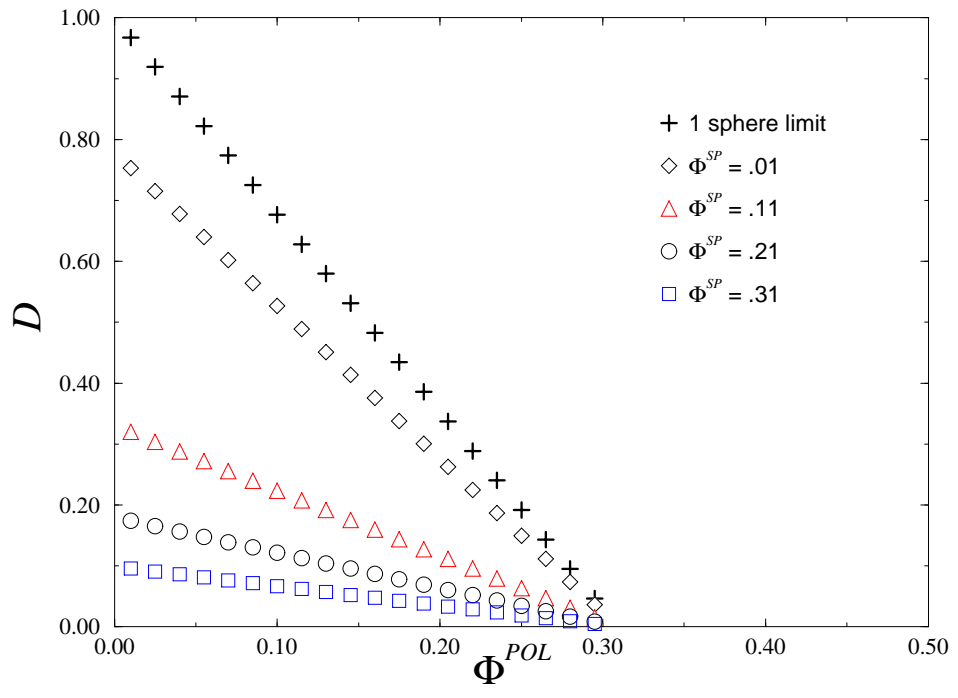

Fig. 4

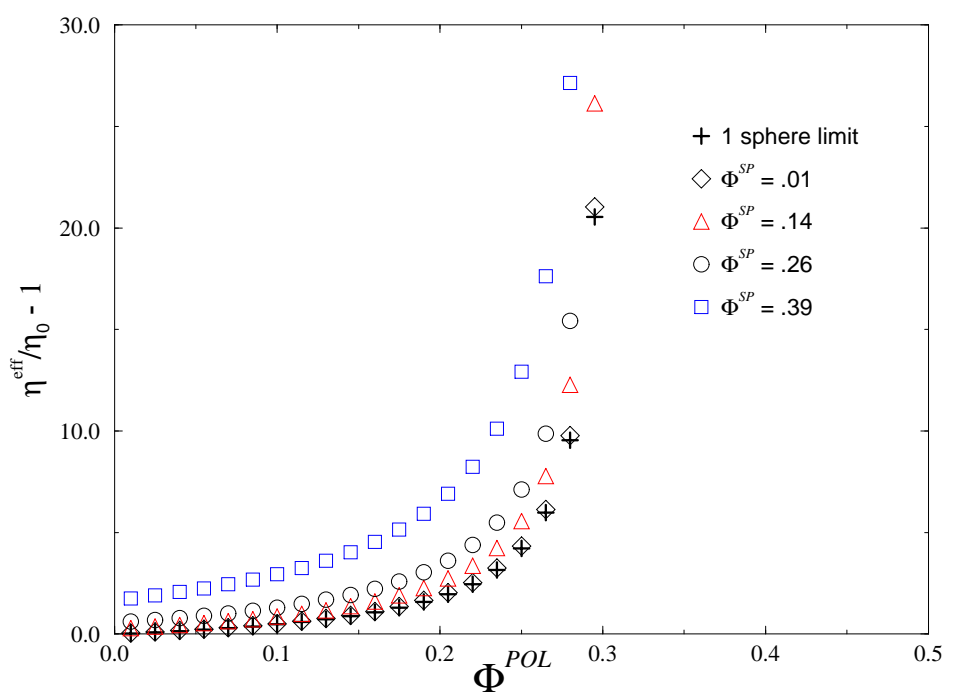

Fig. 3

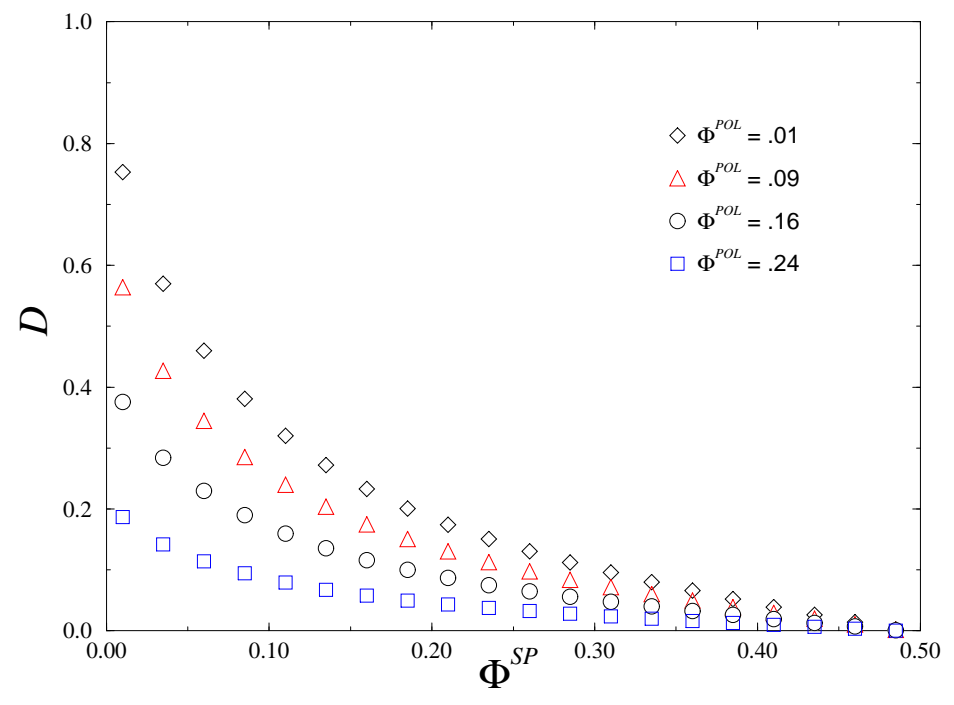

Fig. 5

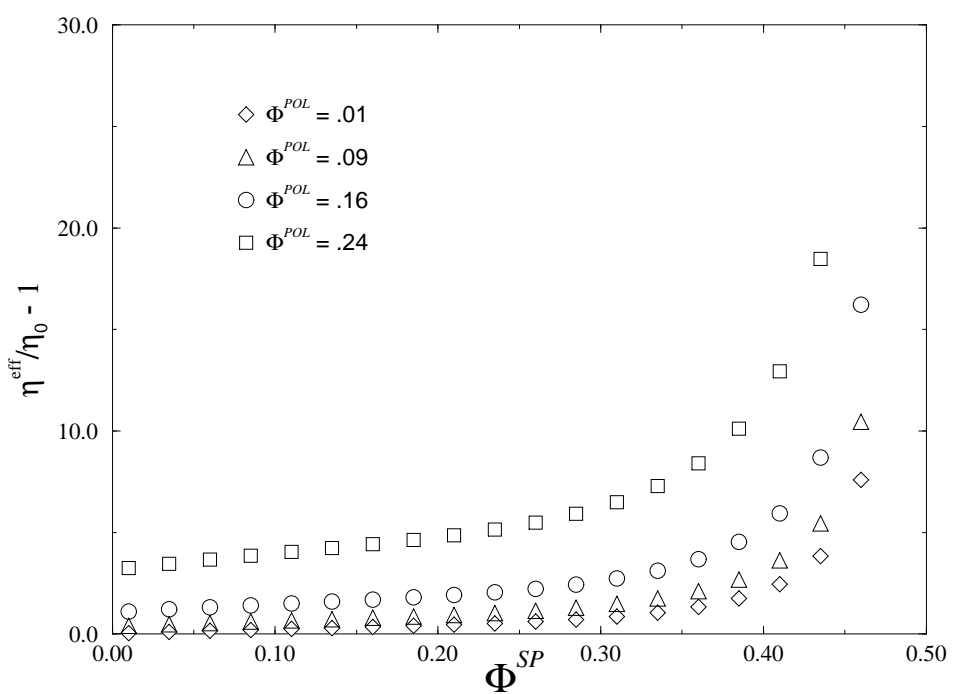


Fig. 6

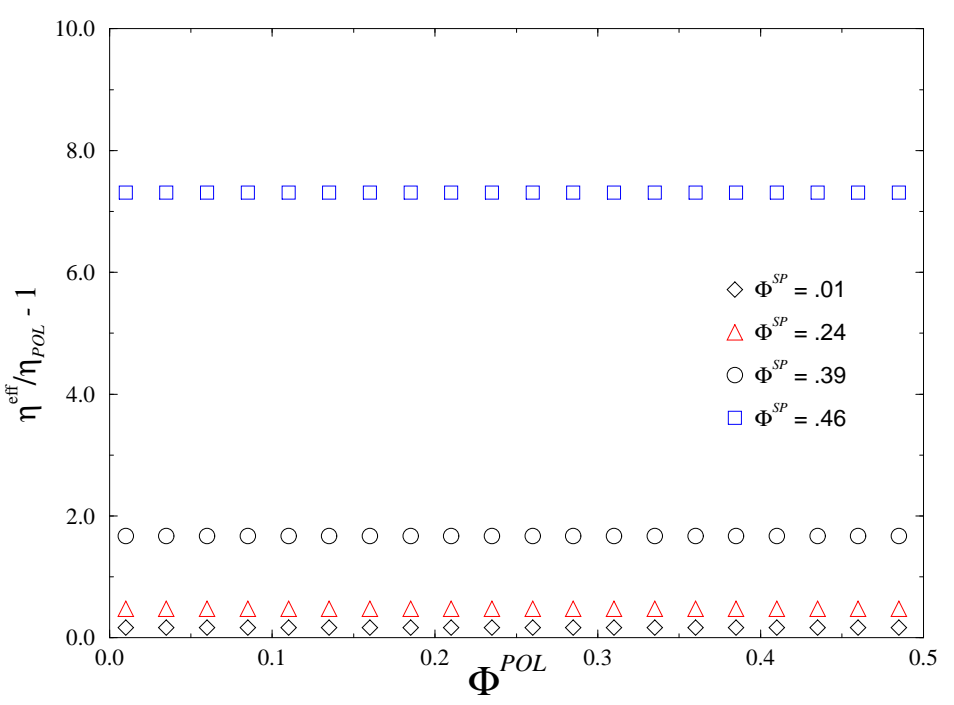

v

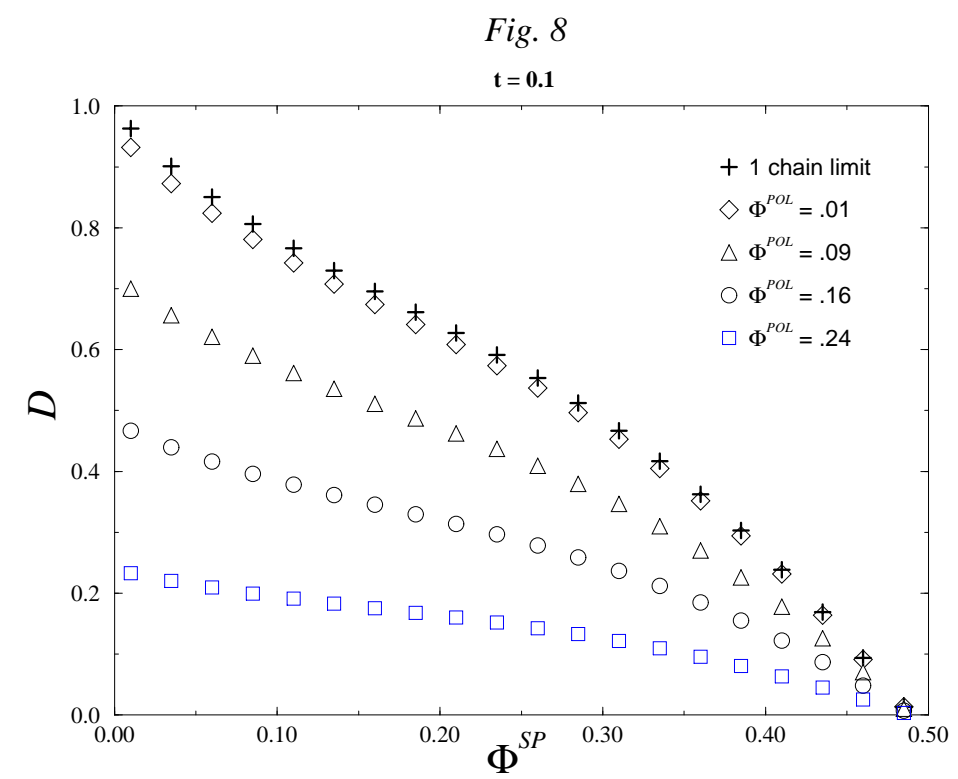

Fig. 7

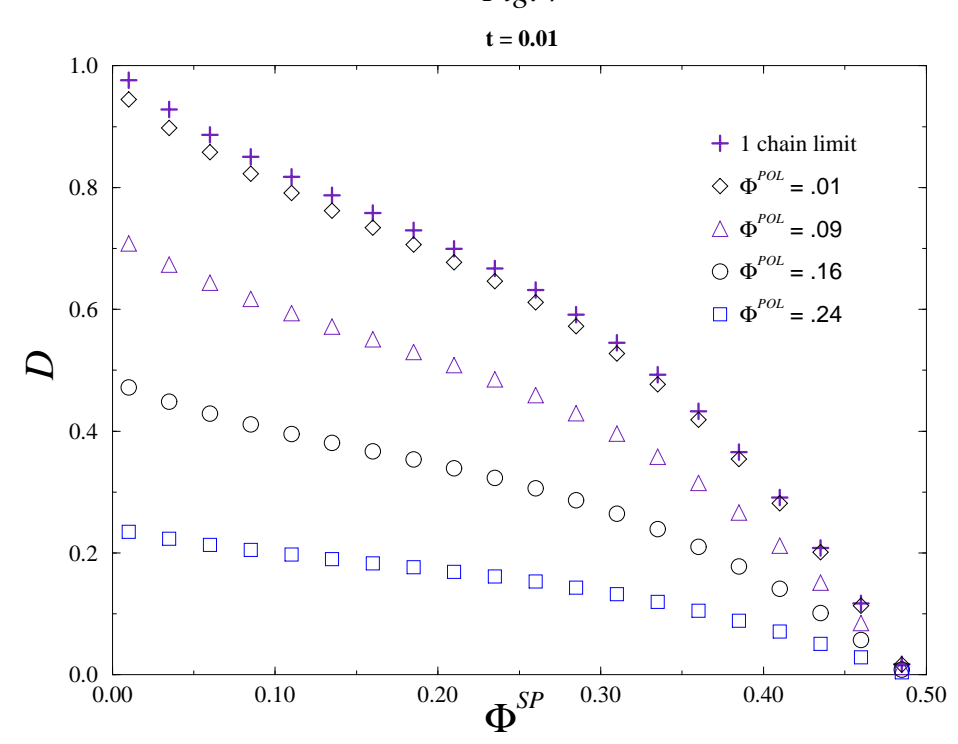

Fig. 9

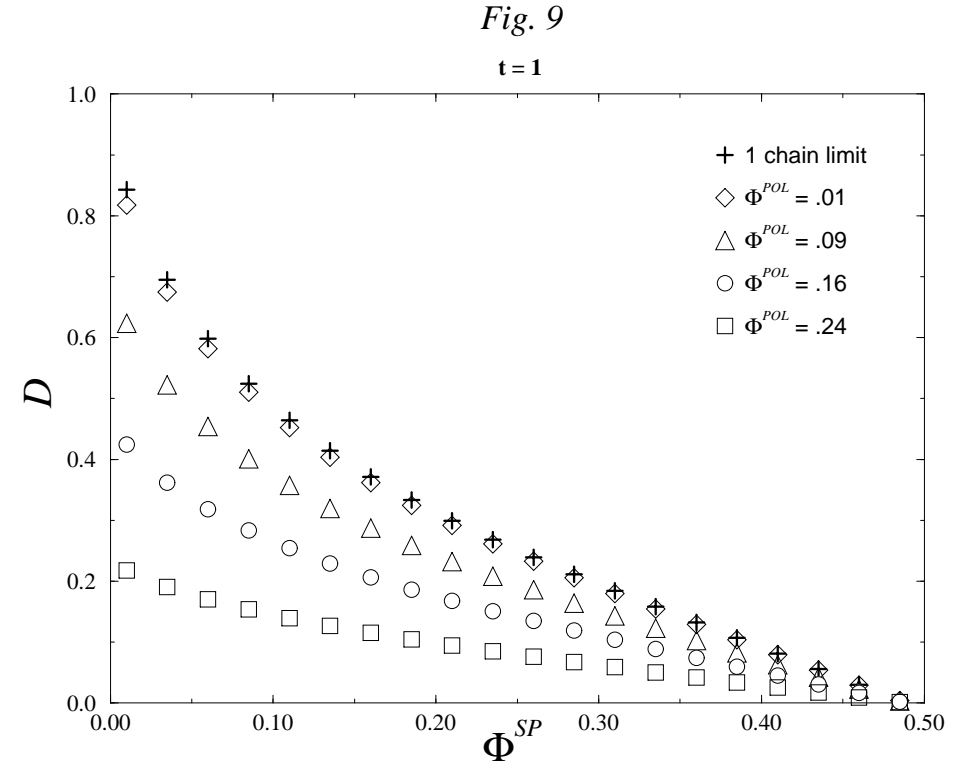


Fig. 10

$\mathbf{t}=10$

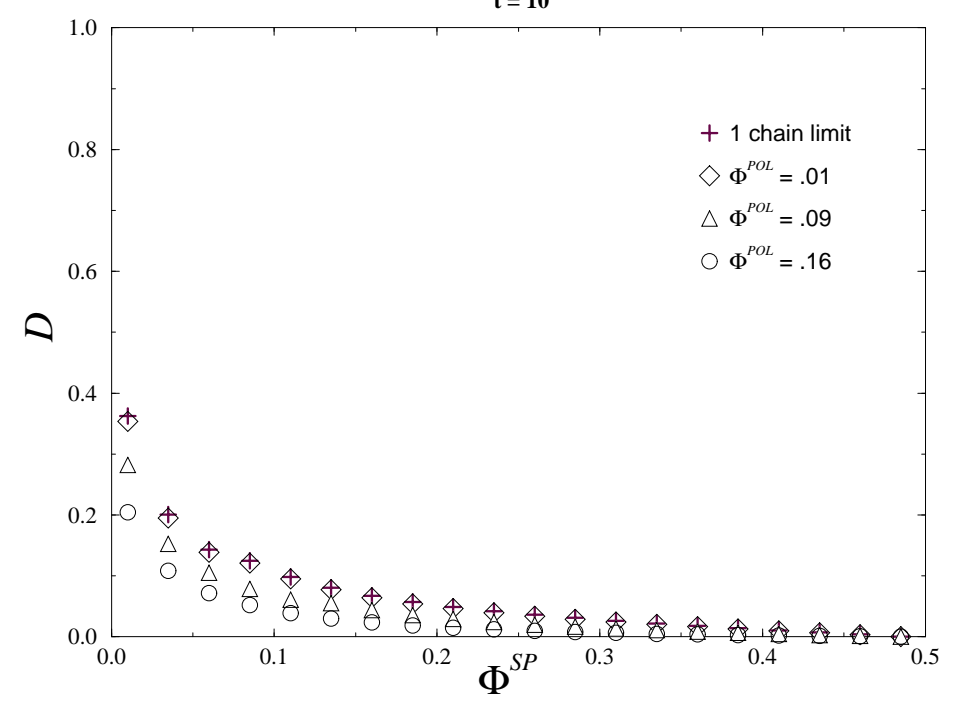

$\infty$

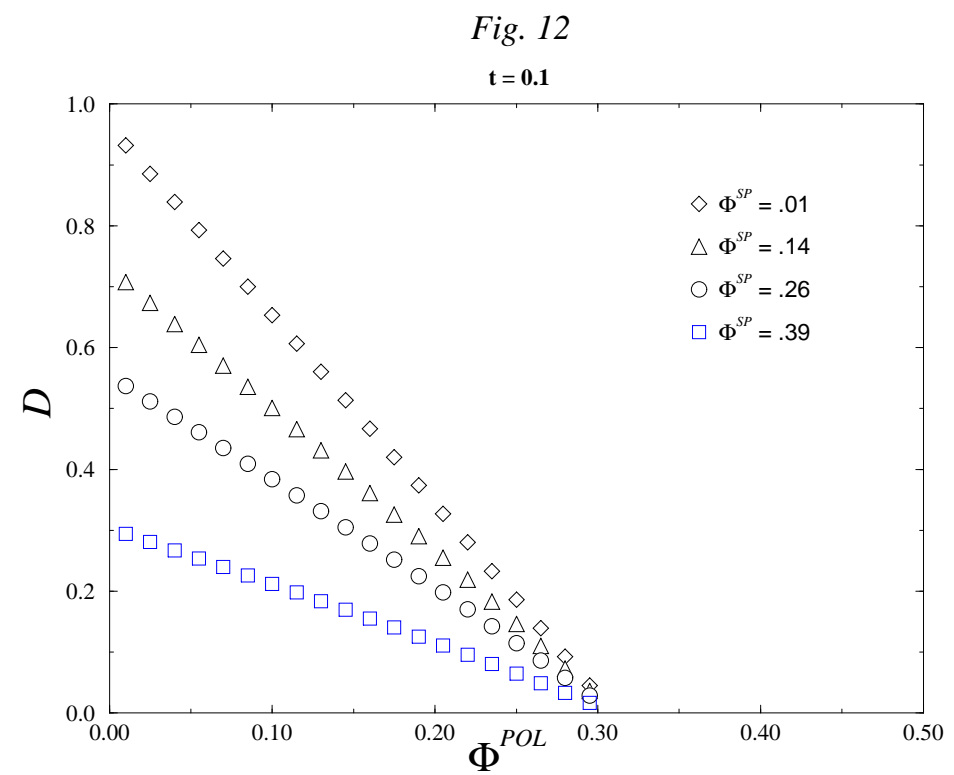

Fig. 11

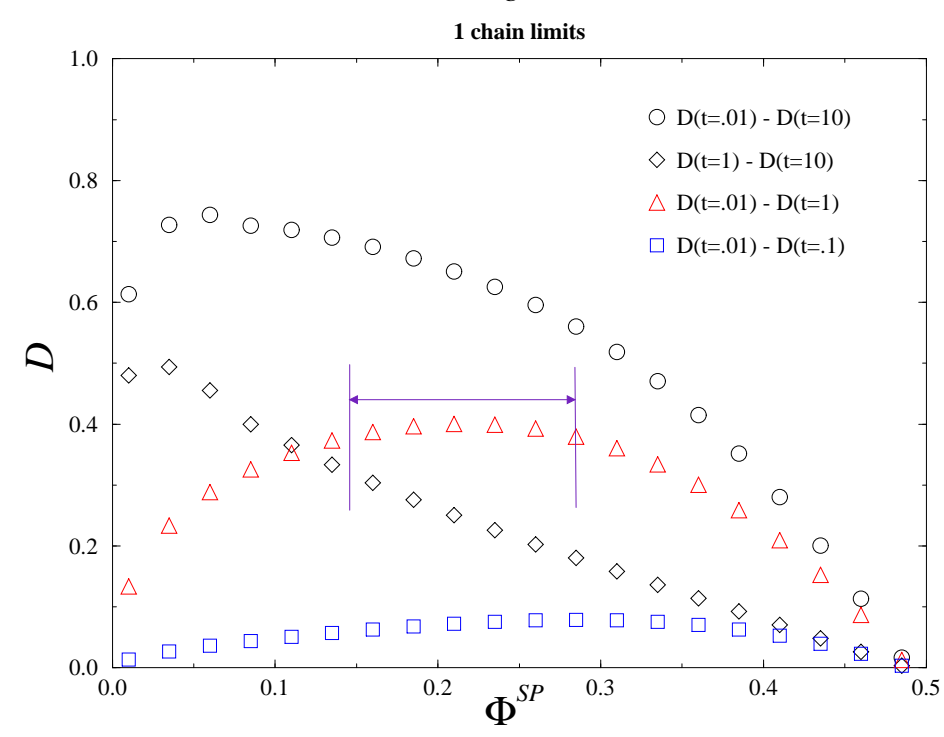

Fig. 13

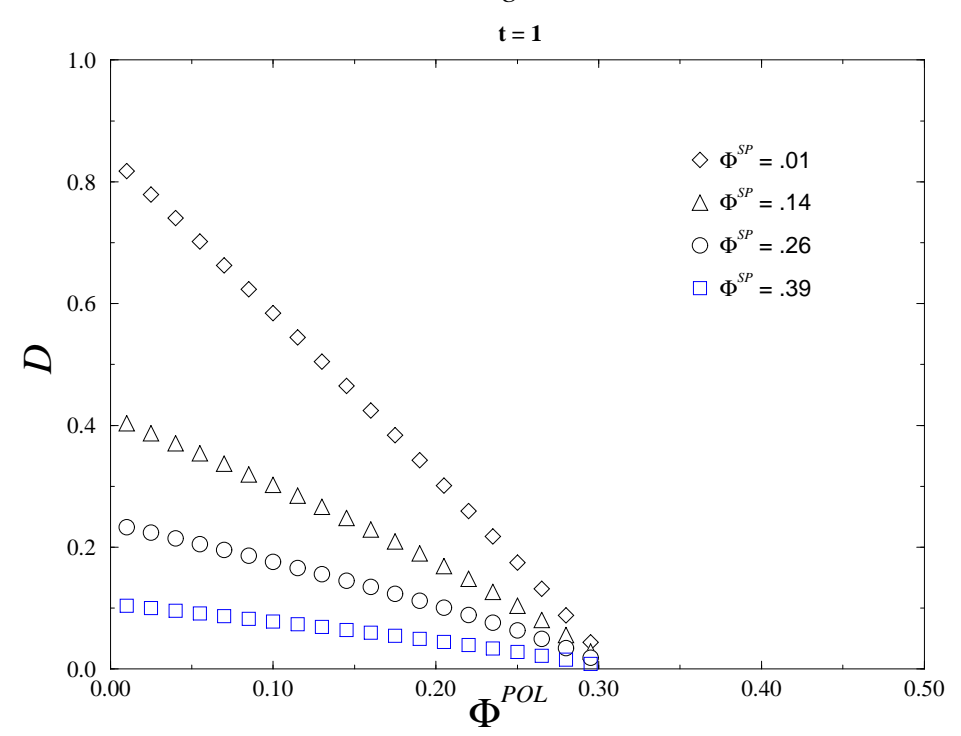


Fig. 14

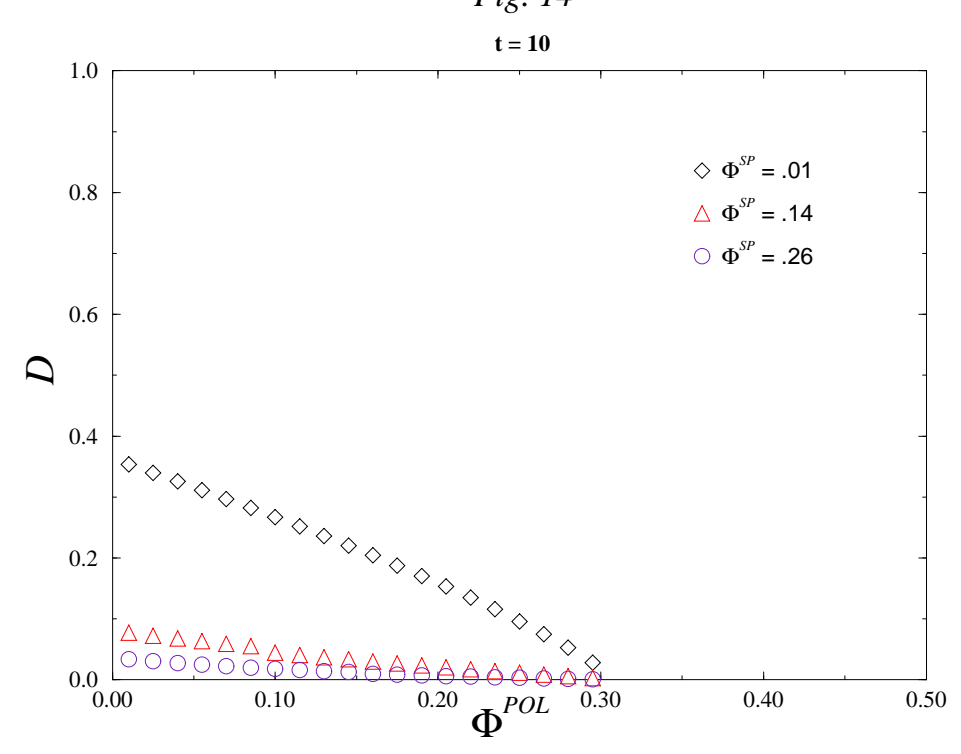

N

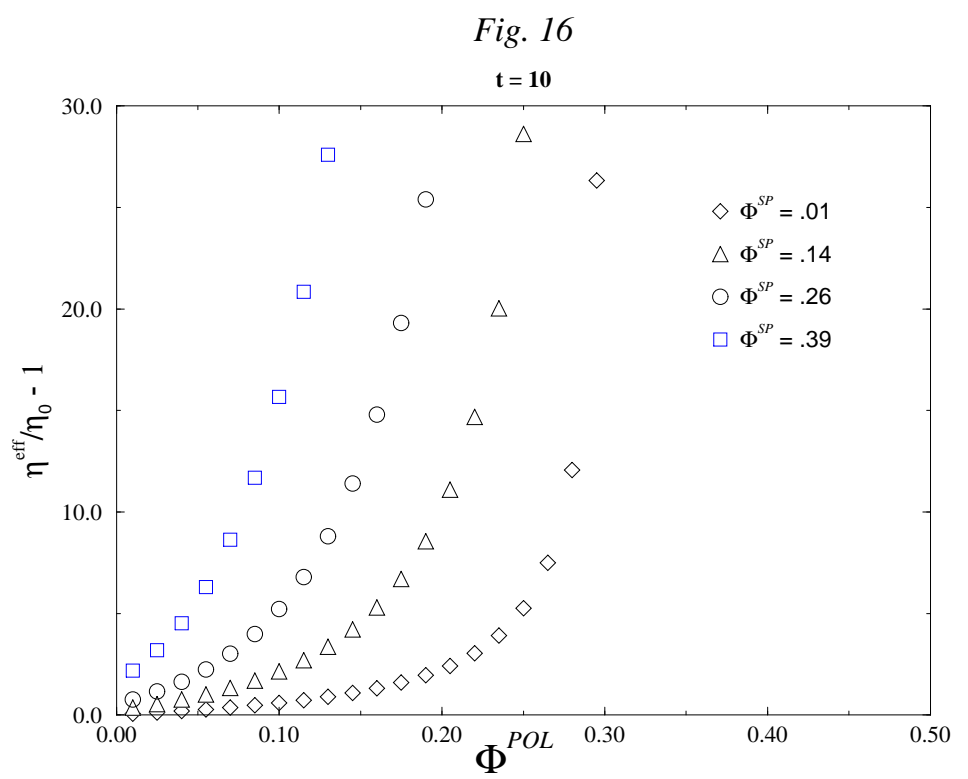

Fig. 15

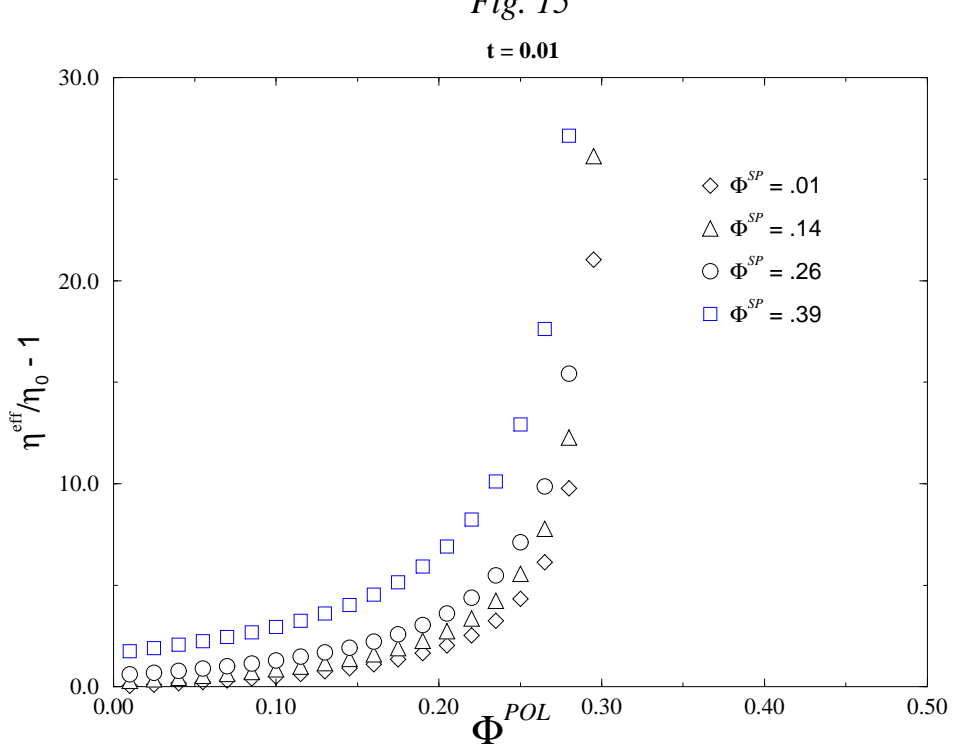

Fig. 17

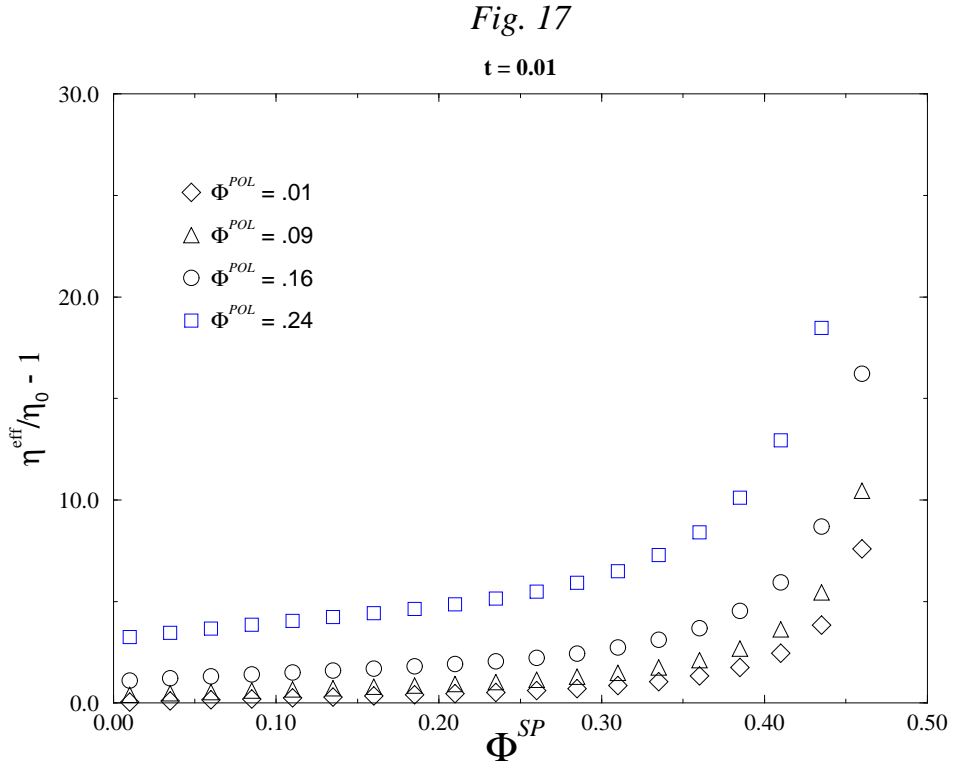




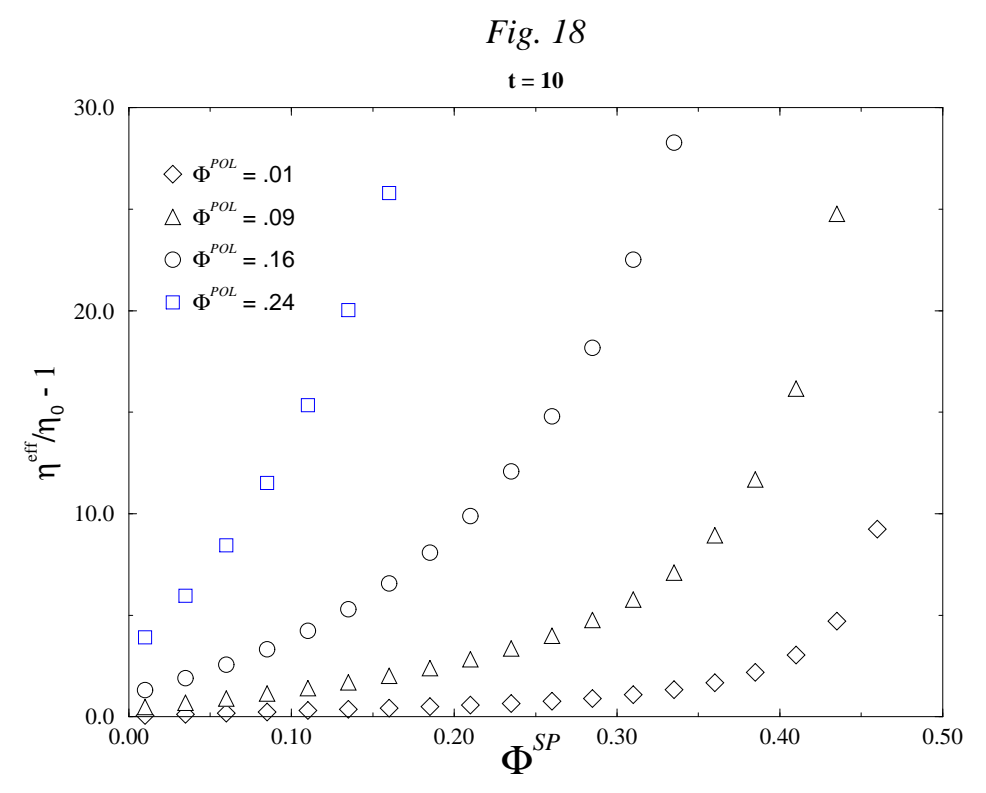

Fig. 20

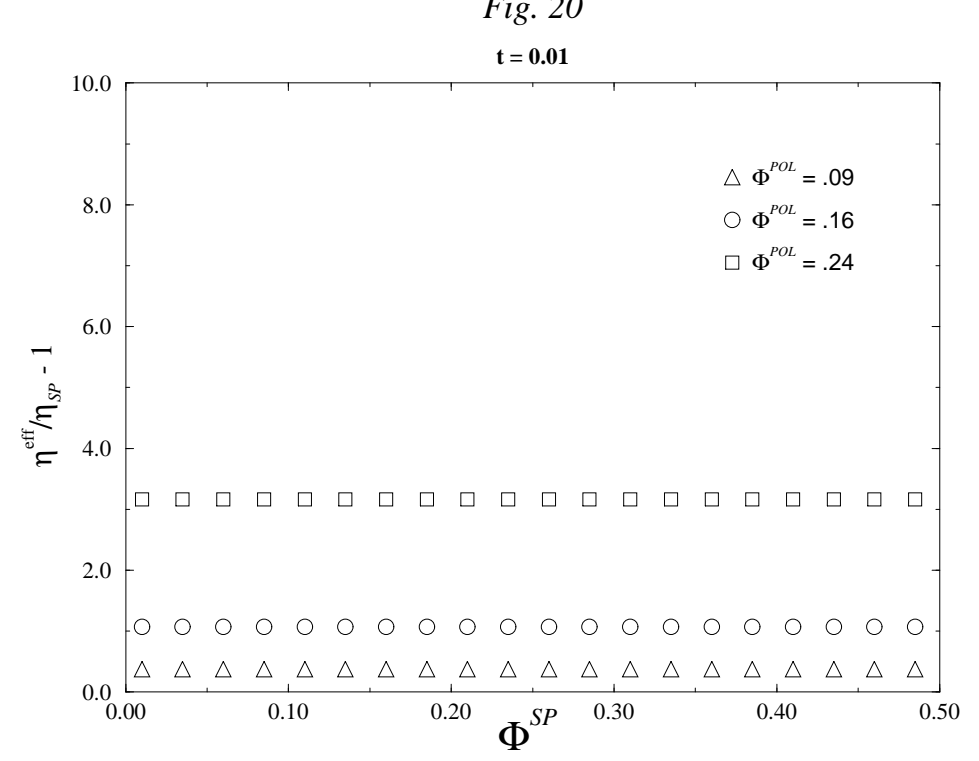

Fig. 19

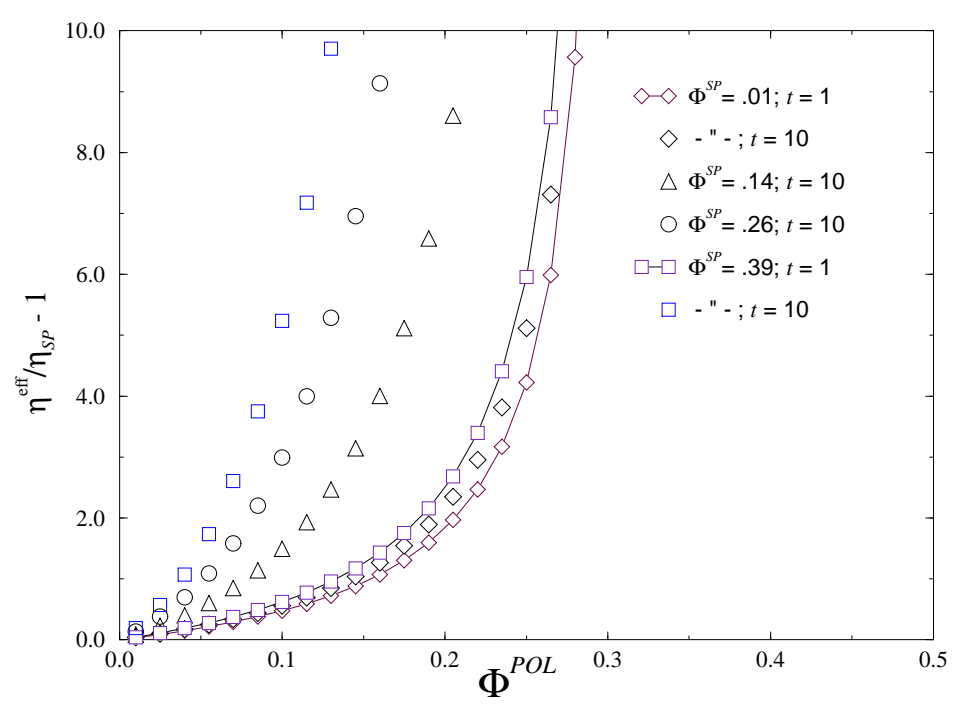

Fig. 21

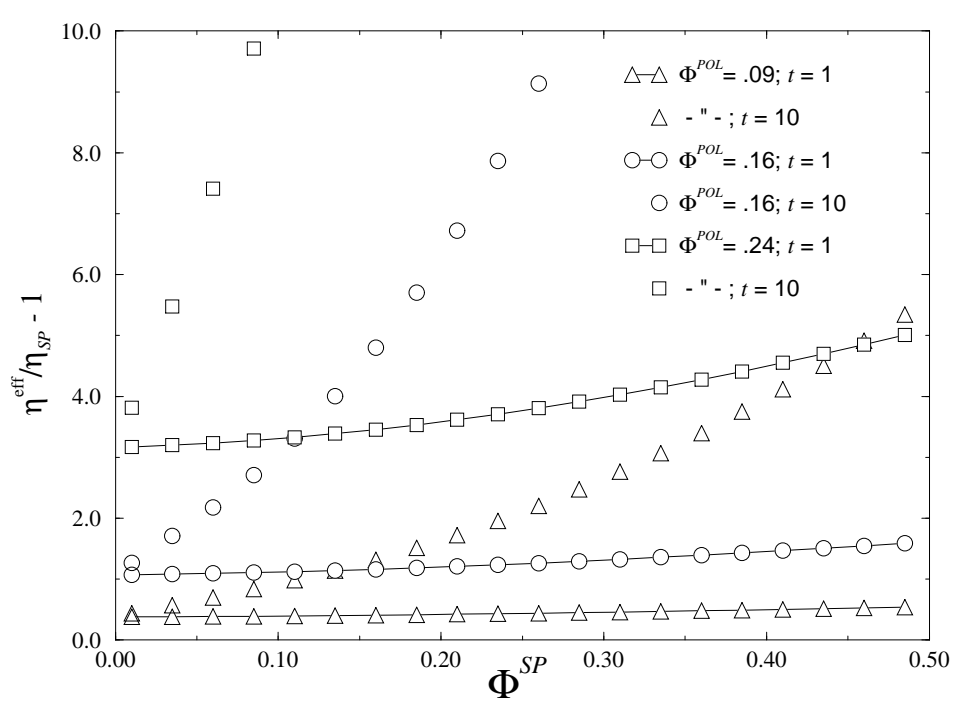

